\title{
Sex-specific regulation of metabolic health and vertebrate lifespan by AMP biosynthesis
}

Gwendoline Astre $^{1}$, Tehila Atlan ${ }^{1}$, Uri Goshtchevsky ${ }^{1}, K_{\text {Kobi Shapira }}^{2}$, Adi Oron-Gottesman ${ }^{1}$, Tomer Levy ${ }^{1}$, Ariel Velan ${ }^{1}$, Erez Y Levanon ${ }^{2}$, Joris Deelen ${ }^{3,4}$, and Itamar Harel ${ }^{1 *}$

${ }^{1}$ Department of Genetics, the Silberman Institute, the Hebrew University of Jerusalem, Givat Ram, Jerusalem, 91904 Israel.

${ }^{2}$ Mina and Everard Goodman Faculty of Life Sciences, Bar-Ilan University, Ramat-Gan, 52900, Israel. ${ }^{3}$ Max Planck Institute for Biology of Ageing, Cologne, Germany.

${ }^{4}$ Molecular Epidemiology, Department of Biomedical Data Sciences, Leiden University Medical

Center, Leiden, The Netherlands.

*Corresponding author (itamarh@mail.huji.ac.il)

\section{Abstract}

The loss of energy homeostasis seen during aging, is causally linked to multiple age-related pathologies. The AMP-activated protein kinase (AMPK) directly senses cellular energy levels, which are reflected in the ratio between AMP:ATP. However, the genetic regulation of vertebrate aging by the AMPK pathway remains poorly understood. Here, we manipulate ATP production by mutating $A P R T$, a key enzyme in AMP biosynthesis, and extend vertebrate lifespan in a male-specific manner. Using a multi-omics approach, we demonstrate that the $A P R T$ mutation restores metabolic plasticity, and identify a distinct transcriptional signature linking mitochondria with the sex-related differences in longevity. Accordingly, APRT mutant cells display a reduction in mitochondrial functions and ATP levels, and an increase in AMPK activity, resembling a persistent state of energy starvation. In-vivo, a fasting-like response was observed exclusively in male mutants, including resistance to a high-fat diet. Finally, intermittent fasting eliminated the longevity benefits mediated by the APRT mutation in males. Together, these data identify AMP biosynthesis as a sex-specific mediator of vertebrate longevity and metabolic health.

\section{Introduction}

Aging is associated with metabolic changes, including deregulated nutrient-sensing and mitochondrial dysfunction (Lopez-Otin et al., 2013). These changes are mechanistically linked to the onset of many age-related diseases such as diabetes, neurodegeneration, and cancer (Finkel, 2015; Niccoli and Partridge, 2012). Accordingly, a variety of longevity interventions (either dietary, genetic, or pharmacological), exert their effect by targeting primary metabolic pathways (Lopez-Otin et al., 2013; Smith et al., 2020). Surprisingly, many of these interventions induce a sex-specific response with respect to lifespan extension (Green et al., 
2021; Kane et al., 2018). However, the molecular mechanisms that mediate these differences are largely unknown (Austad and Fischer, 2016). Thus, a better understanding of how metabolic signals are relayed is crucial in designing tailored interventions to promote longevity.

In the context of vertebrate aging, a series of nutrient sensing pathways have been genetically investigated in mice. These involve the insulin and insulin-like growth factor-1 (IGF-1) signaling, as well as the downstream mechanistic target of rapamycin (mTOR) (Folgueras et al., 2018; Green et al., 2021). However, the genetic regulation of vertebrate aging by other metabolic pathways, specifically via the AMP-activated protein kinase (AMPK), remains poorly understood (Green et al., 2021; Lopez-Otin et al., 2013; Smith et al., 2020).

While the IGF-1 and mTOR pathways sense changes in glucose and amino acid levels, respectively, the AMPK pathway directly senses low energy in the cell, as reflected by a higher AMP:ATP ratio (Burkewitz et al., 2014; Hardie et al., 2012). In response, AMPK activation can restore the energy balance by inhibiting ATP-consuming processes, and activating ATPgenerating processes (Garcia and Shaw, 2017; Hardie et al., 2012). Therefore, AMPK is involved in many evolutionarily conserved aging-related interventions, such as dietary restriction, fasting, physical exercise, and metformin administration (Green et al., 2021; Smith et al., 2020). Indeed, genetic manipulation of the AMP:ATP ratio, or direct activation of AMPK have been shown to extend the lifespan of invertebrate models, including Drosophila (Stenesen et al., 2013; Ulgherait et al., 2014), C. elegans (Apfeld et al., 2004; Curtis et al., 2006; Greer et al., 2007; Mair et al., 2011), and yeast (Matecic et al., 2010). However, the conservation of similar genetic mechanisms in vertebrate aging remains unclear (Smith et al., 2020).

Genetic mouse models with constitutively active AMPK have so far demonstrated conflicting (and even detrimental) metabolic phenotypes (Carling, 2017; Woods et al., 2017; Yang et al., 2016; Yavari et al., 2016). These results may be due to the intricate tissue-specific composition of the vertebrate AMPK complex (Garcia and Shaw, 2017). Similarly, upstream to AMPK, genetic manipulation of AMP biosynthesis (the precursor for ATP) has been shown to extend Drosophila lifespan (Stenesen et al., 2013). However, the proposed mechanism in this case was thought to be invertebrate-specific (Camici et al., 2018), as it was predicted to actually decrease the AMP:ATP ratio. Thus, developing new genetic strategies are required to better understand how AMPK regulates vertebrate metabolism and longevity, either by tissue-specific activation of AMPK (Garcia et al., 2019), or by genetic manipulation of energy homeostasis. Notably, 
genetic dissection of vertebrate aging pathways using classical experimental models (namely the mouse and zebrafish), has been hindered by their relatively long lifespan.

The African turquoise killifish Nothobranchius furzeri has recently emerged as a powerful vertebrate model to investigate aging and related diseases (Astre et al., 2022; Baumgart et al., 2016; Cellerino et al., 2015; Harel and Brunet, 2015; Kim et al., 2016). These fish provide an attractive experimental platform as they possess a naturally compressed lifespan that is 6 or 10 times shorter than mice or zebrafish, respectively (Tacutu et al., 2018). They also exhibit typical vertebrate aging phenotypes, including a decline in cognitive functions and reduced fertility, as well as an increase in age-related pathologies (Astre et al., 2022; Cellerino et al., 2015; Harel and Brunet, 2015; Kim et al., 2016). Importantly, the killifish responds to conserved lifespan interventions, such as drug treatments and intermittent fasting (Terzibasi et al., 2009; Valenzano et al., 2006). The recently developed integrative genomic and CRISPRbased genome-editing platform has transformed the killifish into a bona fide genetic model that enables rapid exploration of vertebrate aging and diseases (Harel et al., 2015, 2016; Reichwald et al., 2015; Valenzano et al., 2015). However, genetic exploration of pro-longevity pathways has not been attempted so far.

Here we leverage the turquoise killifish as a powerful model to explore the genetic regulation of vertebrate aging by the energy-sensing AMPK pathway. In order to manipulate ATP levels, we generate a heterozygous mutation in the APRT gene, a key enzyme in AMP biosynthesis. AMP, the precursor of ADP and ATP, is formed by either the de-novo or salvage AMP biosynthetic pathways (Camici et al., 2018). The AMP salvage pathway is more energy efficient, and is predicted to be the primary source ( 90\%) of daily purine nucleotide biosynthesis across different organs (Johnson et al., 2019; L Ipata et al., 2011; Murray, 1971; Young et al., 2021). We then demonstrate that the APRT mutation extends the lifespan in a male-specific manner, and restores metabolic plasticity in old age (including glucose homeostasis). Interestingly, our findings are so far specific to APRT, as mutating another enzyme in the ATP synthesis pathway has no effect on the lifespan.

We have also identified a distinct gene expression signature that links mitochondrial functions with the sex differences in longevity, and have developed a cell-culture platform for killifish that allows us to explore the mechanisms involved. We use this system to show a reduction in mitochondrial functions and ATP levels, and an increase in AMPK activity in cells derived from $A P R T$ mutant males, to produce a phenotype that resembles a persistent state of starvation. 
These results are supported by the in-vivo findings of an increase in mitochondrial biogenesis and a fasting-like state exclusively in male mutants. Finally, intermittent fasting eliminates the longevity benefits mediated by the APRT mutation. Collectively, these data identify the AMP salvage pathway as a novel genetic regulator of vertebrate aging, and APRT as a promising pharmacological target for pro-longevity interventions.

\section{Results:}

\section{Generation of a genetic model for APRT, a member of the AMP biosynthesis pathway}

To genetically alter organismal energy homeostasis, we manipulated AMP biosynthesis (Figures 1, S1). For this purpose, we selected Adenine phosphoribosyltransferase (APRT), which catalyzes the formation of AMP from adenine and PRPP (5-phosphoribosyl-1pyrophosphate) as our primary target (Figure 1a). CRISPR-based genome editing (Astre et al., 2022; Harel et al., 2016), was used to generate an 8 base-pair deletion ( $\Delta 8)$ allele in the killifish $A P R T$ gene, which was predicted to give rise to a premature stop codon and a loss of function (Figure 1b). We then outcrossed this line for 8 generations to reduce the effect of potential offtarget mutations.

Mating heterozygous $A P R T^{\Delta 8 /+}$ pairs follows the expected Mendelian ratios with respect to the genotypes of the fertilized eggs ( $p=0.9, \chi^{2}$ test, Figure $\left.S 1 a\right)$, and there was no significant difference in either fecundity (Figure S1b) of the pairs, or the egg size produced (Figure S1c). However, after hatching, we failed to detect any adult $A P R T^{\Delta 8 / \Delta 8}$ fish, suggesting that the homozygous mutation causes embryonic lethality ( $\mathrm{p}=0.0015, \chi^{2}$ test, Figure $\left.S 1 \mathrm{a}\right)$. Notably, an $A P R T$ homozygous mutation is similarly embryonic lethal in Drosophila (Stenesen et al., 2013). In mammals, APRT deficiency is associated with severe phenotypes in mice (e.g. premature death after weaning (Engle et al., 1996)), and to an autosomal recessive renal failure in humans (Bollée et al., 2012; Edvardsson et al., 2013). In view of the recent findings that AMP biosynthesis can extend Drosophila lifespan, albeit by a possible invertebrate specific mechanism (Camici et al., 2018; Stenesen et al., 2013), we were curious to examine the effect of the APRT heterozygous mutation on vertebrate lifespan.

\section{Male-specific lifespan extension in $A P R T^{\Delta 8 /+}$ heterozygous fish}

Our results indicate that $A P R T^{\triangle 8 /+}$ heterozygous male fish live significantly longer (17\% increase, log-rank test, $p=0.021$, Figure 1c). Surprisingly, the female lifespan is not significantly affected (log-rank test, $\mathrm{p}=0.86$ ) (Figure 1d), and remains comparable to that of 
WT males (Figure 1e). In order to examine the specificity of our phenotype, we also mutated adenylate kinase 2 (AK2), a second enzyme in the ATP synthesis pathway that was previously demonstrated to extend the Drosophila lifespan (Stenesen et al., 2013). AK2, like other AK isozymes, catalyzes the reversible conversion of the AMP and ADP adenine nucleotides $(2 \mathrm{ADP}=\mathrm{ATP}+\mathrm{AMP})($ Camici et al., 2018) (Figures 1a, S1d). A 'knock-in” approach (Astre et al., 2022; Harel et al., 2016), was used to generate an $A K 2^{\Delta 8 /+}$ allele (Figure S1e).

Like the $A P R T$ mutation, the $A K 2^{48 / \Delta 8}$ homozygous mutation is embryonic lethal (Figure $\mathrm{S} 1 \mathrm{f}$ ), But, in contrast to the findings in Drosophila (Stenesen et al., 2013), no extension of the lifespan extension was observed in either male (log-rank test, $\mathrm{p}=0.87$ ) or female (log-rank test, $\mathrm{p}=0.7$ ) heterozygous fish (Figure $\mathrm{S} 1 \mathrm{~g}, \mathrm{~h}$ ). Taken together, our findings highlight the role of the AMP salvage pathway, and specifically of the APRT enzyme, in modulating vertebrate lifespan. Furthermore, our sex-specific data provide an exciting opportunity to explore the molecular mechanisms of sex differences in aging (Austad, 2006; Austad and Fischer, 2016; Horstman et al., 2012). To examine how the longevity benefits by the $A P R T^{48 /+}$ mutation are produced, we performed an extensive multi-omics analysis, initially focusing on male fish (Figure 1f)

\section{APRT heterozygous fish display an age-dependent signature of canonical AMPK targets}

Energy homeostasis and metabolic functions depend on organismal age and nutrient availability (Lopez-Otin et al., 2013). We therefore performed a transcriptomic analysis comparing the liver, a primary metabolic organ, from young (6.5 weeks) or old (15 weeks) male wild-type (WT) or $A P R T^{\Delta 8 /+}$ heterozygous fish. To evaluate the influence of food availability and metabolic plasticity, we compared the livers from fully fed or fasted (fasting for $24 \mathrm{~h}$ ) animals of each age/genotype (a total of 8 experimental groups, Figure 1f). This scheme was designed to examine whether nutrient sensing is indeed deregulated during killifish aging, and if so, to determine the effect of the $A P R T^{\Delta 8 /+}$ mutation.

A principal component analysis (PCA) for transcript levels revealed separation of samples depending on the experimental conditions (Figure 2a for PC1/3, and Figure S2a for PC1/2). For example, old $A P R T^{48 /+}$ heterozygous fish separated with young fish, suggesting a possible rejuvenated phenotype (Figure 2a, top). Age-dependent (Figure 2a, bottom) and genotypedependent (Figure S2a, left) patterns were also observed. The lack of available antibodies directed against the killifish APRT protein, requires the evaluations of APRT transcript levels. As expected, the levels of $A P R T$ transcripts were significantly reduced in heterozygous fish, 
probably as a result of nonsense-mediated mRNA decay (NMD) (Figure S2b). These findings further suggest that the APRT $\triangle 8$ is a loss-of-function allele.

In order to obtain an overview of hepatic aging in killifish, we first screened for genes differentially expressed between young and old control fish (WT, fully fed), and conducted gene set enrichment analysis (GSEA), using gene ontology (GO). The results revealed 1242 downregulated and 1509 upregulated genes, with an FDR of 0.05. Among the significantly enriched pathways, we could identify classical 'hallmarks of aging' signatures (López-Otín et al., 2013), including genes associated with genomic instability, mitochondrial dysfunction, deregulated nutrient sensing, and cell cycle (Figure 2b, Table S2). To identify a robust signature of aging, we then expanded this analysis to include all age-related changes, independent of genotype or feeding condition (Figures 2c, S2c). This comparison identified increases in inflammatory responses, or 'inflammaging' (Benayoun et al., 2019; Franceschi et al., 2018), as a robust age-related transcriptional signature.

Are old $A P R T^{\Delta 8 /+}$ fish truly rejuvenated, as predicted by the PCA? To identify differentially expressed genes that co-depend on both genotype and age, we performed interaction analysis, followed by GSEA using GO. Under both feeding conditions, we identified canonical AMPK downstream targets that are co-dependent on both age and genotype (Figure 2d, Table S2). These are involved in autophagy, energy metabolism (i.e. insulin and glucose homeostasis), cell-cycle-related pathways, and lipid metabolism.

\section{Age-related decline in nutrient sensing is prevented in old $A P R T^{\Delta 8 /+}$ fish}

To further explore energy metabolism, we performed metabolomic analysis on killifish livers (Figures 3, S3a), using the same experimental design performed for the transcriptomics (Figure 1f). For this purpose, we focused on polar metabolites, such as nucleic acids, sugars, and small organic acids that are typically involved in primary metabolism. Similar to the transcriptomic analysis (Figure 2), PCA demonstrated that under fully fed conditions, samples primarily segregated according to age (Figure 3a, top), while genotype dependency was revealed under fasting conditions (Figure 3a, bottom). These data confirm the importance of feeding regimens in exposing age- and genotype-specific metabolic signatures.

Out of a total of 160 unique metabolites, 140 passed our quality control tests (Table S3). We then applied hierarchical clustering to all 140 detected metabolites, and focused on a subgroup whose signature displays the following characteristics (Figure S3a): a) dependent on nutrient availability (i.e. food dependent); b) exhibits significant differences between groups; c) 
displays a restoration of youthful levels in old heterozygous fish under fasting conditions (see Methods for full statistical analysis). These selected metabolites are members of primary energy and biosynthetic pathways, including amino acid and carbohydrates metabolism, glycolysis, nucleoside and nucleotide metabolism, and the pentose phosphate pathway (PPP) (Figure 3b,c).

For example, the levels of members of the PPP were globally reduced in the livers of young and old APRT heterozygous fish. Limiting the PPP is an integral part of the mammalian response to fasting (Adams et al., 1983; Jenniskens et al., 2002; Lee et al., 2019; Meister, 1988), and inhibition of the PPP was recently shown to promote longevity in C. elegans by mimicking dietary restriction (Bennett et al., 2017). Overlaying the levels of individual metabolites onto the glycolysis and ATP salvage pathways, demonstrated a similar behavior (Figure 3c,d), with only a handful of intriguing outliers (e.g. GMP and IMP). Taken together, our metabolomic analysis suggests that old fish suffer from deregulated nutrient sensing, which is partially rejuvenated in old $A P R T^{48 /+}$ heterozygous fish.

The genotype-age interactions also predicted altered lipid metabolism (Figure 2d), which has been recently implicated in aging and longevity in both mammals (Johnson and Stolzing, 2019; Palavicini and Han, 2021) and fish (Ahuja et al., 2019; Milinkovitch et al., 2018). This prompted the question as to which lipid species are linked to the metabolic rejuvenation observed in old $A P R T^{\Delta 8 /+}$ fish. Like the metabolomic signatures, the PCA demonstrated agedependent differences under fully-fed conditions (Figure S3b, top), with a genotype contribution when the animals were fasted (Figure S3b, bottom). Applying hierarchical clustering, revealed a complex signature that is affected by age, genotype, and feeding conditions (Figure S3c, left). We then scanned our data for lipid species that exhibit restoration of youthful patterns in old $A P R T^{\Delta 8 /+}$ heterozygous fish under fasting conditions. The results highlighted a distinct group of glycerophospholipids, including lysophosphatidylcholine (LPC), lysophosphatidylethanolamine (LPE), and phosphatidylinositols (PI) (Figure S3c,d). Interestingly, while the level of LPEs increased with age during fasting in WT animals, the heterozygous old fish retained the lower levels characteristic of youth.

In this context, low levels of plasma LPE (i.e. LPE(P-16:0)) were recently associated with exceptional human longevity (Pradas et al., 2019), and LPC levels have been implicated in human aging and mitochondrial functions (Semba et al., 2019). It may also be pertinent that although PIs constitute only a small portion of cellular phospholipids, they have recently 
emerged as key modulators of energy metabolism, including insulin sensitivity, and obesity (Balla, 2013; Bridges and Saltiel, 2015; Lundquist et al., 2018). Taken together, our findings highlight a role for the AMP salvage pathway, and specifically for the APRT enzyme, in modulating vertebrate lifespan and restoring metabolic plasticity in males.

\section{Genotype-age interaction analysis identifies a male-specific mitochondrial signature}

What mediates the observed sex differences? In order to address this question we performed transcriptomic analysis on $A P R T^{48 /+}$ heterozygous females under metabolic bassline (experimental groups \#9-12, Figure 4a, S4a). In agreement with the findings in males, APRT transcripts were significantly reduced in heterozygous fish (Figures S2b, S4b). GSEA for agerelated differential gene expression confirmed that 'inflammaging' is a conserved aging hallmark in both sexes (Figure 4b, Table S2). However, there were distinct sex-linked biases observed, specifically with respect to carbohydrate and lipid metabolism (Figure 4b,c).

Interestingly, some pathways demonstrated an additional genotype dependency, such as predicting an age-dependent inhibition of 'Mitochondrial respiratory chain complex I assembly' in $A P R T^{\Delta 8 /+}$ heterozygous males (Figure $4 \mathrm{~b}$ ). Since, we anticipated that pathways modified specifically in male $A P R T^{\Delta 8 /+}$ fish (and not in females) during aging, are more likely to make a functional contribution to the longevity effect, we performed a similar genotype-age interaction analysis on the female transcriptomic data (Figure 2d). Comparing our findings between sexes indeed highlighted male-specific mitochondrial-related pathways (Figure 4d, Table S2). However, as the interaction analysis considers both genotype and age, the ways in which the largely opposite responses between sexes are reflected in gene expression are nonintuitive. We therefore calculated the gene expression fold change between heterozygous and WT fish for each experimental condition, which provided a simple representation of the sexbiased transcriptional response (Figure $\mathrm{S} 4 \mathrm{c}$ ).

These results of this analysis predict that mitochondrial dynamics, such as copy numbers and function, could be co-dependent on age, sex, and genotype. Among the core regulators of mitochondrial dynamics, genes responsible for mitochondrial biogenesis, and specifically $P G C$-1 $\alpha$ (Peroxisome proliferator-activated receptor gamma coactivator 1-alpha), which is a master regulator of mitochondrial biogenesis, displayed a strong sex-dependency when compared to fission or fusion (Figure S4d). This phenotype is consistent with AMPK activation, as $P G C-1 \alpha$, is an AMPK target (Garcia and Shaw, 2017) (Figure S4d). Accordingly, a significant increase in mitochondrial DNA (mtDNA) copy number was observed, specifically 
in the livers of old male $A P R T^{\Delta 8 /+}$ fish (Figures 5a). Similar patterns were observed in other organs, such as the tail, suggesting a systemic response (Figure $5 b$ ).

Interestingly, genotype-age interactions that are shared between males (both types of feeding conditions) and females, highlighted four pathways involving RNA modification, where such modifications, including RNA-editing (Figure S5a) (Eisenberg and Levanon, 2018), are known to be tightly linked to mitochondrial dynamics (Dhir et al., 2018; Sharma et al., 2019; Wiatrek et al., 2019). An in depth examination of A-to-I editing in our dataset identified an agedependent increase in RNA-editing in males, and a decrease in females (Figure S5b-d). Although age-dependent RNA-editing was previously predicted (Porath et al., 2017), our ability to identify this phenomenon in killifish was made possible by the low polymorphic background (Valenzano et al., 2015), and by developing a new computational editing detection strategy (see Methods section).

These data highlight pathways that display significant sex differences during aging, and a subset that is further altered in response to the $A P R T^{\Delta 8 /+}$ heterozygous mutation (particularly related to energy metabolism and mitochondrial physiology). However, evaluating whether these pathways contribute to the observed differences in lifespan, requires a cellular platform in order to explore how mitochondrial functions are altered by the $A P R T^{\Delta 8 /+}$ heterozygous mutation.

\section{$A P R T^{\Delta 8 /+}$ cells have reduced mitochondrial functions that can be partially rescued by adenine supplementation}

To explore mitochondrial function on a cellular level, we developed primary fibroblast cultures derived from the tail fin of male WT and $A P R T^{\Delta 8 /+}$ fish (Figure 5c). Optimizing previously published protocols (Graf et al., 2013), allowed us to produce robust early-passage cultures, and use individual fish as biological replicates (without pooling). As expected, APRT $T^{\triangle 8 /{ }_{-}}$ derived cells display reduced proliferation when compared to WT cells (Figure 5d) and are partially resistant to 8 -azaadenine treatment (Figure 5e). This partial resistance is a consequence of the reduced ability to metabolize adenine and therefore to produce the toxic intermediate generated by 8-azaadenine (Jones and Sargent, 1974; Sahota et al., 1987). Together, these experiments demonstrate that killifish primary cells can serve as a robust platform for cell biology.

As the next stage, we used the seahorse platform to characterize mitochondrial functions ('Mito Stress' assay, Figure 6a). The results indicated that mitochondrial respiration (including basal 
respiration and ATP production), were significantly reduced in primary cells from $A P R T^{\Delta 8 /+}$ fish (Figure 6b-d). Similarly, there was a significant reduction in glycolytic functions, including glycolytic capacity and glycolysis, in heterozygous cells (Figure S6a-c). In contrast, mitochondrial functions, and specifically the level of ATP production, were unaltered in $A K 2^{48 /+}$-derived cells (Figure S6d). These data are in agreement with the comparable lifespan between WT and $A K 2$ heterozygous fish (Figure S1g,h). We next hypothesized that the observed phenotypes could be rescued by exogenously manipulating the nucleotide pools. APRT catalyzes the formation of AMP by using adenine as a precursor. Accordingly, our results revealed that adenine supplementation partially restores mitochondrial functions in heterozygous cells by increasing respiration (Figure 6c-d). These results provide support for the notion that the observed mitochondrial phenotypes are a direct consequence of APRT haploinsufficiency.

Could the observed phenotypes be attributed simply to changes in mitochondrial numbers, as seen in-vivo (Figures 5a)? To test this, we investigated mitochondrial morphology in the cultured cells. We observed that although mitochondrial branch length is slightly increased in heterozygous cells, the mitochondrial footprint remains essentially the same regardless of genotype (Figure 6e). These findings were further supported by direct measurement of mtDNA copy numbers in cells from male animals, which revealed comparable counts in WT and heterozygous cells (Figure S6e). Similar results were obtained in female-derived cells, pointing towards a cell autonomous mechanism. Importantly, the APRT-dependent effect on mitochondrial respiration was conserved in female-derived cells (Figure S6f), suggesting that the observed sex-biased phenotypes could be mediated by sex hormones (Mohapatra et al., 2020). However, exposure of fish to estradiol (E2) did not have a significant effect on mitochondrial copy number (Figure S6g).

Together, our findings demonstrate that the observed genotype- and sex-dependent mitochondrial biogenesis depend on yet unknown in-vivo signals, which are uncoupled from mitochondrial functions. As pharmacological or genetic inhibition of mitochondrial functions is a conserved longevity intervention (Baumgart et al., 2016; Lee et al., 2003; Sun et al., 2016; Tavallaie et al., 2020; Wang and Hekimi, 2015), our findings could provide a possible prolongevity mechanism downstream to APRT and AMPK. However, the question remains as to which pathways are directly involved in the male-specific increased longevity. 


\section{Increased AMPK activity and altered nucleotide ratios in APRT mutant cells produces a fasting-like state}

The data so far suggest that $A P R T^{\Delta 8 /+}$-derived cells experience altered energy metabolism. To further explore whether this condition resembles fasting on the protein level, we validated a panel of specific antibodies directed against key members of the cellular fasting response (Figure S7a,b). These were used to assess the phosphorylation of classical AMPK targets in WT and $A P R T^{\Delta 8 /+}$-derived cells, under either basal conditions, or low serum (serum starvation, Figure 7a). The results indicated that even under basal conditions, $A P R T^{\Delta 8 /+}$-derived cells display a significant activation of the AMPK pathway, which resembles the activation observed in WT cells under serum starvation. This increase is further heightened under serum starvation conditions, suggesting that the $A P R T^{\Delta 8 /+}$ mutation can sensitize cells to nutrient levels and induce a fasting-like state even under basal conditions. A genotype-dependent response to fasting was also observed in-vivo (evaluated by the inhibition of the mTOR signaling pathway, Figure S7c).

Mutating APRT, which is part of the adenosine nucleotide synthesis pathway, might be expected to alter the ratios of AMP, ADP, and ATP. Importantly, data in Drosophila (Stenesen et al., 2013) and worms (Apfeld et al., 2004; Greer et al., 2007) suggests that the ratios of these products (e.g., AMP:ATP) are altered in response to caloric restriction and that such alterations could be predictive of the organismal lifespan. It was therefore of interest to explore whether this effect is also conserved in vertebrates. For this purpose, we analyzed the primary cultures by liquid chromatography mass spectrometry (LC-MS). The results (Figures 7b) revealed that ATP levels are significantly decreased in $A P R T^{48 /+}$-derived cells $(\mathrm{p}=0.05$, $\mathrm{t}$-test), while the AMP levels seem to be unchanged, and there was a non-significant trend to lower ADP ratios ( $\mathrm{p}=0.14$, t-test). Overall, these data imply that the $A P R T^{\Delta 8 /+}$-derived cells exist in a low energy state. We were next curious to test whether a similar fasting-like state also occurs in the male $A P R T^{48 /+}$ heterozygous fish.

\section{A sex-specific fasting-like state is observed in male $A P R T^{\Delta 8 /+}$ fish}

A variety of physical traits have been linked to longevity interventions that exert their effect by manipulating organismal metabolism (Fontana et al., 2010; Lopez-Otin et al., 2013; Smith et al., 2020). These include reduced body size and fecundity, delayed maturity, and resistance to a high-fat diet. Although $A P R T^{\Delta 8 /+}$ mating pairs exhibited no significant differences in fecundity (Figure S1b), male heterozygous fish were slightly smaller than their WT 
counterparts (Figure S7d) and experienced delayed maturity (Figure S7e). In contrast, the growth of female fish was unaffected by the $A P R T^{\Delta 8 /+}$ mutation (Figure S7d). As the next step, we developed a short-term high-fat diet regimen, which efficiently increases the amount of hepatic lipid droplets (specifically in old fish, Figure S7f). Applying this protocol to both male and female fish revealed that old $A P R T^{\Delta 8 /+}$ males are completely resistant to high-fat diet, and maintained hepatic lipid levels comparable to those of untreated control fish (Figure 7c).

\section{Intermittent fasting eliminates the longevity benefits mediated by the $A P R T$ mutation}

Our data demonstrate that the $A P R T^{\Delta 8 /+}$ mutation induces a fasting-like physiological state, specifically in male fish. This has led us to hypothesize that the $A P R T^{\Delta 8 /+}$-mediated longevity benefits might be effective only under normal feeding conditions, and may be negated by fasting. Excitingly, although the median lifespan of both male and female fish was significantly extended by life-long intermittent fasting, we could not detect any further extension in $A P R T^{48 /+}$ male fish (log-rank test, $\mathrm{p}=0.3$, Figure $7 \mathrm{~d}$ ). This observation suggests that the longevity effect achieved by intermittent fasting were dominant over the APRT-dependent sex differences. It is important to note that the effects of dietary restriction are themselves thought to be partially mediated by sex-specific molecular responses (Figures $4 \mathrm{~d}$ and S4c) (Kane et al., 2018) (see Discussion).

Together, our findings demonstrate that mutating APRT, a member of the AMP biosynthesis pathway, induces a sex-specific longevity effect. This effect is mediated by restoring metabolic homeostasis, modulating mitochondrial functions, and inducing a fasting-like state that involves distinct AMPK downstream regulators. In conclusion, our findings suggest that the AMP-salvage pathway and AMPK could function as a rheostat for vertebrate healthspan and longevity (Figure 7e).

\section{Discussion:}

Various aspects of energy balance and metabolism are known to be differently regulated between males and females of many species (Link and Reue, 2017; Mauvais-Jarvis et al., 2017; Wu and O'Sullivan, 2011). Such differences are evolutionarily linked to the unique metabolic requirements and energy partitioning required during reproductive life (Austad, 2011; Mauvais-Jarvis, 2015; Naqvi et al., 2019). With age, these characteristics also contribute to the sex-biased predisposition to various diseases, such as Alzheimer's disease and type 2 diabetes (Hägg and Jylhävä, 2021; Tramunt et al., 2020). 
Along these same lines, sexual dimorphism has been detected in many metabolic pathways including those involving growth hormone (Sun et al., 2017), mTOR (Baar et al., 2016), sirtuins (Sharples et al., 2015), and IGF-1 (Ashpole et al., 2017), which are associated with longevity and dietary restriction(Kane et al., 2018). Accordingly, genetic or pharmacological manipulation of these pathways in mice often confers sex-specific longevity benefits (Green et al., 2021). For example, administration of metformin, which interacts with mitochondrial complex I (and is a non-specific AMPK activator) (Pernicova and Korbonits, 2014), produces trending male-specific longevity bias as seen in the Intervention Testing Program (ITP, https://phenome.jax.org/projects/ITP1). However, the molecular mechanisms behind these longevity differences are largely unknown (Austad, 2011; Austad and Fischer, 2016), and could provide exciting opportunities for manipulating health and disease.

By exploring the basis for the sex-dependent longevity of the APRT mutants, we identified mitochondrial functions as a possible molecular mechanism. Accordingly, mitochondrial DNA copy numbers are increased, specifically in old $A P R T$ heterozygous males. In addition, the results of cellular assays revealed that heterozygous cells exhibit a reduction in mitochondrial functions and ATP levels, coupled with an increase in AMPK activity, a combination which resembles a persistent energy starvation. Mitochondria function and copy number are known to exhibit an evolutionarily-conserved sex bias (Ballard et al., 2007; Borras et al., 2007; Di Florio et al., 2020; Justo et al., 2005; Klinge, 2008; Kristensen et al., 2019; Valle et al., 2007; Ventura-Clapier et al., 2017). For example, the higher mitochondrial DNA copy number seen in females (Figures 5a) could suggest that this pathway is naturally optimized in females (potentially due to the high metabolic demand of egg production). As a result, an increase in mitochondria numbers by the APRT mutation, is more likely to be apparent in males (Justo et al., 2005; Valle et al., 2007).

Insight into how metabolic signals are integrated during aging in males and females opens up new possibilities for the design of tailored interventions that promote healthy aging. However, a mechanistic understanding of how these signals are transmitted to the tissues, cells, and organelles is still lacking. Our findings uncouple between the signals that regulate mitochondrial function to the ones that promote biogenesis. Specifically, the mitochondrial functions observed here are regulated in a cell-autonomous manner, and are genotype dependent regardless of whether the cells concerned were isolated from male or female fish (Figures 6, S6, S7). In contrast, mitochondrial DNA copy numbers appear to be dependent on physiological signals, as the in-vivo phenotypes were not present in primary cells. Although 
recent studies suggest sex hormones as a possible mediator (Besse-Patin et al., 2017; Hamilton et al., 2016; Zhou et al., 2020), estrogen exposure alone was not sufficient to replicate our findings (Figures S6g). Thus, exploring these signals in future studies could provide a powerful tool for systemic alteration of mitochondrial biogenesis in-vivo.

The longevity effects of intermittent fasting were dominant over the APRT-dependent sex differences, although this must be reconciled with the observation that intermittent fasting affects both sexes, while APRT has an impact only on male fish. Increasing evidence from rodents suggests that the beneficial effects of dietary restriction are partially mediated by sexspecific regulators (Figures 4d and S4c), and see (Green et al., 2021; Kane et al., 2018). Notably, in genetic mouse models of APRT deficiency, males similarly display increased susceptibility (Evan et al., 2001), suggesting that the sex differences in AMP metabolism could be evolutionarily conserved (Naqvi et al., 2019).

The observation that manipulating AMP biosynthesis by mutating APRT (and not AK2), restores metabolic plasticity in old age, and extends the lifespan in a sex-specific manner, raises the possibility that designing a pharmacological inhibitor for APRT could be an attractive prolongevity intervention. However, although significant efforts have been invested in Leishmania research (Boitz et al., 2012), a selective APRT inhibitor is still missing. Intriguingly, APRT inhibition is predicted to actually reduce AMP levels and decrease AMP:ATP ratios. Therefore, the observed AMPK activation is counterintuitive under steady-state conditions, as seen in Drosophila (Camici et al., 2018; Stenesen et al., 2013). However, this phenomenon could simply suggest that under low AMP production rates, the cell is more sensitive to spikes in energy consumption (e.g. cell division), or there is an as yet unidentified regulatory mechanism for AMPK (Yee et al., 2014).

Using the turquoise killifish model, we identify that AMP biosynthesis is a sex-specific regulator of metabolic health and vertebrate lifespan. However, to what extent are our findings evolutionarily conserved? A recent study (Lin et al., 2021) identified longevity-associated rare coding variants that converged on a number of pathways, including the AMPK signaling pathway. Thus, our findings suggest that the AMP biosynthesis and AMPK could function as an evolutionary conserved pathway that regulates vertebrate healthspan, and longevity. 


\section{Acknowledgements}

We thank the Harel lab, Sagiv Shifman, Anne Brunet, and Berenice Benayoun for stimulating discussion and feedback on the manuscript. We thank Ella Yanay and Ashayma Abu-tair for help with killifish maintenance, Yaakov Nahmias and Konstantinos Ionnidis for help with the Seahorse assay, Naomi Melamed-Book and Rachel Rosen from the core facilities (HUJI), Ifat Abramovich, Eyal Gottlieb, Sergey Malitsky and Maxim Itkin for help with mass spectrometry, and Param Priya Singh and Anne Brunet for advice with the GSEA code. Supported by the Zuckerman Program (I.H.), Abisch-Frenkel Foundation 19/HU04 (I.H.), ISF 2178/19 (I.H.), Israel Ministry of Science 3-17631 (I.H.), 3-16872 (I.H.), the Moore Foundation GBMF9341 (I.H.), BSF-NSF 2020611 (I.H.), the Israel Ministry of Agriculture 12-16-0010 (I.H), and the Lady Davis Postdoctoral Fellowship (G.A).

\section{RESOURCE AVAILABILITY}

\section{Lead Contact}

Further information and requests for resources and reagents should be directed to and will be fulfilled by the Lead Contact, Itamar Harel (itamarh@mail.huji.ac.il).

\section{Data and Code availability}

All raw RNA sequencing data, as well as processed datasets could be found in the GEO database, accession number GSE190757. All Lipidomic and metabolomic datasets could be found in Supplementary Table S3. The codes and results supporting the current study are available in the GitHub repository for this paper https://github.com/Harel-lab/APRT-sexdifferences.

\section{EXPERIMENTAL MODEL AND SUBJECT DETAILS}

\section{African turquoise killifish strain, husbandry, and maintenance}

The African turquoise killifish (GRZ strain) were housed as previously described (Astre et al., 2022; Harel et al., 2016). Fish were housed at $28^{\circ} \mathrm{C}$ in a central filtration recirculating system with a $12 \mathrm{hr}$ light/dark cycle at the Hebrew University of Jerusalem (Aquazone ltd, Israel). Until the age of 2 weeks, fish were exclusively fed with live Artemia (\#109448, Primo). Starting week 3, fish were fed three times a day on weekdays (and once a day on weekends), with GEMMA Micro 300 Fish Diet (Skretting Zebrafish, USA), supplemented with Artemia twice a day. In these conditions, killifish lifespan was approximately 4-6 months. Both the APRT and AK2 loss-of-function alleles were maintained as heterozygous and propagated by crossing 
with wild-type fish. All turquoise killifish care and uses were approved by the Subcommittee on Research Animal Care at the Hebrew University of Jerusalem (IACUC protocol \#NS-1815397-2).

\section{CRISPR/Cas9 target prediction and gRNA synthesis}

CRISPR/Cas9 genome-editing protocols were performed according to (Astre et al., 2022; Harel et al., 2016). In brief, for targeting APRT and AK2, conserved regions that were upstream of functional or active protein domains were selected. gRNA target sites were identified using CHOPCHOP (https://chopchop.rc.fas.harvard.edu/) (Labun et al., 2019), and were as follows (PAM sites are in bold): APRT Exon 3: 5'- GGGGTCCAAACAGGAAGCCACGG-3'; AK2 Exon 2: 5'- GGCCACCGGAGACATGCTGAGGG-3'. Design and hybridization of variable oligonucleotides (which are gRNA-specific) with a universal reverse oligonucleotide was performed according to (Astre et al.; Harel et al., 2016), and the resulting products were used as a template for in vitro transcription. gRNAs were in vitro transcribed and purified using the MAXIscript T7 kit (ThermoFisher \# AM1312), according to the manufacturer's protocol.

\section{Production of Cas9 mRNA}

Experiments were performed according to (Astre et al., 2022; Harel et al., 2016). The pCS2nCas9n expression vector was used to produce Cas9 mRNA (Addgene, \#47929) (Jao et al., 2013). Capped and polyadenylated Cas 9 mRNA was in vitro transcribed and purified using the mMESSAGE mMACHINE SP6 ULTRA (ThermoFisher \# AM1340).

\section{Single-Stranded DNA Template for Homology-Directed Repair (HDR)}

Homology-directed repair (HDR) experiments for the AK2 gene, a ssDNA template was designed to contain short homology arms ( 20bp) surrounding the gRNA target, and included a novel sequence that replaced the endogenous gRNA target with an NheI restriction site: 5'GTACTGCGTGTGCCACCTGGATTTAGTTGAACGCTAGCGGGCCATGGTGGCATCC GGCTC-3'. The ssDNA template was commercially synthesized and purified prior to injection (QIAquick Nucleotide Removal Kit, QIAGEN) according to (Astre et al., 2022; Harel et al., 2016).

Microinjection of turquoise killifish embryos and generation of mutant fish using CRISPR/Cas9

Microinjection of turquoise killifish embryos was performed according to (Astre et al., 2022; Harel et al., 2016). Briefly, nCas9n-encoding mRNA (300ng/ $\mu \mathrm{L})$ and gRNA (30ng/ $\mu \mathrm{L})$ were 
mixed with phenol-red (P0290, Sigma-Aldrich) and co-injected into one-cell stage fish embryos. Sanger DNA sequencing was used for detecting successful germline transmission on F1 embryos. The genomic area encompassing the targeted site ( 600bp) was PCR-amplified using the following primer sequences: APRT_F: 5'-TTCCCTCTTTACTGACGTCTCA-3'; APRT_R: 5'-GAAAAATTCCCACAGTAAGAATGAA-3' and AK2_F: 5'CCCAGGTTCTCTGTTGCATT-3'; AK2_R: 5'-GCGGTTTCCACACAAGAACT-3'. Fish with desired alleles were maintained as stable lines and further outcrossed to minimize potential off-target effects.

\section{METHOD DETAILS}

\section{Organ isolation}

Individual killifish, according to the specified age, gender, genotype, and feeding condition, were euthanized in $400 \mathrm{mg} / \mathrm{L}$ of Tricaine in system water. Animals were dissected on ice under a binocular stereo microscope (Leica S9E) according to (Astre et al., 2022). Whole livers were harvested, cut in half, and placed in two separate tubes. For each fish, one tube was processed for metabolomic and lipidomic profiling, while the other was processed for RNA sequencing (see below). Following weight measurements, tubes were snap-frozen in liquid nitrogen, and stored in $-80^{\circ} \mathrm{C}$ until all samples were collected. All samples were collected during the morning time, between $9 \mathrm{am}$ to12pm, to reduce the potential confounding effects driven by circadian rhythms.

\section{Lipid and polar metabolic profiling}

\section{Sample Preparation}

Extraction and analysis of lipids and polar metabolites was performed at the Life Sciences Core Facilities, Metabolic Profiling Unit (Weizmann Institute of Science), as previously described in (Malitsky et al., 2016; Salem et al., 2017) with some modifications. Briefly, liver samples were lyophilized, ground to powder and mixed with $1 \mathrm{~mL}$ of a pre-cooled $\left(-20^{\circ} \mathrm{C}\right)$ homogenous methanol:methyl-tert-butyl-ether (MTBE) 1:3 (v/v) mixture, containing the following internal standards: $0.1 \mu \mathrm{g} / \mathrm{mL}$ of Phosphatidylcholine (17:0/17:0) (Avanti), $0.4 \mu \mathrm{g} / \mathrm{mL}$ of Phosphatidylethanolamine (17:0/17:0, $0.15 \mathrm{nmol} / \mathrm{mL}$ of Ceramide/Sphingoid Internal Standard Mixture I (Avanti, LM6005), $0.0267 \mu \mathrm{g} / \mathrm{mL}$ d5-TG Internal Standard Mixture I (Avanti, LM6000) and $0.1 \mu \mathrm{g} / \mathrm{mL}$ Palmitic acid-13C (Sigma, 605573). The tubes were vortexed and then sonicated for $30 \mathrm{~min}$ in ice-cold Transsonic 460/H sonication bath (Elma) at $35 \mathrm{kHz}$ (taken for a brief vortex every $10 \mathrm{~min})$. Then, UPLC-grade water: methanol $(3: 1, \mathrm{v} / \mathrm{v})$ solution $(0.5$ 
$\mathrm{mL}$ ), containing internal polar metabolite standards (C13 and N15 labeled amino acids standard mix, Sigma, 767964) were added to the tubes. Following 5 min centrifugation at maximum speed, the upper organic phase was transferred into $2 \mathrm{~mL}$ Eppendorf tube. The polar phase was re-extracted as described above, with $0.5 \mathrm{~mL}$ of MTBE. Both parts of organic phase were combined and dried (at $21{ }^{\circ} \mathrm{C}$ setting) using a Refrigerated CentriVap Concentrator (Labconco) and then stored at $-80^{\circ} \mathrm{C}$ until analysis. Lower, polar phase, was similarly lyophilized and stored at $-80^{\circ} \mathrm{C}$ until analysis.

\section{LC-MS for lipidomic analysis}

For analysis, the dried lipid extracts were re-suspended in $150 \mu \mathrm{L}$ mobile phase B (see below) and centrifuged again at maximum speed at $4^{\circ} \mathrm{C}$ for $5 \mathrm{~min}$. Lipid extracts were analyzed using a Waters ACQUITY I class UPLC system coupled to a mass spectrometer (Thermo Exactive Plus Orbitrap) which was operated in switching positive and negative ionization mode. The analysis was performed using Acquity UPLC System combined with chromatographic conditions as described in (Malitsky et al., 2016) with small alterations. Briefly, the chromatographic separation was performed on an ACQUITY UPLC BEH C8 column $(2.1 \times 100$ mm, i.d., $1.7 \mu \mathrm{m}$ ) (Waters Corp., MA, USA). The mobile phase A consisted of $45 \%$ water (UPLC grade) with $1 \% 1 \mathrm{M} \mathrm{NH} 4 \mathrm{Ac}, 0.1 \%$ acetic acid, and of $55 \%$ mobile phase B (acetonitrile: isopropanol (7:3) with $1 \% 1 \mathrm{M} \mathrm{NH} 4 \mathrm{Ac}, 0.1 \%$ acetic acid). The column was maintained at $40^{\circ} \mathrm{C}$ and flow rate of mobile phase was $0.4 \mathrm{~mL} / \mathrm{min}$. Mobile phase A was run for $1 \mathrm{~min}$ at $100 \%$, then it was gradually reduced to $25 \%$ at $12 \mathrm{~min}$, following a gradual decrease to $0 \%$ at $16 \mathrm{~min}$. Then, mobile phase B was run at $100 \%$ until 21 min, and mobile phase A was set to $100 \%$ at 21.5 min. Finally, the column was equilibrated at 100\% A until 25 min.

\section{Lipid identification and quantification}

Orbitrap data was analyzed using LipidSearch ${ }^{\mathrm{TM}}$ software (Thermo Fisher Scientific). The validation of the putative identification of lipids was performed by comparing to home-made library which contains lipids produced by various organisms and on the correlation between retention time (RT), carbon chain length, and degree of unsaturation. Relative levels of lipids were normalized to the internal standards and the amount of tissue used for analysis.

\section{LC-MS for polar metabolite analysis}

For metabolic profiling of the polar phase samples, the lyophilized pellets were dissolved using $150 \mu \mathrm{L}$ DDW-methanol (1:1), centrifuged twice (at maximum speed) to remove possible precipitants, and were injected into the LC-MS system. Metabolic profiling of the polar phase 
was performed as described in (Zheng et al., 2015) with minor modifications described below. Briefly, analysis was performed using Acquity I class UPLC System combined with mass spectrometer (Thermo Exactive Plus Orbitrap) which was operated in a negative ionization mode. The LC separation was performed using the SeQuant Zic-pHilic $(150 \mathrm{~mm} \times 2.1 \mathrm{~mm})$ with the SeQuant guard column $(20 \mathrm{~mm} \times 2.1 \mathrm{~mm})$ (Merck). The Mobile phase B was acetonitrile; Mobile phase A consisted of $20 \mathrm{mM}$ ammonium carbonate with $0.1 \%$ ammonia hydroxide in water:acetonitrile (80:20, v/v). The flow rate was kept at $200 \mu \mathrm{L} / \mathrm{min}$ and gradient as follows: $0-2 \min 75 \%$ of $\mathrm{B}, 17 \min 12.5 \%$ of $\mathrm{B}, 17.1 \mathrm{~min} 25 \%$ of $\mathrm{B}, 19 \min 25 \%$ of $\mathrm{B}$, $19.1 \min 75 \%$ of $\mathrm{B}, 23 \min 75 \%$ of $\mathrm{B}$.

\section{Polar metabolites identification and quantification}

The data processing was performed using TraceFinder ${ }^{\mathrm{TM}} 4$ software (Thermo Fisher) and compounds were identified by accurate mass, retention time, isotope pattern, fragments, and verified using in-house mass spectra library.

\section{Mass spectrometry analysis}

Samples were normalized by internal standard and sample weight. Metabolites and lipids were omitted if they were detected in less than $70 \%$ of the samples (19/27 samples), with a final list of 140 metabolites and 787 lipids. Values were log-2 transformed and normalized by the average of each metabolite or lipid. Hierarchical clustering was based on Pearson correlation.

Metabolomics: to perform a statistical analysis we clustered our samples into 4 groups that reflect our experimental design, and performed one-way ANOVA, Tukey post-hoc, and FDR correction. Specifically, experimental groups were clustered according to following (see Figure 3c, left): fully fed (i); young fasted (ii); old WT fasted (iii); and old $A P R T^{48 /+}$ fasted (iv). We were able to cluster all fully-fed conditions together, as a one-way ANOVA test demonstrated their expression levels were highly similar (i.e. only 4/137 metabolites with a significant difference). Using these clusters, we can estimate a normal response to fasting (comparing clusters $\mathrm{i}$ and ii, Tukey post-hoc), and age-dependent deregulated nutrient sensing that is rescued in old $A P R T^{\Delta 8 /+}$ fish (comparing clusters iii and iv, Tukey post-hoc). Significance was called at FDR $\leq 5 \%$ for both ANOVA and Tukey post-hoc. These significant metabolites, and the metabolites the hierarchically clustered with them (displaying similar expression patterns) were selected (Figure S3a, blue highlight), and then further clustered according the metabolic pathways BioCyc (Caspi et al., 2020). 
Lipidomic: Significant lipids could not be detected, as the lipidomic data was more variable across samples. Additionally, we were not able to treat all fully-fed conditions as a single cluster, as 325/787 lipids (41.2\%) displayed significant differences between fully-fed conditions (using ANOVA with FDR correction). Therefore, our statistical test was focused on fasting conditions. We applied one-way ANOVA between 3 clusters: young (i); old WT (ii); and old $A P R T^{\Delta 8 /+}$ (iii). Using these clusters, we estimated age-dependent deregulated nutrient sensing (comparing clusters $\mathrm{i}$ and ii) and rejuvenation in old $A P R T^{\Delta 8 /+}$ fish (comparing clusters ii and iii). Significance was called at FDR $<5 \%$ for ANOVA, and top 5\% for Tukey post-hoc (between groups ii and iii).

\section{LC-MS for direct quantification of AMP, ADP, and ATP}

Metabolites were extracted using standard Methanol/acetonitrile/water, and extracts were snapfrozen. For LC-MS, the Thermo Vanquish Flex ultra-high-performance liquid chromatography (UPLC) system coupled to Orbitrap Exploris 240 Mass Spectrometer (Thermo Fisher Scientific) was used. Resolution was set to 120,000 at 200 mass/charge ratio (m/z) with electrospray ionization and polarity switching mode to enable both positive and negative ions across a mass range of $67-1000 \mathrm{~m} / \mathrm{z}$. The chromatography was performed as described previously (Mackay et al., 2015). Briefly, UPLC setup consisted ZIC-pHILIC column (SeQuant; $150 \mathrm{~mm} \times 2.1 \mathrm{~mm}, 5 \mu \mathrm{m}$; Merck). $5 \mu \mathrm{l}$ of cell extracts were injected and the compounds were separated using a mobile phase gradient of $15 \mathrm{~min}$, starting at $20 \%$ aqueous (20 mM ammonium carbonate adjusted to $\mathrm{pH} 9.2$ with $0.1 \%$ of $25 \%$ ammonium hydroxide): $80 \%$ organic (acetonitrile), and terminated with $20 \%$ acetonitrile. Flow rate and column temperature were maintained at $0.2 \mathrm{ml} / \mathrm{min}$ and $45^{\circ} \mathrm{C}$, respectively, for a total run time of $27 \mathrm{~min}$. All metabolites were detected using mass accuracy below $1 \mathrm{ppm}$. Thermo Xcalibur 4.4 was used for data acquisition. TraceFinder 4.1 was used for data analysis. Peak areas of metabolites were determined by using the exact mass of the single charged ions. The peak areas of different metabolites were determined using Thermo TraceFinderTM 4.1 software, where metabolites were identified by the exact mass of the single charged ion and by known retention time, using an in-house MS library built by running commercial standards of all detected metabolites. For data normalization, raw data files were processed with Compound Discoverer 3.1 to obtain total measurable ions peak intensities for each sample. Each identified metabolite intensity was normalized to the total intensity of the sample. Metabolite-Auto Plotter (Pietzke and Vazquez, 2020) was used for data visualization during data processing.

\section{RNA sequencing}




\section{RNA-seq library preparation}

Organs were isolated as described above. Samples were disrupted by bead beating in $300 \mu 1$ of TriZol (Sigma) and a single 3mm metal bead (Eldan, BL6693003000) using TissueLyzer LT (QIAGEN, \#85600) with a dedicated adaptor (QIAGEN, \#69980). Beating was performed twice at $50 \mathrm{~Hz}$ for 2 minutes. RNA extraction was performed with Direct-zol RNA Purification Kits (Zymo). RNA concentration and quality were determined by using an Agilent 2100 bioanalyser (Agilent Technologies). Library preparation was performed using KAPA mRNA HyperPrep Kit (ROCHE-08105936001) according to the recommended protocols. Library concentrations were measured by Qubit (dsDNA HS, Q32854), and quality was measured by Tape Station (HS, 5067-5584). Libraries were sequenced by NextSeq 500 high output kit V2, 75 cycle single-end (Illumina, 20024906) using a NextSeq 500 machine (Illumina) with 30 million reads per sample.

\section{RNA sequencing analysis}

Quality control and adapter trimming of the fastq sequence files were performed with FastQC (v0.11.8) (Andrews, 2017), fastx-toolkits (v0.0.13) and the Trim Galore! (v0.6.4) (Krueger, 2015) for Cutadapt (3.4)(Martin, 2011). Options were set to remove Illumina TruSeq adapters and end sequences to retain high-quality bases with phred score $>20$ and a remaining length $>20$-bp. Successful processing was verified by re-running FastQC. Reads was mapped and quantified to the killifish genome Nfu_20140520 (Reichwald et al., 2015; Valenzano et al., 2015) using STAR 2.7.6a (Dobin et al., 2013). Differential gene expression as a function of age, genotype, and the interaction between age and genotype was performed using the edgeR package (edgeR 3.32.1) (McCarthy et al., 2012; Robinson et al., 2010). As the three main experimental conditions (males fully fed, males fasted, and female fasted) were sequenced separately, analysis was independently performed on each dataset. Lowly expressed genes were filtered using default parameters in edgeR, and samples were normalized by TMM (Robinson and Oshlack, 2010).

\section{Gene Ontology Enrichment Analysis}

Enriched Gene Ontology (GO) terms associated with transcripts levels (from either old versus young, heterozygous versus wild type, or Genotype-Age interaction analysis) were identified using Gene Set Enrichment Analysis (GSEA) implemented in R package clusterProfiler (version 3.18.1) (Yu et al., 2012). All the transcripts were ranked and sorted in descending order based on multiplication of $\log 2$ transformed fold change and $-\log 10$ (p-value). Note that 
due to random seeding effect in GSEA, the exact p-value and rank of the enriched terms may differ for each run. These random seeds did not qualitatively affect the enrichment analyses. Heatmap visualization of heatmaps on chosen GO term associated to GO human annotation from org.Hs.eg.db (v3.13.0) (Carlson et al., 2019) and AnnotationDbi (v1.54.1) (Pagès et al., 2021) was perform using ComplexHeatmap (v2.8.0) (Gu et al., 2016) with hierarchical clustering using Pearson correlation. The heatmaps were visualized either by standardized $\log 2$-transformed normalized count per million (CPM) with all the associated genes in the GO term (the samples itself or average of each condition) or $\log 2$ fold-change between heterozygote versus wild-type or old versus young on GO term genes with fold-change $>1.5$ in at least one of the conditions. Venn diagram was plotted by VennDiagram (v1.6.20) R package.

\section{Principal component analysis (PCA)}

Standardized $\log 2$-transformed normalized count per million (CPM) were used as input for principal component analysis (PCA). PCA was performed using autoplot function implemented in R package ggfortify (version 0.4.12) and plotted using ggplot2 (version 3.3.5). Survival, maturity, and growth assays

\section{Lifespan measurements}

For reproducible lifespan experiments, constant housing parameters are very important (Astre et al., 2022; Dodzian et al., 2018). Following hatching, fish were raised with a following density control: 10 fish in a 1-liter tank for week 1, 5 fish in a 3-liter tank for weeks 2-4. From this point onwards, adult fish were genotyped and single housed in a 1-liter tanks for their remaining lifespan. Plastic plants were added for enrichment. Both male and female fish were used for lifespan experiments, and were treated identically. Fish mortality was documented daily starting week 4. Lifespan analyses were performed using GraphPad Prism (https://www.graphpad.com/) for all survival curves with a Kaplan-Meier estimator. To compare the survival curves between different experimental groups, we performed log rank test to examine if the survival curves are significantly different.

\section{Maturity and growth measurements}

As fish had to be independently evaluated prior to genotyping, housing was slightly different from lifespan experiments, and fish were individually housed in a 1-liter tank starting week 2. For sexual maturity assay (in males only) coloration status of the fish was visually scored according to the onset of tail coloration. For measuring growth, fish were imaged at the 
indicated timepoints with a Canon Digital camera EOS 250D, prime lens Canon EF 40mm f/2.8 STM that documented body length. To limit vertical movement during imaging, fish were positioned in a water tank with $3 \mathrm{~cm}$ water depth, and images were taken from the top using fixed lighting and height. A ruler was included in each image for accurate scale. Body length was then calculated (using Matlab R2021a), by converting pixel number to centimeters using the included reference ruler.

\section{Fertility Analysis}

Fish fertility was evaluated according to (Harel et al., 2015). Briefly, 5 independent pairs of fish of the indicated genotypes were placed in the same tank, each consisting of one male and one age-matched female. All breeding pairs were allowed to continuously breed on sand trays, and embryos were collected and counted on a weekly basis for 4 weeks. Unfertilized eggs are easily identified, as they die shortly after egg-laying and the yolk becomes opaque. Results were expressed as a ratio of fertilized eggs per week of egg-lay. The 4-week average of eggs collected for each mating pair was considered as one data point. Significance was calculated using unpaired parametric $\mathrm{t}$ test in Prism (GraphPad).

\section{Histology}

Tissues samples were processed according to (Harel et al., 2009, 2012). For paraffine sections, the body cavity of the fish was opened, and following a $72 \mathrm{~h}$ fixation in $4 \%$ PFA solution at $4^{\circ} \mathrm{C}$, samples were paraffin-embedded. Sections of 4-6 $\mu \mathrm{m}$ were stained with hematoxylin and eosin and examined by microscopy. For cryosections, isolated livers were incubated overnight in $4 \%$ PFA at $4{ }^{\circ} \mathrm{C}$, and then placed in $30 \%$ sucrose in DPBS at $4{ }^{\circ} \mathrm{C}$ for cryoprotection until the livers sank (approximately 12h). Livers were then embedded in OCT freezing media and placed in $-20^{\circ} \mathrm{C}$ for $12 \mathrm{~h}$, and section of $14 \mu \mathrm{m}$ were used for downstream applications.

\section{Staining of neutral lipid droplets}

Cryosections were stained for lipids droplets according to (Griffett et al., 2013). Briefly, slides were incubated in $2 \mu \mathrm{g} / \mathrm{mL}$ BODIPY $493 / 503$ (Invitrogen $^{\mathrm{TM}}$ ) in 1X DPBS for $15 \mathrm{~min}$. Sections were washed three times in cold $1 \mathrm{X}$ DPBS and then counterstained with $1 \mu \mathrm{g} / \mathrm{mL}$ Hoechst. Three sections per liver, from a total of 3-4 fish, were imaged using the FV-1200 confocal microscope (Olympus, Japan). BODIPY intensity was normalized by nuclear staining (Parafati et al., 2018), quantified by ImageJ, and values were analyzed with GraphPad Prism.

\section{Dietary manipulations}




\section{$24 h$ fasting}

Following the morning feeding, fish were fasted for 24 hours.

\section{Lifelong intermitted fasting}

Starting on week 4, fish were fed once a day for their remaining lifespan with a combination of GEMMA Micro 300 Fish Diet (Skretting Zebrafish, USA) supplemented by live Artemia.

\section{Short-term high-fat diet}

Old fish (15 weeks old) were fed twice a day, for a total of one week, with BioMar fish diet (0.5mm INICIO Plus SEA BREAM, BioMar Group, Demark), supplemented by live Artemia. This protocol was adapted from a recent report that demonstrated that killifish fed with BioMar INICIO had more visceral fat, and their livers possessed more lipid droplets (Žák et al., 2020). As demonstrated for other short-term approaches in zebrafish (Landgraf et al., 2017), one week of feeding with BioMar was sufficient for inducing significant accumulation of lipid droplets in the liver (when compared with our normal feeding, Figure S7f).

\section{Antibodies validation}

To validate antibody specificity using western blot, we generated two conditions in which AMPK-related pathways are expected to be altered. Specifically, male killifish were either starved for 3 days (Mohapatra et al., 2020) or exposed to 20mM 2 deoxy glucose for $2 \mathrm{~h} 30$ in system water (2-DG, Sigma Aldrich) (LANE et al., 1998).

\section{7ß-estradiol (E2) exposure}

$17 \beta$-estradiol (E2, Holland Moran) was dissolved in ethanol, and sprayed on food pellets at $100 \mathrm{mg} / \mathrm{kg}$ for both drugs (Mohapatra et al., 2020). Food was then dried overnight under a chemical fume hood. Adult fish ( 9 weeks old) were individualized in tanks containing system water, and fed twice a day for a period of 8 days. Control groups were fed with ethanol treated food pellets and were similarly maintained and sampled. As expected, in response to the 17ß-estradiol treatment, we observed lipid accumulation in treated fish (Cakmak et al., 2006).

\section{Generation of primary fibroblast cultures from killifish tail fins}

Adult fish (8-12 weeks old), of the indicated genotype and gender were sedated with MS-222 (200mg/L Tricaine, in system water). All following experiments were conducted at $28^{\circ} \mathrm{C}$ unless stated otherwise. Following sedation, a $2-3 \mathrm{~mm}$ tissue was trimmed from the tail fin using a 
sterile razor blade, and individually disinfected for 10min with a 25ppm iodine solution (PVPI, Holland Moran 229471000) in DPBS (Biological Industries). Followed by a rinse with DPBS, tissue samples were incubated for $2 \mathrm{~h}$ with $1 \mathrm{~mL}$ of an antibiotic solution containing Gentamicin $\left(50 \mu \mathrm{g} / \mathrm{mL}\right.$ Gibco) and Primocin ${ }^{\mathrm{TM}}(50 \mu \mathrm{g} / \mathrm{mL}$, InvivoGen) in DPBS at room temperature. Tissues were then transferred into an enzymatic digestion buffer $(200 \mu \mathrm{L}$, in a $24-$ well plate) containing Collagenase Type P $(0.4 \mathrm{mg} / \mathrm{mL}$, Merck Millipore) in Leibovitz's L-15 Medium (Gibco), and mechanical dissociation was applied with a sterile pair of scissors. Crudely dissociated tissue was placed in a 6-well plate. Liquid was gently aspirated, and tissue was incubated for one hour, to better attach. Next, $400 \mu \mathrm{L}$ of complete Leibovitz's L-15 growth medium (Gibco) was gently added, supplemented with Fetal Bovine Serum (15\% FBS, Gibco), penicillin/streptomycin (50 U/ml, Gibco), Gentamicin (Gibco, 50 $\mu \mathrm{g} / \mathrm{ml}$ ) and Primocin ${ }^{\mathrm{TM}}$ $(50 \mu \mathrm{g} / \mathrm{ml}$, InvivoGen), and incubated for several days, until cell migration from the explant was visible. At this stage, they were passaged with Trypsin-EDTA $0.05 \%$ (0.25\% TrypsinEDTA, diluted in DPBS). Cells were incubated at $28^{\circ} \mathrm{C}$ humidified incubator (Binder, Thermo Scientific) with normal air composition, and were used for downstream applications between passages 4-12.

\section{Seahorse Metabolic Extracellular Flux Profiling}

The Seahorse XFp Analyzer (Agilent Technologies, USA) was used to measure oxygen consumption rates (OCR, in $\mathrm{pmol}_{2}$ per minute), and extracellular acidification rates (ECAR, in millipH per minute). Primary fibroblasts from killifish tail fins were cultured as described above. The day before the experiment, cells were seeded (50,000 cells/well) in XFp 8-well plates (Agilent Technologies, USA). A relatively large number of cells was used in our assay to compensate for the small size and low basal respiration of primary fish cells. All the required drugs were supplied by the manufacturer (Seahorse Bioscience), and drug injection times were according to the XFp standard protocol. OCR and ECAR measurements were analyzed according to manufacturer's instructions and GraphPad Prism (v7, GraphPad).

Although cells were seeded in equal numbers to facilitate OCR and ECAR normalization, actual number of cells in each well was further confirmed using imaging to account for potential differences in proliferation rates. Briefly, following each metabolic assay, cells were fixed and stained for $15 \mathrm{~min}$ with $4 \%$ paraformaldehyde and $10 \mu \mathrm{M}$ Hoechst 33342 (Thermo Fisher) in DPBS. Each well was imaged three times (IX83, Olympus), and averaged cell density (number of cells per field of view) was counted using CellProfiler (https://cellprofiler.org). Finally, the total number of cells per well was estimated by the relative 
area represented by the field of view, compared to the total area of the well. The following assays were performed 2-3 times. Each experiment contains at least three independent samples per genotype.

\section{Cell Mito Stress Assay}

Cell Mito Stress Test (XFp Cell Mito Stress Test Kit, Agilent Technologies) was performed following the manufacturers guidelines, with minor adaptations for fish cells described below. 1 hour before the measurements, culture medium was replaced and the cells were incubated for $60 \mathrm{~min}$ at $28^{\circ} \mathrm{C}$ with the Seahorse XF Base medium (Seahorse Bioscience), supplemented with $2 \mathrm{mM}$ L-glutamine and $1 \mathrm{mM}$ pyruvate (Seahorse Bioscience). Instead of glucose, we used $5 \mathrm{mM}$ galactose (Sigma-Aldrich), which is the sugar source used by the L15 culture media. Oxygen consumption rate (OCR) and Extracellular Acidification Rate (ECAR) were detected after injection of oligomycin (1 $\mu \mathrm{M})$, Carbonyl cyanide-p-trifluoromethoxyphenylhydrazone (FCCP, $1 \mu \mathrm{M})$, and the combination of rotenone \& antimycin A (Rot/AA, $1 \mu \mathrm{M})$.

\section{Cell Glycolysis Stress Assay}

Cell Glycolysis Stress Test (XFp Glycolysis Stress Test Kit, Agilent Technologies) was performed following the standard protocol. Culture media was replaced by Seahorse XF Base medium, supplemented with $2 \mathrm{mM}$ L-glutamine. During the test, final concentration of $15 \mathrm{mM}$ glucose, $1 \mu \mathrm{M}$ oligomycin, and $50 \mathrm{mM}$ of 2-deoxyglucose (Seahorse Bioscience) were injected according to the XFp standard protocol.

\section{Pharmacological manipulation of cultured cells}

\section{Adenine treatment}

Cells were seeded in XFp 8-well plates (Agilent Technologies, USA) at a density of $50,000 \mathrm{cell} /$ well and cultured overnight with adenine $10 \mu \mathrm{M}$. One hour prior to the seahorse experiment, culture medium was replaced with the Seahorse XF Base medium (Agilent Technologies, USA) supplemented with adenine 10 $\mu \mathrm{M}$ (Sigma Aldrich), and cells were incubated for $60 \mathrm{~min}$ at $28^{\circ} \mathrm{C}$.

\section{8-azaadenine treatment}

To characterize and validate the relative loss of APRT activity in heterozygous cells, an adenine analog, 8-azaadenine (Holland Moran) was used according to (Jones and Sargent, 1974). Briefly, mutant APRT cells that have reduced ability to metabolize adenine exhibit partial resistance to the toxic intermediate generated by AA (Jones and Sargent, 1974). Cells were 
treated with a range of concentrations of AA $(0-200 \mu \mathrm{g} / \mathrm{mL})$ for 4 days without media change. As $\mathrm{AA}$ is dissolved in $1 \mathrm{M} \mathrm{NaOH}$, an equal amount of $1 \mathrm{M} \mathrm{NaOH}$ was added to the controls. Experiment were performed twice using 3 independent replicates per genotype.

\section{Serum starvation}

Cells were seeded in a 12-well plate at a density of 100,000 cells/well. The following day, the medium was replaced with fresh media for $4 \mathrm{~h}$, with L-15 medium containing either or $15 \%$ (control) or 5\%FBS.

\section{Mitochondrial morphology}

\section{Fluorescence microscopy}

Cells were plated on a $\mu$-Slide 8 Well Glass Bottom plates (ibidi, \#80827), at a density of 40000cells/well. Confocal imaging was performed on live cells using a sealed, environmentally controlled chamber without $\mathrm{CO}_{2}$ at $28^{\circ} \mathrm{C}$. A day after, cells were labeled with 200nM Mitoview 633 (Biotium) for 15min prior to live imaging. Labeled cells were imaged on a laser scanning confocal microscope (FV-1200, Olympus, Japan) using a 60X/1.42 oil immersion objective. Scans were acquired using a sequential mode. All fluorophores were excited on separate tracks. Hoechst was excited with $405 \mathrm{~nm}$ and emission was captured through $485 \mathrm{~nm}$ short-pass filter. Mitoview 633 was excited with $561 \mathrm{~nm}$ and emission was captured through a 570-620 nm.

\section{Mitochondrial footprint and network analysis}

To analyze mitochondrial morphology we used MiNA, a plug-in macro toolset for Fiji (Schindelin et al., 2012). This workflow estimates mitochondrial footprint from a binarized copy of the provided image, and the lengths of mitochondrial networks are estimated using a topological skeleton (mitochondrial length was defined as the length of rods and network branches). Additionally, we used the generated overlays (or a 3D rendering) to further confirm the accuracy of the analysis. Experiment were performed twice using 3 independent replicates per genotype. Three field of views, containing between $4-8$ cells, were independently analyzed as described above.

\section{Protein Gel Electrophoresis and Immunoblotting}

\section{Liver samples}

Individual killifish, according to the specified age, gender, genotype, and feeding condition, were euthanized in $400 \mathrm{mg} / \mathrm{L}$ of Tricaine in system water. Animals were dissected on ice under a stereo microscope (Leica S9E) according to (Astre et al., 2022). All procedures were carried 
at $4^{\circ} \mathrm{C}$ unless stated otherwise. Homogenization was performed in a $2 \mathrm{~mL}$ Safe-Lock Tubes (Eppendorf), to allow full homogenization of liver tissue, using 3mm-metal beads (Eldan Israel, Cat\# RC55 420) and RIPA lysis buffer (7.9 g/L Tris-HCl, 9g/L NaCl, 0.76 g/L EGTA, 10 ml $10 \%$ Triton $\mathrm{X}-100, \mathrm{pH} 7.2$ ). Prior to homogenization, $200 \mu \mathrm{l}$ buffer was added to $\sim$ half a liver, and freshly supplemented with anti-protease and anti-phosphatase cocktail (Biotool). Homogenization was carried out with mechanical disruption using TissueLyzer LT (QIAGEN, \#85600) with a dedicated adaptor (QIAGEN, \#69980) at 50Hz for 2 minutes X2. Protein concentration was measured with Pierce ${ }^{\mathrm{TM}}$ BCA Protein Assay Kit (Thermo Scientific ${ }^{\mathrm{TM}}$ ), according to manufacture instructions. $5-10 \mu \mathrm{g}$ of liver homogenate was then mixed with the Sample Buffer (Tris-HCl pH6.8 62.5mM, Glycerol 10\%, SDS 2\%) at a ratio of 1:3. 2mercaptoethanol (Sigma Aldrich) (5\%) and Bromophenol blue (Sigma Aldrich) (0.02\%) were freshly added, boiled at $95^{\circ} \mathrm{C}$ for 10 minutes, and placed on ice.

\section{Primary culture}

Following a wash with cold DPBS, cells were re-suspended with $250 \mu 1$ of the Sample Buffer (Tris-HCl pH6.8 62.5mM, Glycerol 10\%, SDS 2\%) at a ratio of 1:3, freshly supplemented with 5\% 2-mercaptoethanol (Sigma Aldrich), 0.02\% Bromophenol blue (Sigma Aldrich), and antiprotease and anti-phosphatase cocktail (Biotool). Samples were then boiled at $95^{\circ} \mathrm{C}$ for 10 minutes. $10 \mu \mathrm{l}$ were used for western blot.

\section{Immunoblotting}

Standard western blot was performed. Briefly, protein extracted $(5-10 \mu \mathrm{g}$ from livers, or $10 \mu \mathrm{l}$ from cells) were resolved using Novex WedgeWell 4-20\% Tris-Glycine gel (XP04205BOX, Thermo Fisher) and electrophoresed in Cell SureLock (Novex) in constant voltage of 50V for 15 min (to clear stacking), followed by $120 \mathrm{~V}$ for $1 \mathrm{~h}$. Transfer to a Nitrocellulose membrane was performed using the iBlot 2 (Thermo Scientific, IB21001), followed by Ponceau Red red staining (Sigma Aldrich, P7170-1L). Membranes were washed in TBST 0.1\% (Tris-HCl 10mM $\mathrm{pH} 8.0, \mathrm{NaCl} 150 \mathrm{mM}$, Tween $0.1 \%$ ) once for $5 \mathrm{~min}$ to remove Ponceau staining, and blocked in 5\% BSA (Sigma-Aldrich) in TBST for 1 hour at room temperature. The resolved proteins were probed with the following antibodies at a concentration of 1:1000 (diluted in blocking solution at $4^{\circ} \mathrm{C}$ overnight): anti-AMPK $\alpha$ (CST-5831), anti-Phospho-AMPK $\alpha$ (Thr172) (CST2535), anti-ACC (CST-3676), anti-Phospho-ACC (CST-3661), anti-S6 Ribosomal Protein (CST-2217), anti-Phospho-S6 Ribosomal Protein (CST-2211), and anti-Actin (MP Bio 0869100). Following three washes of 10min, membranes were incubated with 1:5000 HRP- 
conjugated goat anti-rabbit (ab6721, Abcam) or goat anti-mouse (ab6789, Abcam) antibodies for 1hour, andwashed three times for 10min. Chemiluminescence was detected using EZ-ECL kit (Biological Industry) and imaged with either ChemiDoc MP Imaging System (Biorad) or Fusion Pulse 6 (Vilbert Loumat). Band densitometry was quantified using ImageJ, and normalized according to Actin values.

DNA isolation and measurement of mitochondrial DNA content using quantitative PCR Quantification was performed according to (Hartmann et al., 2011). Briefly, total DNA from cells, tails and liver was extracted using QIAamp Micro kit (Qiagen) according to the manufacturers protocol. The relative mitochondrial DNA (mtDNA) copy number was determined by quantitative PCR (qPCR) with the nuclear $\mathrm{Cdkn} 2 \mathrm{a} / \mathrm{b}$ gene and the mitochondrial 16S rRNA gene (primer sequences are available at (Hartmann et al., 2011)). qPCR was performed with Magnetic Induction Cycler (Mic, bio molecular systems) using Fast SYBR Green Master Mix (2X) (ThermoFisher 4385610), 100nM of each primer, and 20ng DNA as template. All reactions included negative controls (without template, or without the enzyme). $\mathrm{Ct}$ values of mtDNA were normalized to $\mathrm{Ct}$ values of the nuclear locus according to (Hartmann et al., 2011), using the following equation: relative mtDNA copy number per diploid cell= $2 \times 2^{\Delta \mathrm{Ct}}$, where $\Delta \mathrm{Ct}$ is $\mathrm{Ct}_{\mathrm{Cdkn} 2 \mathrm{a} / \mathrm{b} \text { locus }}-\mathrm{Ct}_{\text {mitochondrial locus. }}$

\section{A-to-I RNA editing}

A-to-I editing is the most prevalent RNA editing. It is performed by double-stranded RNAspecific adenosine deaminase (ADAR) family of proteins (Eisenberg and Levanon, 2018). Sequencing machines recognize Inosine as Guanosine, thus allowing us to quantify A-to-I editing by counting mismatches from Adenosine to Guanosine (A2G) ${ }^{2}$.

\section{Quality control for RNA editing}

Additional filtration steps, in addition to those performed for differential gene expression, were taken to eliminate common biases in RNA Editing. Specifically, we used PRINSEQ-lite 0.20.4 (Schmieder and Edwards, 2011) to remove exact duplicates, retain reads no shorter than $72 \mathrm{bp}$, and trim reads longer than $63 \mathrm{bp}$. Duplicate RNA reads, defined as reads with the same sequence on the same strand or with the reverse-compliment sequence on the opposite strand, can result from PCR cycles conducted before sequencing. Using only reads with similar lengths allowed us to compare the RNA Editing Index of different samples. Trimmomatic 0.39 (Bolger et al., 2014) was used to remove Nextera transposase adapters. Finally, we assessed the quality of the reads with FastQC 0.11.8 (Andrews, 2017) and MultiQC 1.11 (Ewels et al., 2016). 


\section{Genome and genomic annotations}

Reads was mapped and quantified to the killifish genome Nfu_20140520 (Reichwald et al., 2015; Valenzano et al., 2015). As ADAR target editing sites are usually prevalent in repetitive regions (Eisenberg and Levanon, 2018), we created a comprehensive annotation of these regions in the killifish genome by expanding the initial annotation. Specifically, we extracted the RepeatMaskerLib.embl library database using RepeatMasker 4.1.0 (Chen, 2004), and identified coding regions using the available GFF file. Finally, we used EDTA 1.9.6 (Ou et al., 2019) to create an extended library of repeats.

\section{Signal-to-noise ratio (SNR)}

Considering A-to-G editing as Signal and the $2^{\text {nd }}$ most prevalent DNA-RNA mismatch other than A-to-G as Noise, we define SNR as:

$$
\text { SNR }=\frac{\text { Signal }}{\text { Signal }+ \text { Noise }} * 100
$$

\section{Hyper editing \& cluster screening}

ADAR's activity is characterized by dense clusters of editing sites, which results in reads that are difficult to align by regular alignment procedures. Therefore, we applied an adaptation to successfully align those hyper-edited reads to the genome (Porath et al., 2014). The output of this method is a list of hyper-edited sites and ultra-edited regions (UE). To get a clear SNR as possible, we applied a previously published approach (Buchumenski et al., 2021), termed here as Cluster Screening. Using a distance of $20 \mathrm{bp}$, we first required that two different editing sites (e.g., A2G and C2T) cannot reside within 20 bp next to each other. Second, each editing site must have a neighbor editing site of the same type, located no further than $20 \mathrm{bp}$ from him. The first demand is meant to overcome alignment errors that are the result of duplication events. The second demand helps to identify clusters of editing sites, contrary to SNPs that occur randomly at various genomic locations. We dismissed editing sites that fail to satisfy either of these requirements.

\section{RNA editing index}

To quantify the global editing levels of each UE cluster, we ran the RNA Editing Index tool (Roth et al., 2019) . For each kind of mismatch from Reference Base to Mutated Base, we define the Index of a region as

$$
\text { Index }=\frac{\text { Mutated Base }}{\text { Mutated Base }+ \text { Reference Base }} * 100
$$


where Reference Base is the number of reads mapped to genomic positions (of that base), and Mutated Base is the number of reads with Reference Base to Mutated Base mismatch mapped to those positions. Specifically, the Index is a weighted average of RNA editing levels of a mismatch across a region. Using the Index allows us to compare RNA editing of different regions and samples. We ran the RNA Editing Indexer (https://github.com/a2iEditing/RNAEditingIndexer) with the following parameters as input: (1) The average expression level of each gene, as quantified by Salmon v1.4 (Patro et al., 2017); (2) RefSeq annotations of the genes and aligned BAM files. Independently of the Hyper Editing tool alignment, we aligned the FastQ files to the genome using BWA 0.7.17 (Li and Durbin, 2009) with the MEM algorithm; (3) The UE clusters we previously chose with Cluster Screening. We ran the Index both in stranded and unstranded mode, but as the SNR of the stranded runs was lower (data not shown), we decided to use its unstranded output. Independent t-tests were done using scipy.stats.ttest_ind 1.6.2, multiple independent t-tests were corrected using statsmodels.stats.multitest.fdrcorrection 0.12.2 using default parameters. 


\section{References}

Adams, J.D., Lauterburg, B.H., and Mitchell, J.R. (1983). Plasma glutathione and glutathione disulfide in the rat: regulation and response to oxidative stress. J. Pharmacol. Exp. Ther. 227, 749-754.

Ahuja, G., Bartsch, D., Yao, W., Geissen, S., Frank, S., Aguirre, A., Russ, N., Messling, J.E., Dodzian, J., Lagerborg, K.A., et al. (2019). Loss of genomic integrity induced by lysosphingolipid imbalance drives ageing in the heart. EMBO Rep 20.

Andrews, S. (2017). FastQC: a quality control tool for high throughput sequence data. 2010. Apfeld, J., O’Connor, G., McDonagh, T., DiStefano, P.S., and Curtis, R. (2004). The AMPactivated protein kinase AAK-2 links energy levels and insulin-like signals to lifespan in C. elegans. Genes Dev. 18, 3004-3009.

Ashpole, N.M., Logan, S., Yabluchanskiy, A., Mitschelen, M.C., Yan, H., Farley, J.A., Hodges, E.L., Ungvari, Z., Csiszar, A., and Chen, S. (2017). IGF-1 has sexually dimorphic, pleiotropic, and time-dependent effects on healthspan, pathology, and lifespan. Geroscience 39, 129-145.

Astre, G., Moses, E., and Harel, I. (2022). The African turquoise killifish (Nothobranchius furzeri): biology and research applications. In Laboratory Fish in Biomedical Research, (Elsevier), pp. 245-287.

Austad, S.N. (2006). Why women live longer than men: sex differences in longevity. Gend. Med. 3, 79-92.

Austad, S.N. (2011). Sex differences in longevity and aging. Handb. Biol. Aging 479-495.

Austad, S.N., and Fischer, K.E. (2016). Sex differences in lifespan. Cell Metab. 23, 10221033.

Baar, E.L., Carbajal, K.A., Ong, I.M., and Lamming, D.W. (2016). Sex-and tissue-specific changes in mTOR signaling with age in C57 BL/6J mice. Aging Cell 15, 155-166.

Balla, T. (2013). Phosphoinositides: tiny lipids with giant impact on cell regulation. Physiol. Rev. 93, 1019-1137.

Ballard, J.W.O., Melvin, R.G., Miller, J.T., and Katewa, S.D. (2007). Sex differences in survival and mitochondrial bioenergetics during aging in Drosophila. Aging Cell 6, 699-708.

Baumgart, M., Priebe, S., Groth, M., Hartmann, N., Menzel, U., Pandolfini, L., Koch, P., Felder, M., Ristow, M., Englert, C., et al. (2016). Longitudinal RNA-Seq Analysis of Vertebrate Aging Identifies Mitochondrial Complex I as a Small-Molecule-Sensitive Modifier of Lifespan. Cell Syst 2, 122-132.

Benayoun, B.A., Pollina, E.A., Singh, P.P., Mahmoudi, S., Harel, I., Casey, K.M., Dulken, B.W., Kundaje, A., and Brunet, A. (2019). Remodeling of epigenome and transcriptome landscapes with aging in mice reveals widespread induction of inflammatory responses. Genome Res 29, 697-709.

Bennett, C.F., Kwon, J.J., Chen, C., Russell, J., Acosta, K., Burnaevskiy, N., Crane, M.M., Bitto, A., Vander Wende, H., and Simko, M. (2017). Transaldolase inhibition impairs mitochondrial respiration and induces a starvation-like longevity response in Caenorhabditis elegans. PLoS Genet. 13, e1006695.

Besse-Patin, A., Léveillé, M., Oropeza, D., Nguyen, B.N., Prat, A., and Estall, J.L. (2017). Estrogen signals through peroxisome proliferator-activated Receptor- $\gamma$ coactivator $1 \alpha$ to 
reduce oxidative damage associated with diet-induced fatty liver disease. Gastroenterology $152,243-256$.

Boitz, J.M., Ullman, B., Jardim, A., and Carter, N.S. (2012). Purine salvage in Leishmania: complex or simple by design? Trends Parasitol. 28, 345-352.

Bolger, A.M., Lohse, M., and Usadel, B. (2014). Trimmomatic: a flexible trimmer for Illumina sequence data. Bioinformatics 30, 2114-2120.

Bollée, G., Harambat, J., Bensman, A., Knebelmann, B., Daudon, M., and Ceballos-Picot, I. (2012). Adenine phosphoribosyltransferase deficiency. Clin. J. Am. Soc. Nephrol. 7, 15211527.

Borras, C., Gambini, J., and Vina, J. (2007). Mitochondrial oxidant generation is involved in determining why females live longer than males. Front Biosci 12, 1008-1013.

Bridges, D., and Saltiel, A.R. (2015). Phosphoinositides: Key modulators of energy metabolism. Biochim. Biophys. Acta (BBA)-Molecular Cell Biol. Lipids 1851, 857-866.

Buchumenski, I., Holler, K., Appelbaum, L., Eisenberg, E., Junker, J.P., and Levanon, E.Y. (2021). Systematic identification of A-to-I RNA editing in zebrafish development and adult organs. Nucleic Acids Res. 49, 4325-4337.

Burkewitz, K., Zhang, Y., and Mair, W.B. (2014). AMPK at the nexus of energetics and aging. Cell Metab. 20, 10-25.

Cakmak, G., Togan, I., and Severcan, F. (2006). 17ß-Estradiol induced compositional, structural and functional changes in rainbow trout liver, revealed by FT-IR spectroscopy: a comparative study with nonylphenol. Aquat. Toxicol. 77, 53-63.

Camici, M., Allegrini, S., and Tozzi, M.G. (2018). Interplay between adenylate metabolizing enzymes and AMP-activated protein kinase. FEBS J. 285, 3337-3352.

Carling, D. (2017). AMPK signalling in health and disease. Curr. Opin. Cell Biol. 45, 31-37.

Carlson, M., Falcon, S., Pages, H., and Li, N. (2019). org. Hs. eg. db: Genome wide annotation for Human. R Packag. Version 3.

Cellerino, A., Valenzano, D.R., and Reichard, M. (2015). From the bush to the bench: the annual Nothobranchius fishes as a new model system in biology. Biol Rev Camb Philos Soc.

Chen, N. (2004). Using Repeat Masker to identify repetitive elements in genomic sequences. Curr. Protoc. Bioinforma. 5, 4-10.

Curtis, R., O’Connor, G., and DiStefano, P.S. (2006). Aging networks in Caenorhabditis elegans: AMP-activated protein kinase (aak-2) links multiple aging and metabolism pathways. Aging Cell 5, 119-126.

Dhir, A., Dhir, S., Borowski, L.S., Jimenez, L., Teitell, M., Rötig, A., Crow, Y.J., Rice, G.I., Duffy, D., and Tamby, C. (2018). Mitochondrial double-stranded RNA triggers antiviral signalling in humans. Nature 560, 238-242.

Dobin, A., Davis, C.A., Schlesinger, F., Drenkow, J., Zaleski, C., Jha, S., Batut, P., Chaisson, M., and Gingeras, T.R. (2013). STAR: ultrafast universal RNA-seq aligner. Bioinformatics $29,15-21$.

Edvardsson, V.O., Goldfarb, D.S., Lieske, J.C., Beara-Lasic, L., Anglani, F., Milliner, D.S., and Palsson, R. (2013). Hereditary causes of kidney stones and chronic kidney disease.

Pediatr. Nephrol. 28, 1923-1942. 
Eisenberg, E., and Levanon, E.Y. (2018). A-to-I RNA editing-immune protector and transcriptome diversifier. Nat. Rev. Genet. 19, 473-490.

Engle, S.J., Stockelman, M.G., Chen, J., Boivin, G., Yum, M.-N., Davies, P.M., Ying, M.Y., Sahota, A., Simmonds, H.A., and Stambrook, P.J. (1996). Adenine phosphoribosyltransferase-deficient mice develop 2, 8-dihydroxyadenine nephrolithiasis. Proc. Natl. Acad. Sci. 93, 5307-5312.

Evan, A.P., Bledsoe, S.B., Connors, B.A., Deng, L., Liang, L., Shao, C., Fineberg, N.S., Grynpas, M.D., Stambrook, P.J., and Youzhi, S. (2001). Sequential analysis of kidney stone formation in the Aprt knockout mouse. Kidney Int. 60, 910-923.

Ewels, P., Magnusson, M., Lundin, S., and Käller, M. (2016). MultiQC: summarize analysis results for multiple tools and samples in a single report. Bioinformatics 32, 3047-3048.

Finkel, T. (2015). The metabolic regulation of aging. Nat. Med. 21, 1416.

Di Florio, D.N., Sin, J., Coronado, M.J., Atwal, P.S., and Fairweather, D. (2020). Sex differences in inflammation, redox biology, mitochondria and autoimmunity. Redox Biol. 31, 101482.

Folgueras, A.R., Freitas-Rodriguez, S., Velasco, G., and Lopez-Otin, C. (2018). Mouse Models to Disentangle the Hallmarks of Human Aging. Circ Res 123, 905-924.

Fontana, L., Partridge, L., and Longo, V.D. (2010). Extending healthy life span--from yeast to humans. Science (80-. ). 328, 321-326.

Franceschi, C., Garagnani, P., Parini, P., Giuliani, C., and Santoro, A. (2018). Inflammaging: a new immune-metabolic viewpoint for age-related diseases. Nat. Rev. Endocrinol. 14, 576590 .

Garcia, D., and Shaw, R.J. (2017). AMPK: mechanisms of cellular energy sensing and restoration of metabolic balance. Mol. Cell 66, 789-800.

Garcia, D., Hellberg, K., Chaix, A., Wallace, M., Herzig, S., Badur, M.G., Lin, T., Shokhirev, M.N., Pinto, A.F.M., and Ross, D.S. (2019). Genetic liver-specific AMPK activation protects against diet-induced obesity and NAFLD. Cell Rep. 26, 192-208.

Graf, M., Hartmann, N., Reichwald, K., and Englert, C. (2013). Absence of replicative senescence in cultured cells from the short-lived killifish Nothobranchius furzeri. Exp Gerontol 48, 17-28.

Green, C.L., Lamming, D.W., and Fontana, L. (2021). Molecular mechanisms of dietary restriction promoting health and longevity. Nat. Rev. Mol. Cell Biol. 1-18.

Greer, E.L., Dowlatshahi, D., Banko, M.R., Villen, J., Hoang, K., Blanchard, D., Gygi, S.P., and Brunet, A. (2007). An AMPK-FOXO pathway mediates longevity induced by a novel method of dietary restriction in C. elegans. Curr. Biol. 17, 1646-1656.

Gu, Z., Eils, R., and Schlesner, M. (2016). Complex heatmaps reveal patterns and correlations in multidimensional genomic data. Bioinformatics 32, 2847-2849.

Hägg, S., and Jylhävä, J. (2021). Sex differences in biological aging with a focus on human studies. Elife 10, e63425.

Hamilton, D.J., Minze, L.J., Kumar, T., Cao, T.N., Lyon, C.J., Geiger, P.C., Hsueh, W.A., and Gupte, A.A. (2016). Estrogen receptor alpha activation enhances mitochondrial function and systemic metabolism in high-fat-fed ovariectomized mice. Physiol. Rep. 4, e12913.

Hardie, D.G., Ross, F.A., and Hawley, S.A. (2012). AMPK: a nutrient and energy sensor that 
maintains energy homeostasis. Nat. Rev. Mol. Cell Biol. 13, 251-262.

Harel, I., and Brunet, A. (2015). The African Turquoise Killifish: A Model for Exploring Vertebrate Aging and Diseases in the Fast Lane. Cold Spring Harb Symp Quant Biol.

Harel, I., Benayoun, B.A., Machado, B., Singh, P.P., Hu, C.K., Pech, M.F., Valenzano, D.R., Zhang, E., Sharp, S.C., Artandi, S.E., et al. (2015). A platform for rapid exploration of aging and diseases in a naturally short-lived vertebrate. Cell.

Harel, I., Valenzano, D.R., and Brunet, A. (2016). Efficient genome engineering approaches for the short-lived African turquoise killifish. Nat. Protoc.

Horstman, A.M., Dillon, E.L., Urban, R.J., and Sheffield-Moore, M. (2012). The role of androgens and estrogens on healthy aging and longevity. Journals Gerontol. Ser. A Biomed. Sci. Med. Sci. 67, 1140-1152.

Jenniskens, F.A., Jopperi-Davis, K.S., Walters, L.C., Schorr, E.N., Rogers, L.K., Welty, S.E., and Smith, C. V (2002). Effects of fasting on tissue contents of coenzyme A and related intermediates in rats. Pediatr. Res. 52, 437-442.

Johnson, A.A., and Stolzing, A. (2019). The role of lipid metabolism in aging, lifespan regulation, and age-related disease. Aging Cell 18, e13048.

Johnson, T.A., Jinnah, H.A., and Kamatani, N. (2019). Shortage of cellular ATP as a cause of diseases and strategies to enhance ATP. Front. Pharmacol. 10, 98.

Jones, G.E., and Sargent, P.A. (1974). Mutants of cultured Chinese hamster cells deficient in adenine phosphoribosyl transferase. Cell 2, 43-54.

Justo, R., Boada, J., Frontera, M., Oliver, J., Bermúdez, J., and Gianotti, M. (2005). Gender dimorphism in rat liver mitochondrial oxidative metabolism and biogenesis. Am. J. Physiol. Physiol. 289, C372-C378.

Kane, A.E., Sinclair, D.A., Mitchell, J.R., and Mitchell, S.J. (2018). Sex differences in the response to dietary restriction in rodents. Curr. Opin. Physiol. 6, 28-34.

Kim, Y., Nam, H.G., and Valenzano, D.R. (2016). The short-lived African turquoise killifish: an emerging experimental model for ageing. Dis Model Mech 9, 115-129.

Klinge, C.M. (2008). Estrogenic control of mitochondrial function and biogenesis. J. Cell. Biochem. 105, 1342-1351.

Kristensen, T.N., Loeschcke, V., Tan, Q., Pertoldi, C., and Mengel-From, J. (2019). Sex and age specific reduction in stress resistance and mitochondrial DNA copy number in Drosophila melanogaster. Sci. Rep. 9, 1-7.

Krueger, F. (2015). Trim galore. A Wrapper Tool around Cutadapt FastQC to Consistently Apply Qual. Adapt. Trimming to FastQ Files 516, 517.

L Ipata, P., Camici, M., Micheli, V., and G Tozzi, M. (2011). Metabolic network of nucleosides in the brain. Curr. Top. Med. Chem. 11, 909-922.

LANE, M.A., INGRAM, D.K., and ROTH, G.S. (1998). 2-Deoxy-D-glucose feeding in rats mimics physiologic effects of calorie restriction. J. Anti. Aging. Med. 1, 327-337.

Lee, M.H., Malloy, C.R., Corbin, I.R., Li, J., and Jin, E.S. (2019). Assessing the pentose phosphate pathway using [2, 3-13C2] glucose. NMR Biomed. 32, e4096.

Lee, S.S., Lee, R.Y.N., Fraser, A.G., Kamath, R.S., Ahringer, J., and Ruvkun, G. (2003). A systematic RNAi screen identifies a critical role for mitochondria in C. elegans longevity. 
Nat. Genet. 33, 40-48.

Li, H., and Durbin, R. (2009). Fast and accurate short read alignment with Burrows-Wheeler transform. Bioinformatics 25, 1754-1760.

Lin, J.-R., Sin-Chan, P., Napolioni, V., Torres, G.G., Mitra, J., Zhang, Q., Jabalameli, M.R., Wang, Z., Nguyen, N., and Gao, T. (2021). Rare genetic coding variants associated with human longevity and protection against age-related diseases. Nat. Aging 1,783-794.

Link, J.C., and Reue, K. (2017). Genetic basis for sex differences in obesity and lipid metabolism. Annu. Rev. Nutr. 37, 225-245.

Lopez-Otin, C., Blasco, M.A., Partridge, L., Serrano, M., and Kroemer, G. (2013). The hallmarks of aging. Cell 153, 1194-1217.

López-Otín, C., Blasco, M.A., Partridge, L., Serrano, M., and Kroemer, G. (2013). The hallmarks of aging. Cell 153, 1194-1217.

Lundquist, M.R., Goncalves, M.D., Loughran, R.M., Possik, E., Vijayaraghavan, T., Yang, A., Pauli, C., Ravi, A., Verma, A., and Yang, Z. (2018). Phosphatidylinositol-5-phosphate 4kinases regulate cellular lipid metabolism by facilitating autophagy. Mol. Cell 70, 531-544.

Mackay, G.M., Zheng, L., Van Den Broek, N.J.F., and Gottlieb, E. (2015). Analysis of cell metabolism using LC-MS and isotope tracers. Methods Enzymol. 561, 171-196.

Mair, W., Morantte, I., Rodrigues, A.P.C., Manning, G., Montminy, M., Shaw, R.J., and Dillin, A. (2011). Lifespan extension induced by AMPK and calcineurin is mediated by CRTC-1 and CREB. Nature 470, 404-408.

Martin, M. (2011). Cutadapt removes adapter sequences from high-throughput sequencing reads. EMBnet. J. 17, 10-12.

Matecic, M., Smith Jr, D.L., Pan, X., Maqani, N., Bekiranov, S., Boeke, J.D., and Smith, J.S. (2010). A microarray-based genetic screen for yeast chronological aging factors. PLoS Genet 6, e1000921.

Mauvais-Jarvis, F. (2015). Sex differences in metabolic homeostasis, diabetes, and obesity. Biol. Sex Differ. 6, 1-9.

Mauvais-Jarvis, F., Arnold, A.P., and Reue, K. (2017). A guide for the design of pre-clinical studies on sex differences in metabolism. Cell Metab. 25, 1216-1230.

McCarthy, D.J., Chen, Y., and Smyth, G.K. (2012). Differential expression analysis of multifactor RNA-Seq experiments with respect to biological variation. Nucleic Acids Res. 40, 4288-4297.

Meister, A. (1988). Glutathione metabolism and its selective modification. J. Biol. Chem. 263, 17205-17208.

Milinkovitch, T., Lefrancois, C., Durollet, M., and Thomas-Guyon, H. (2018). Influence of temperature on age-related lipid peroxidation in a short-lived vertebrate (Nothobranchius furzeri). Fish Physiol Biochem 44, 343-347.

Mohapatra, S., Chakraborty, T., Shimizu, S., Ohta, K., Nagahama, Y., and Ohta, K. (2020). Estrogen and estrogen receptors chauffeur the sex-biased autophagic action in liver. Cell Death Differ. 27, 3117-3130.

Murray, A.W. (1971). The biological significance of purine salvage. Annu. Rev. Biochem. $40,811-826$. 
Naqvi, S., Godfrey, A.K., Hughes, J.F., Goodheart, M.L., Mitchell, R.N., and Page, D.C. (2019). Conservation, acquisition, and functional impact of sex-biased gene expression in mammals. Science (80-. ). 365.

Niccoli, T., and Partridge, L. (2012). Ageing as a risk factor for disease. Curr Biol 22, R74152.

Ou, S., Su, W., Liao, Y., Chougule, K., Agda, J.R.A., Hellinga, A.J., Lugo, C.S.B., Elliott, T.A., Ware, D., and Peterson, T. (2019). Benchmarking transposable element annotation methods for creation of a streamlined, comprehensive pipeline. Genome Biol. 20, 1-18.

Pagès, H., Carlson, M., Falcon, S., and Li, N. (2021). AnnotationDbi: Manipulation of SQLite-based annotations in Bioconductor. R package version1. 54.1.

Palavicini, J.P., and Han, X. (2021). Lipidomics of aging. In Handbook of the Biology of Aging, (Elsevier), pp. 391-404.

Parafati, M., Kirby, R.J., Khorasanizadeh, S., Rastinejad, F., and Malany, S. (2018). A nonalcoholic fatty liver disease model in human induced pluripotent stem cell-derived hepatocytes, created by endoplasmic reticulum stress-induced steatosis. Dis. Model. Mech. $11, \mathrm{dmm} 033530$.

Patro, R., Duggal, G., Love, M.I., Irizarry, R.A., and Kingsford, C. (2017). Salmon provides fast and bias-aware quantification of transcript expression. Nat. Methods 14, 417-419.

Pernicova, I., and Korbonits, M. (2014). Metformin-mode of action and clinical implications for diabetes and cancer. Nat. Rev. Endocrinol. 10, 143-156.

Pietzke, M., and Vazquez, A. (2020). Metabolite AutoPlotter-an application to process and visualise metabolite data in the web browser. Cancer Metab. 8, 1-11.

Porath, H.T., Carmi, S., and Levanon, E.Y. (2014). A genome-wide map of hyper-edited RNA reveals numerous new sites. Nat. Commun. 5, 1-10.

Porath, H.T., Knisbacher, B.A., Eisenberg, E., and Levanon, E.Y. (2017). Massive A-to-I RNA editing is common across the Metazoa and correlates with dsRNA abundance. Genome Biol. 18, 1-12.

Pradas, I., Jové, M., Huynh, K., Puig, J., Inglés, M., Borrás, C., Viña, J., Meikle, P.J., and Pamplona, R. (2019). Exceptional human longevity is associated with a specific plasma phenotype of ether lipids. Redox Biol. 21, 101127.

Reichwald, K., Petzold, A., Koch, P., Downie, B.R., Hartmann, N., Pietsch, S., Baumgart, M., Chalopin, D., Felder, M., Bens, M., et al. (2015). Insights into Sex Chromosome Evolution and Aging from the Genome of a Short-Lived Fish. Cell 163, 1527-1538.

Robinson, M.D., and Oshlack, A. (2010). A scaling normalization method for differential expression analysis of RNA-seq data. Genome Biol. 11, 1-9.

Robinson, M.D., McCarthy, D.J., and Smyth, G.K. (2010). edgeR: a Bioconductor package for differential expression analysis of digital gene expression data. Bioinformatics 26, 139 140 .

Roth, S.H., Levanon, E.Y., and Eisenberg, E. (2019). Genome-wide quantification of ADAR adenosine-to-inosine RNA editing activity. Nat. Methods 16, 1131-1138.

Sahota, A., Ranjekar, P.K., Alfonzo, J., Lewin, A.S., and Taylor, M.W. (1987). Mutants of Saccharomyces cerevisiae deficient in adenine phosphoribosyltransferase. Mutat. Res. Mol. Mech. Mutagen. 180, 81-87. 
Schmieder, R., and Edwards, R. (2011). Quality control and preprocessing of metagenomic datasets. Bioinformatics 27, 863-864.

Semba, R.D., Zhang, P., Adelnia, F., Sun, K., Gonzalez-Freire, M., Salem Jr, N., Brennan, N., Spencer, R.G., Fishbein, K., and Khadeer, M. (2019). Low plasma lysophosphatidylcholines are associated with impaired mitochondrial oxidative capacity in adults in the Baltimore Longitudinal Study of Aging. Aging Cell 18, e12915.

Sharma, S., Wang, J., Alqassim, E., Portwood, S., Gomez, E.C., Maguire, O., Basse, P.H., Wang, E.S., Segal, B.H., and Baysal, B.E. (2019). Mitochondrial hypoxic stress induces widespread RNA editing by APOBEC3G in natural killer cells. Genome Biol. 20, 1-17.

Sharples, A.P., Hughes, D.C., Deane, C.S., Saini, A., Selman, C., and Stewart, C.E. (2015). Longevity and skeletal muscle mass: the role of IGF signalling, the sirtuins, dietary restriction and protein intake. Aging Cell 14, 511-523.

Smith, H.J., Sharma, A., and Mair, W.B. (2020). Metabolic communication and healthy aging: Where should we focus our energy? Dev. Cell.

Stenesen, D., Suh, J.M., Seo, J., Yu, K., Lee, K.-S., Kim, J.-S., Min, K.-J., and Graff, J.M. (2013). Adenosine nucleotide biosynthesis and AMPK regulate adult life span and mediate the longevity benefit of caloric restriction in flies. Cell Metab. 17, 101-112.

Sun, L.Y., Fang, Y., Patki, A., Koopman, J.J.E., Allison, D.B., Hill, C.M., Masternak, M.M., Darcy, J., Wang, J., and McFadden, S. (2017). Longevity is impacted by growth hormone action during early postnatal period. Elife 6, e24059.

Sun, N., Youle, R.J., and Finkel, T. (2016). The mitochondrial basis of aging. Mol. Cell 61, 654-666.

Tacutu, R., Thornton, D., Johnson, E., Budovsky, A., Barardo, D., Craig, T., Diana, E., Lehmann, G., Toren, D., Wang, J., et al. (2018). Human Ageing Genomic Resources: new and updated databases. Nucleic Acids Res 46, D1083-D1090.

Tavallaie, M., Voshtani, R., Deng, X., Qiao, Y., Jiang, F., Collman, J.P., and Fu, L. (2020). Moderation of mitochondrial respiration mitigates metabolic syndrome of aging. Proc. Natl. Acad. Sci. 117, 9840-9850.

Terzibasi, E., Lefrançois, C., Domenici, P., Hartmann, N., Graf, M., and Cellerino, A. (2009). Effects of dietary restriction on mortality and age-related phenotypes in the short-lived fish Nothobranchius furzeri. Aging Cell.

Tramunt, B., Smati, S., Grandgeorge, N., Lenfant, F., Arnal, J.-F., Montagner, A., and Gourdy, P. (2020). Sex differences in metabolic regulation and diabetes susceptibility. Diabetologia 63, 453-461.

Ulgherait, M., Rana, A., Rera, M., Graniel, J., and Walker, D.W. (2014). AMPK modulates tissue and organismal aging in a non-cell-autonomous manner. Cell Rep. 8, 1767-1780.

Valenzano, D.R., Terzibasi, E., Genade, T., Cattaneo, A., Domenici, L., and Cellerino, A. (2006). Resveratrol prolongs lifespan and retards the onset of age-related markers in a shortlived vertebrate. Curr. Biol.

Valenzano, D.R., Benayoun, B.A., Singh, P.P., Zhang, E., Etter, P.D., Hu, C.K., ClementZiza, M., Willemsen, D., Cui, R., Harel, I., et al. (2015). The African Turquoise Killifish Genome Provides Insights into Evolution and Genetic Architecture of Lifespan. Cell 163, 1539-1554.

Valle, A., Guevara, R., Garcia-Palmer, F.J., Roca, P., and Oliver, J. (2007). Sexual 
dimorphism in liver mitochondrial oxidative capacity is conserved under caloric restriction conditions. Am. J. Physiol. Physiol. 293, C1302-C1308.

Ventura-Clapier, R., Moulin, M., Piquereau, J., Lemaire, C., Mericskay, M., Veksler, V., and Garnier, A. (2017). Mitochondria: a central target for sex differences in pathologies. Clin. Sci. 131, 803-822.

Wang, Y., and Hekimi, S. (2015). Mitochondrial dysfunction and longevity in animals: untangling the knot. Science (80-. ). 350, 1204-1207.

Wiatrek, D.M., Candela, M.E., Sedmík, J., Oppelt, J., Keegan, L.P., and O’Connell, M.A. (2019). Activation of innate immunity by mitochondrial dsRNA in mouse cells lacking p53 protein. Rna 25, 713-726.

Woods, A., Williams, J.R., Muckett, P.J., Mayer, F. V, Liljevald, M., Bohlooly-Y, M., and Carling, D. (2017). Liver-specific activation of AMPK prevents steatosis on a high-fructose diet. Cell Rep. 18, 3043-3051.

Wu, B.N., and O'Sullivan, A.J. (2011). Sex differences in energy metabolism need to be considered with lifestyle modifications in humans. J. Nutr. Metab. 2011.

Yang, X., Mudgett, J., Bou-About, G., Champy, M.-F., Jacobs, H., Monassier, L., Pavlovic, G., Sorg, T., Herault, Y., and Petit-Demoulière, B. (2016). Physiological expression of AMPK $\gamma 2$ RG mutation causes Wolff-Parkinson-White syndrome and induces kidney injury in mice. J. Biol. Chem. 291, 23428-23439.

Yavari, A., Stocker, C.J., Ghaffari, S., Wargent, E.T., Steeples, V., Czibik, G., Pinter, K., Bellahcene, M., Woods, A., and De Morentin, P.B.M. (2016). Chronic activation of $\gamma 2$ AMPK induces obesity and reduces $\beta$ cell function. Cell Metab. 23, 821-836.

Yee, C., Yang, W., and Hekimi, S. (2014). The intrinsic apoptosis pathway mediates the prolongevity response to mitochondrial ROS in C. elegans. Cell 157, 897-909.

Young, G., Lin, J., Cheng, Y., Ho, C., Kuok, Q., Hsu, R., Liao, W., Chen, C., and Chen, H. (2021). Modulation of adenine phosphoribosyltransferase-mediated salvage pathway to accelerate diabetic wound healing. FASEB J. 35, e21296.

Yu, G., Wang, L.-G., Han, Y., and He, Q.-Y. (2012). clusterProfiler: an R package for comparing biological themes among gene clusters. Omi. a J. Integr. Biol. 16, 284-287.

Zhou, Z., Moore, T.M., Drew, B.G., Ribas, V., Wanagat, J., Civelek, M., Segawa, M., Wolf, D.M., Norheim, F., and Seldin, M.M. (2020). Estrogen receptor $\alpha$ controls metabolism in white and brown adipocytes by regulating Polg1 and mitochondrial remodeling. Sci. Transl. Med. 12. 
Figure 1 bioRxiv preprint doi: https://doi.org/10.1101/2022.01.10.475524; this version posted January 10, 2022. The copyright holder for this preprint (which was not certified by peer review) is the author/funder. All rights reserved. No reuse allowed without permission.

A. ATP production via the nucleotide

B. Generation of APRT CRISPR mutant salvage pathway
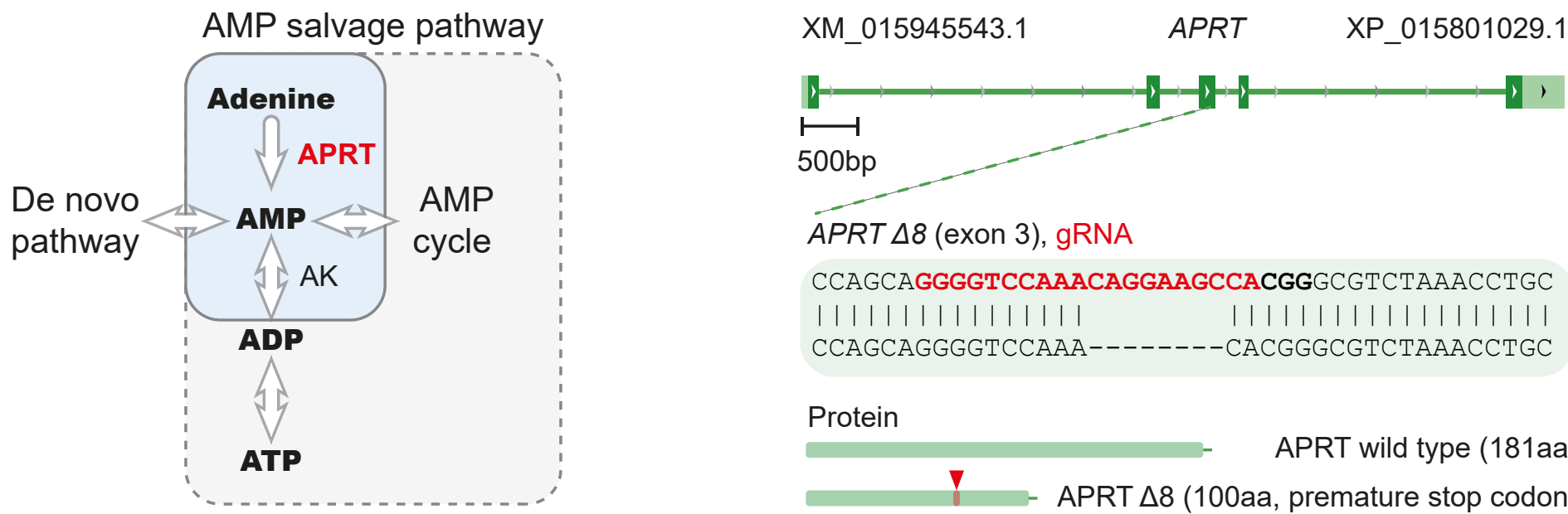

Protein

APRT wild type (181aa) $\nabla$ - APRT $\Delta 8$ (100aa, premature stop codon)

C. Lifespan of $A P R T^{\triangle 8 /+}$ males

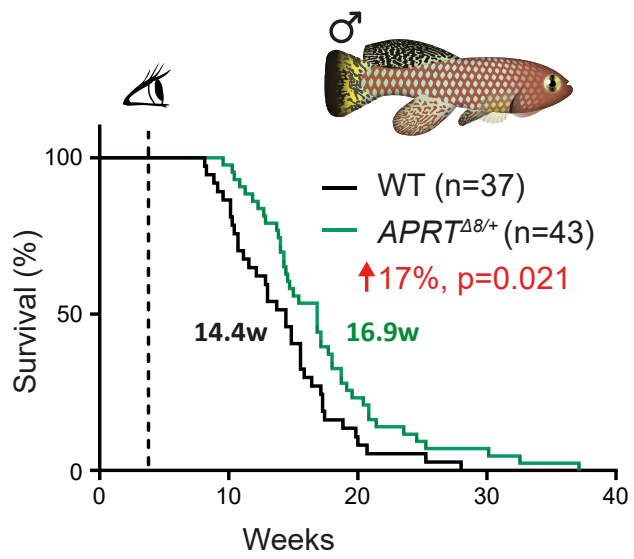

D. Lifespan of $A P R T^{\Delta 8 /+}$ females

E. Male and female lifespan

F. Experimental design

Sample collection

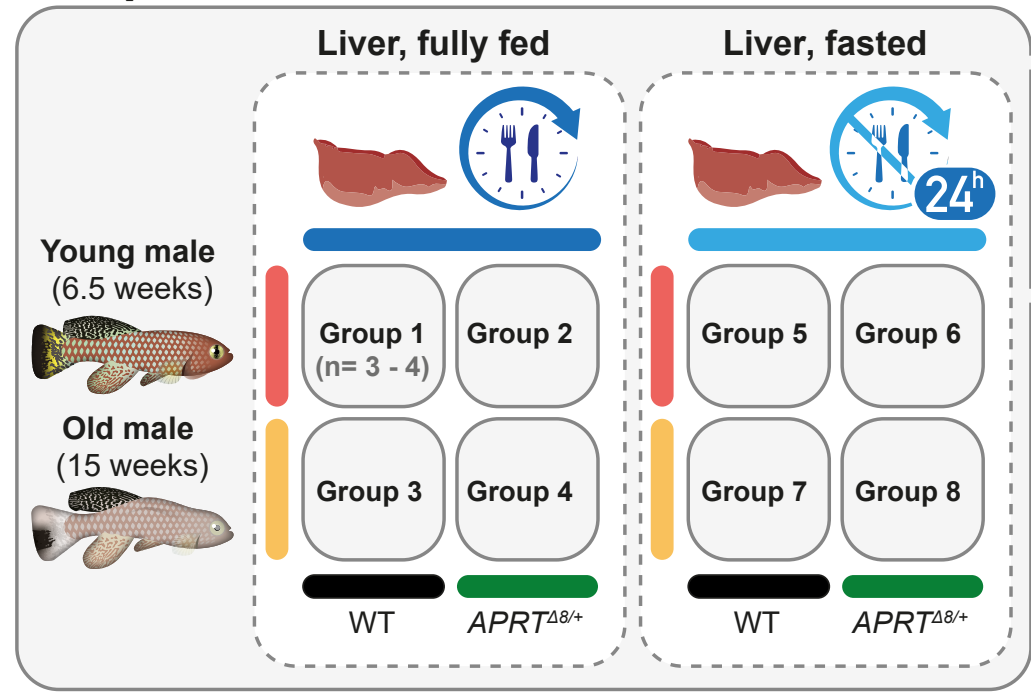

운

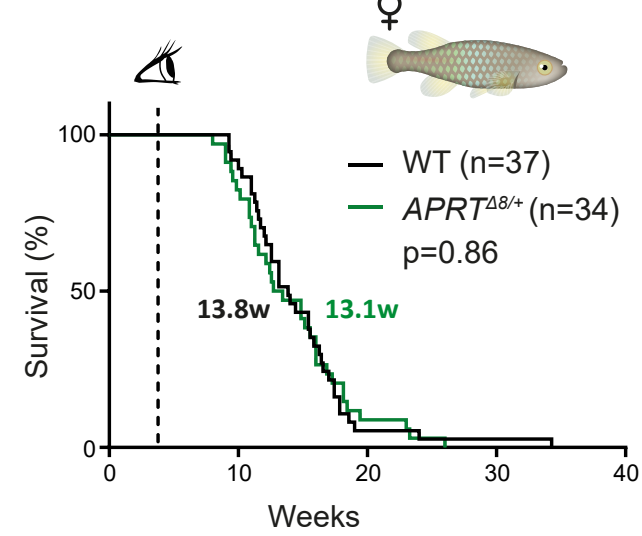

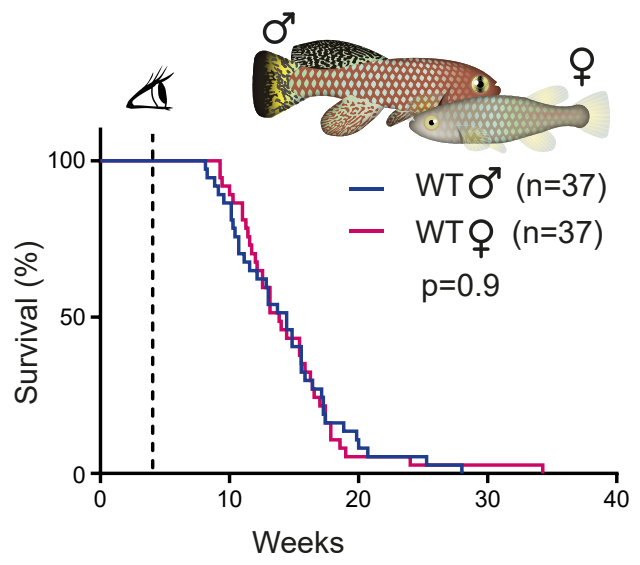

RNA sequencing
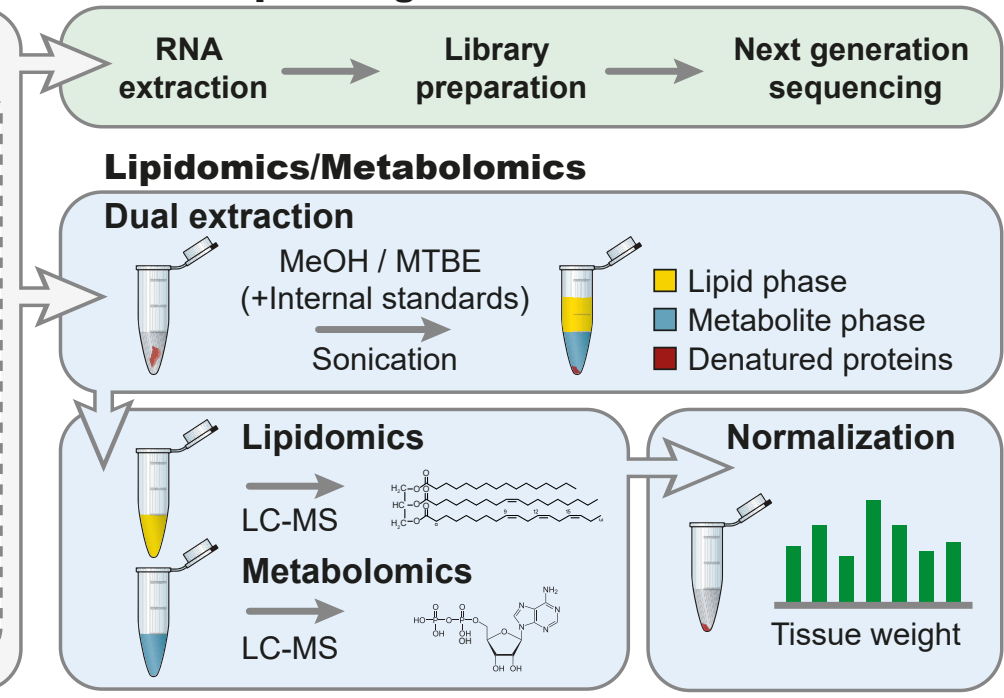

Lipidomics Normalization

Metabolomics

LC-MS 
Figure 1: Male-specific lifespan extension by targeting APRT, a key enzyme in the AMP biosynthesis

(A) As a part of the nucleotide salvage pathway, the enzyme adenine phosphoribosyltransferase (APRT) catalyzes Adenine into AMP, a key precursor for ATP production. AMP can be also produced by other pathways, including the AMP de novo pathway and the AMP cycle. Adenylate kinase (AK) enzymes catalyze the reversible conversion of adenine nucleotides $(2 \mathrm{ADP}=\mathrm{ATP}+\mathrm{AMP})$ to generate ATP

(B) Generation of APRT CRISPR mutant, including guide RNA (gRNA) target for exon 3 (red sequence), protospacer adjacent motif (or PAM, in bold), and successful germline transmission of an $8 \mathrm{bp}$ deletion $(\Delta 8)$. The $A P R T \Delta 8$ allele is predicted to generate a protein with a premature stop codon.

(C-E) Lifespan of WT and $A P R T^{\Delta 8 /+}$ fish assessed separately for males (C) and females (D). (E) Overlay of the male and female lifespans from WT fish, using data from (C) and (D). p values for differential survival in log-rank tests, median survival, and fish numbers are indicated. See Table S1 for complete statistics.

(F) Analysis pipeline for characterizing the livers of male fish using RNA sequencing, metabolomics, and lipidomics. Experimental groups differ by age (young or old), genotype (WT or $A P R T^{\Delta 8 /+}$ ), and feeding condition (fully-fed or fasted for $24 \mathrm{~h}$ ). 
Figure 2 bioRxiv preprint doi: https://doi.org/10.1101/2022.01.10.475524; this version posted January 10, 2022. The copyright holder for this preprint (which was not certified by peer review) is the author/funder. All rights reserved. No reuse allowed without permission.

A. PCA for transcript levels under different feeding conditions
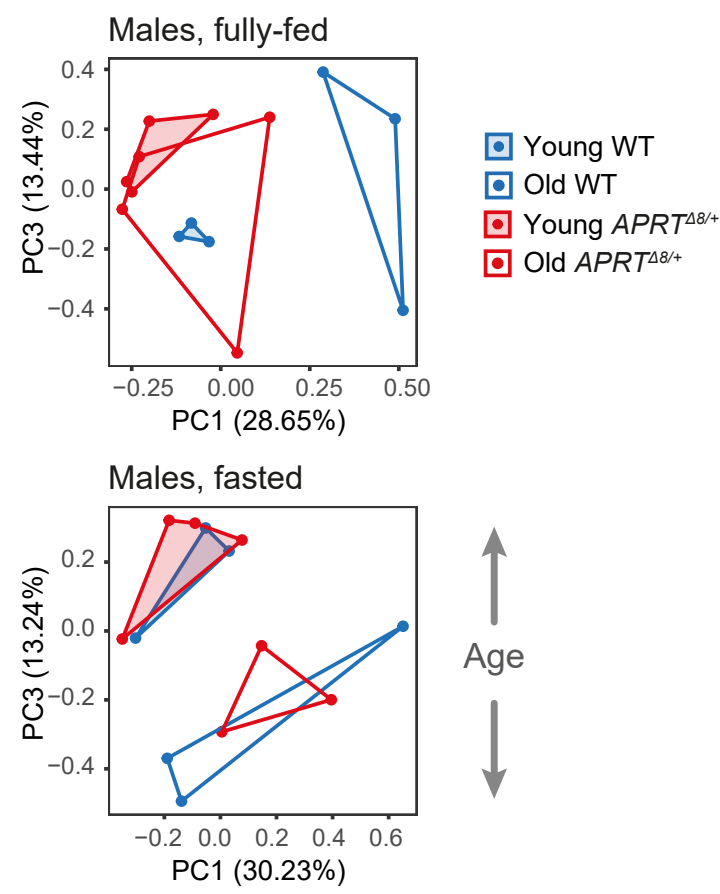

C. Pathways enriched across male liver samples during aging

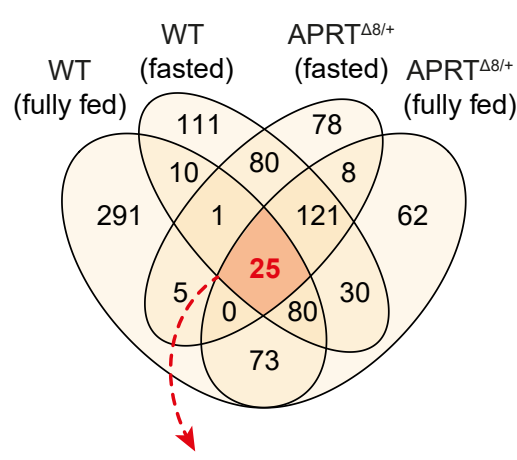

Inflammatory processes (24/25)

Activation of immune response

$T$ cell receptor signaling pathway

Antigen processing and presentation

Defense response to bacterium

Others (1/25)

Anatomical structure homeostasis
B. GSEA for the aging liver recapitulates the hallmarks of aging
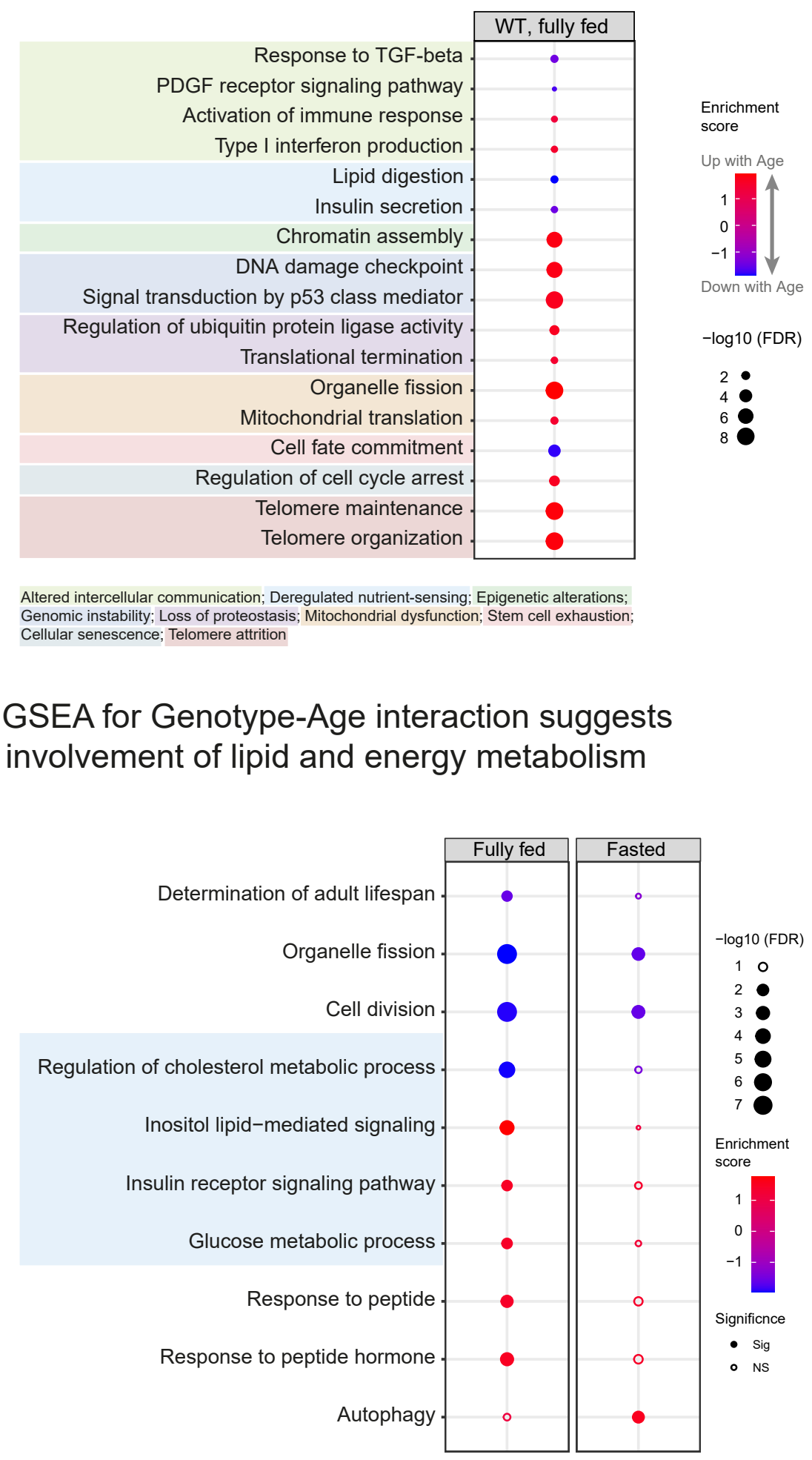

Enrichment
score

Up with Age

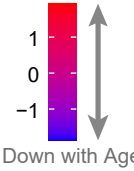

$-\log 10$ (FDR)

\section{GSEA for Genotype-Age interaction suggests involvement of lipid and energy metabolism}

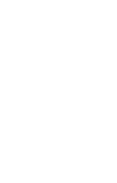


Figure 2: Transcriptomic analysis in males suggests a modified energy and lipid metabolism in the livers of old $A P R T^{\Delta 8 /+}$ fish

(A) Principal component analysis (PCA) for transcript levels from the livers of WT (blue) or $A P R T^{\Delta 8 /+}$ (red) fish, either young (shaded) or old (nonshaded). Experiments were performed under fully-fed (top) or fasting conditions (bottom). Each symbol represents an individual fish. PC1/PC3 are shown. PC1/PC2 are reported in Figure S2.

(B) Functional enrichments (GO) using Gene Set Enrichment Analysis (GSEA) for differential RNA expression with aging (WT, fully-fed). Enrichment significance was at FDR $<5 \%$.

(C) Venn diagram showing the overlap of significantly enriched pathways with aging across all male samples. GO Enrichments using GSEA were called at FDR $<5 \%$.

(D) Genotype-Age interaction analysis to identify enriched pathways (using GSEA) that codepend on both genotype and age (e.g. how the $A P R T^{\Delta 8 /+}$ mutation modifies the aging process). Analysis was performed separately for fully-fed or fasting conditions. GO Enrichments using GSEA were called at FDR $<5 \%$. 
Figure 3 bioRxiv preprint doi: https://doi.org/10.1101/2022.01.10.475524; this version posted January 10, 2022. The copyright holder for this preprint (which was not certified by peer review) is the author/funder. All rights reserved. No reuse allowed without permission.

A. PCA for metabolite levels under different feeding conditions
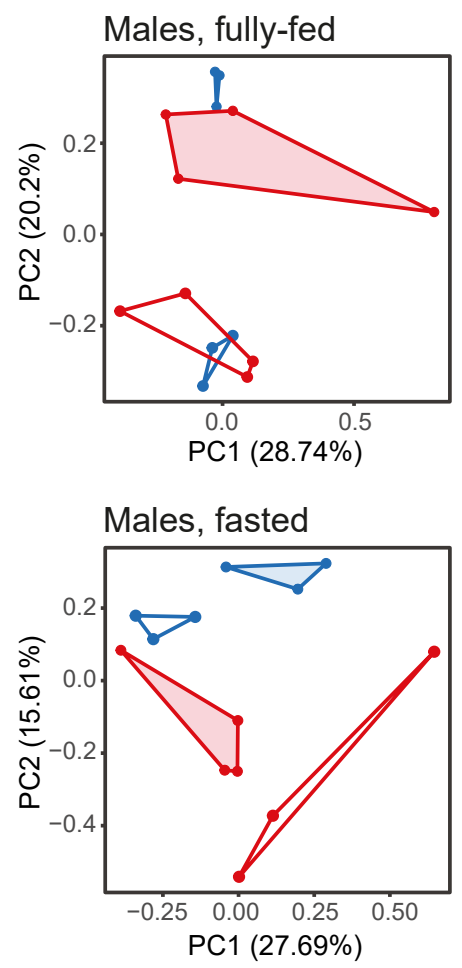

- Young WT Young APRT ${ }^{\Delta 8 /+}$

Old WT $\square$ Old APRT

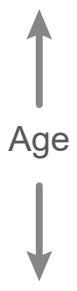

Genotype

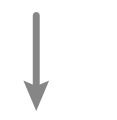

B. Old WT fish display a deregulated nutrient sensing signature in response to fasting, which is rejuvenated in $A P R T^{\Delta 8 /+}$ fish

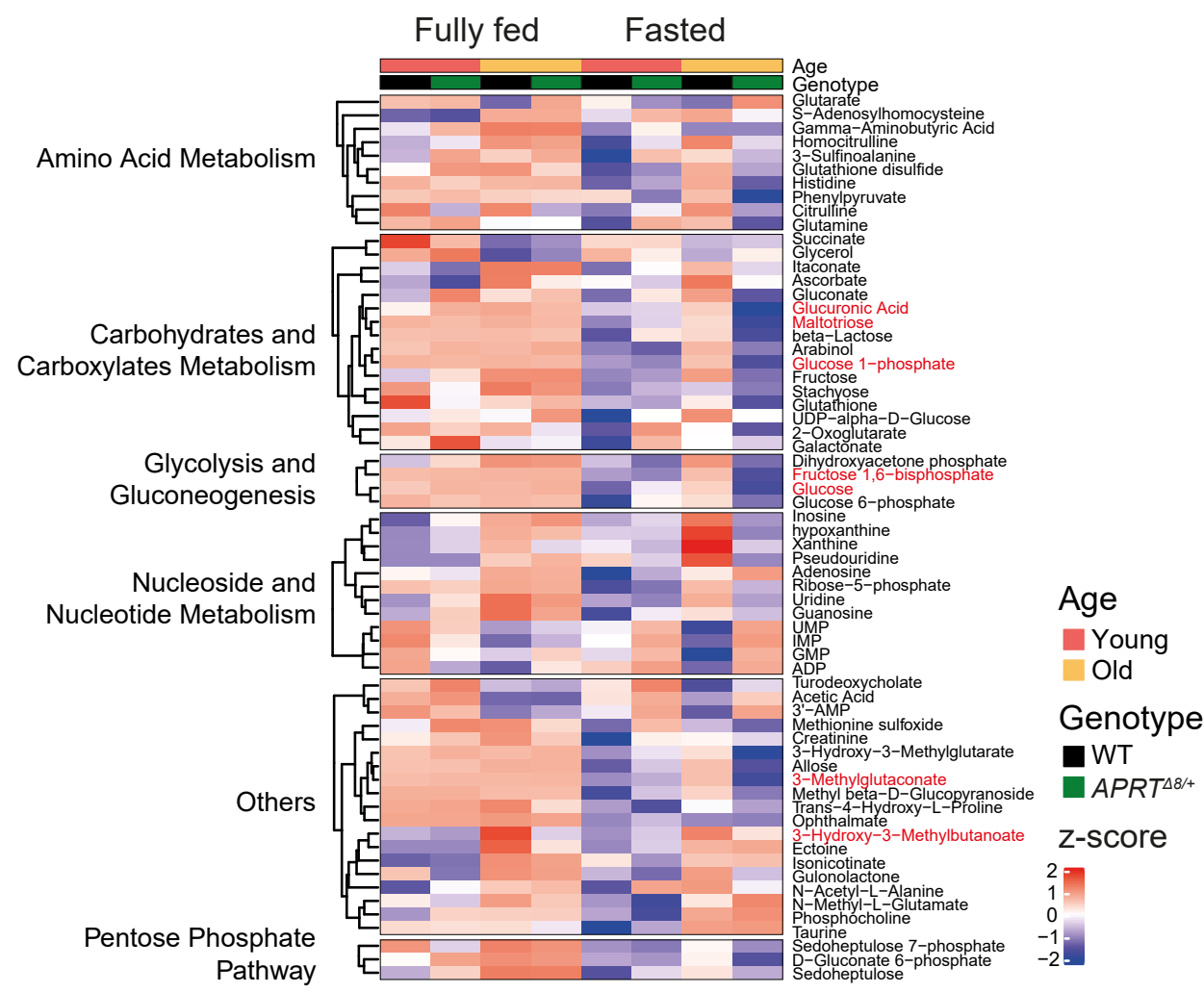

C. Selected metabolites in fasted old WT fish significantly cluster with fully fed conditions

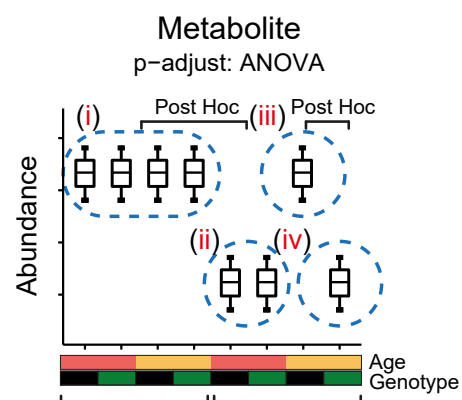

Fully fed Fasted

Fully fed Fasted

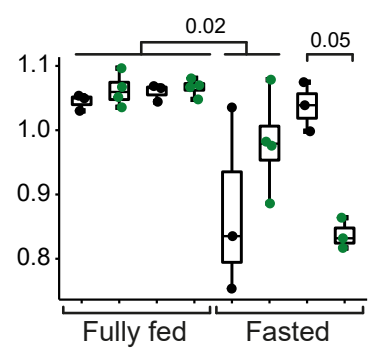

Glucose 1-phosphate ANOVA: $4.3 \times 10^{5}$

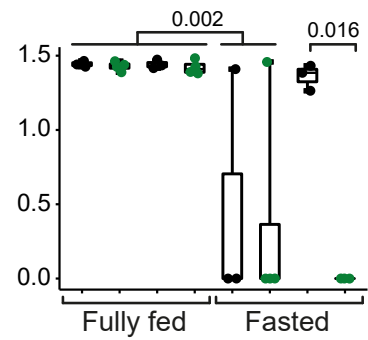

Fructose 1,6-bisphosphate ANOVA: $4.3 \times 10^{5}$

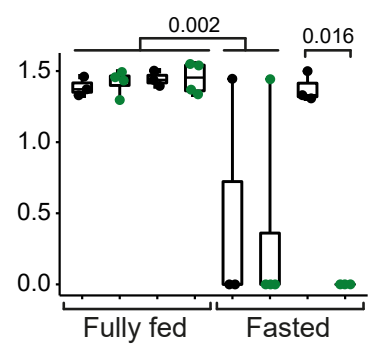

Age

Young

Old

Genotype

- WT

$A P R T^{\triangle 8 /+}$
ATP synthesis

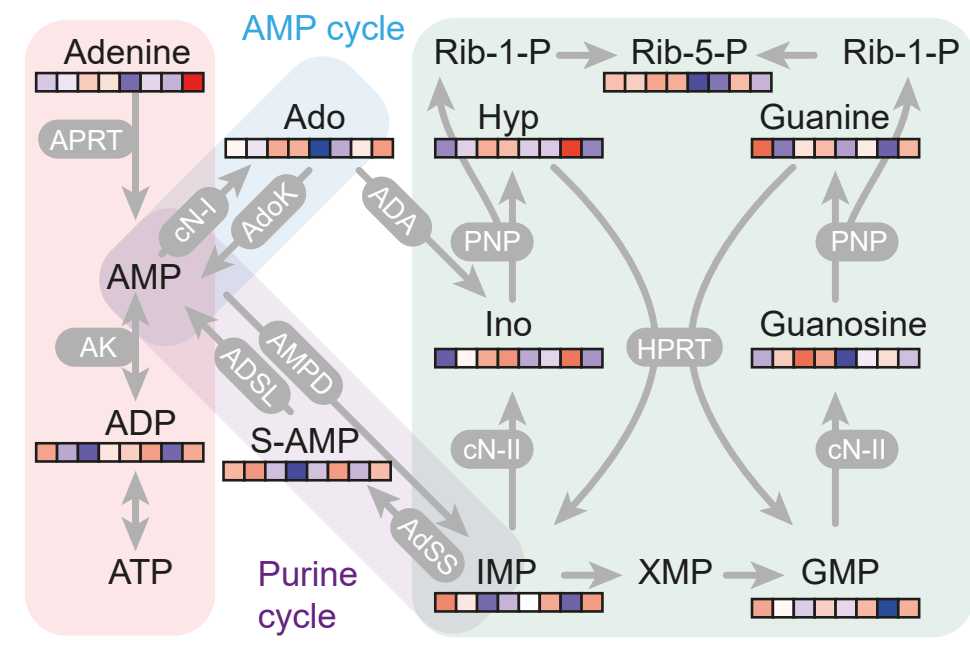

IMP-GMP cycle
Glycolysis

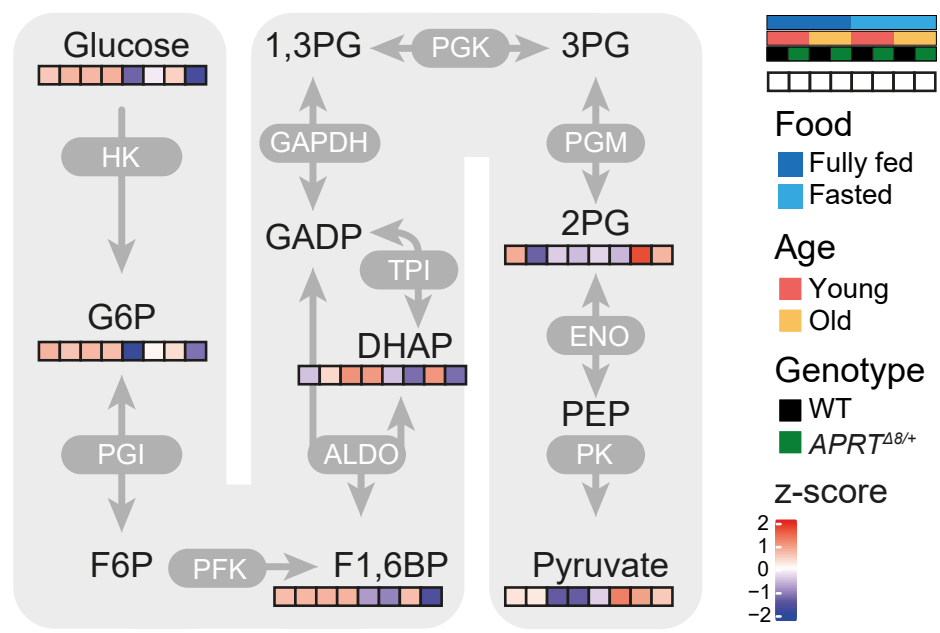




\section{Figure 3: Metabolomic analysis demonstrates restoration of energy homeostasis in livers of old $A P R T^{48 /+}$ fish}

(A) Principal component analysis (PCA) for metabolite levels from the livers of WT (blue) or $A P R T^{\Delta 8 /+}$ (red) fish, young (shaded) or old (nonshaded). Experiments were performed under fully-fed (top) or fasting conditions (bottom). Each symbol represents an individual fish.

(B) Heatmap showing significantly altered liver metabolites clustered into key metabolic pathways (full metabolite list is reported in Figure S3 and Table S3). Each square represents the normalized relative abundance (indicated by color), from an average of 3-4 fish. Significant metabolites were evaluated by one-way ANOVA between 4 clusters as depicted in (C): fully fed (i); young fasting (ii); old WT fasting (iii); and old $A P R T^{\Delta 8 /+}$ fasting (iv). Using these clusters, we can estimate the normal response to fasting (comparing clusters $\mathbf{i}$ and $\mathbf{i i}$ ), and detect the age-dependent deregulated nutrient sensing that is rejuvenated in old $A P R T^{\Delta 8 /+}$ fish (comparing clusters iii and iv). Significance was called at FDR $\leq 5 \%$ for both ANOVA and Tukey post-hoc. The full statistical values are reported in Table S3.

(C) Boxplot showing significant metabolites selected from (B), of carbohydrate and glucose metabolism. $n=3-4$ fish per experimental condition, each symbol represents one fish. Bars represent minimum and maximum.

(D) Selected metabolic pathways depicting key enzymes and abundance levels of main compounds. Each square represents the color-coded normalized relative abundance as seen in (B). Pathways were curated from the KEGG (Kanehisa, 2017) and BioCyc (Caspi et al., 2020). 2PG: 2-Phospho-D-Glycerate; Ado: Adenosine; DHAP: Dihydroxyacetone phosphate; F1,6BP: D-Fructose 1,6-bisphosphate; G6P: Glucose 6-phosphate; Hyp: hypoxanthine; Ino: Inosine; Rib-5-P: Ribose-5-phosphate; S-AMP: Adenylo-Succinate; Rib-1-P: Ribose-1phosphate; F6P: Fructose 6-phosphate; 1,3PG: 1,3-Bisphosphoglycerate; GADP: Glyceraldehyde 3-phosphate; 3PG: 3-Phosphoglyceric acid; PEP: Phosphoenolpyruvate 
Figure 4 bioRxiv preprint doi: https://doi.org/10.1101/2022.01.10.475524; this version posted January 10, 2022. The copyright holder for this preprint (which was not certified by peer review) is the author/funder. All rights reserved. No reuse allowed without permission.

\section{A. Experimental design}

Sample collection (for RNA sequencing)

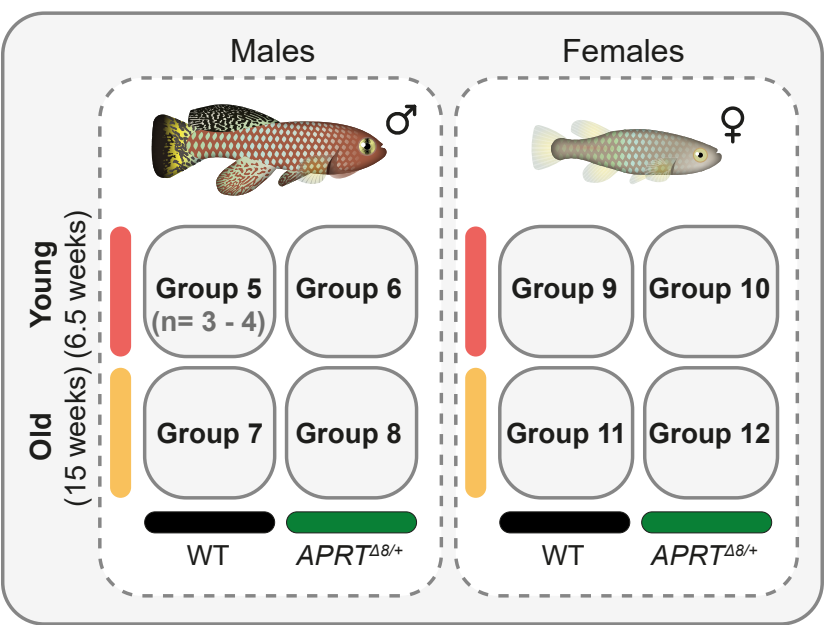

\section{Gene expression fold change between old and young fish (Old/Young)}

Fatty acid beta-oxidation

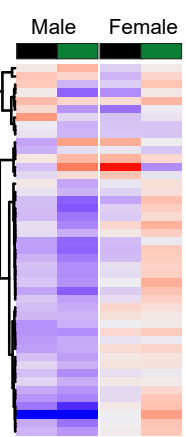

Negative regulation of organelle organization

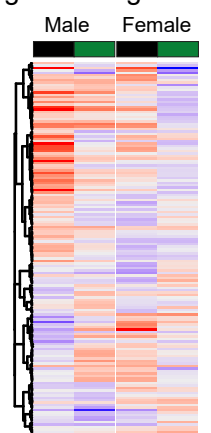

Regulation of steroid metabolic process

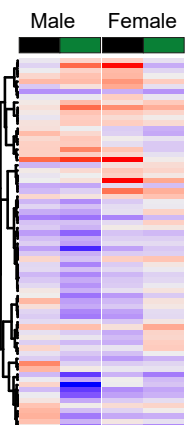

B. GSEA for the aging liver identifies sex related differences between old and young fish

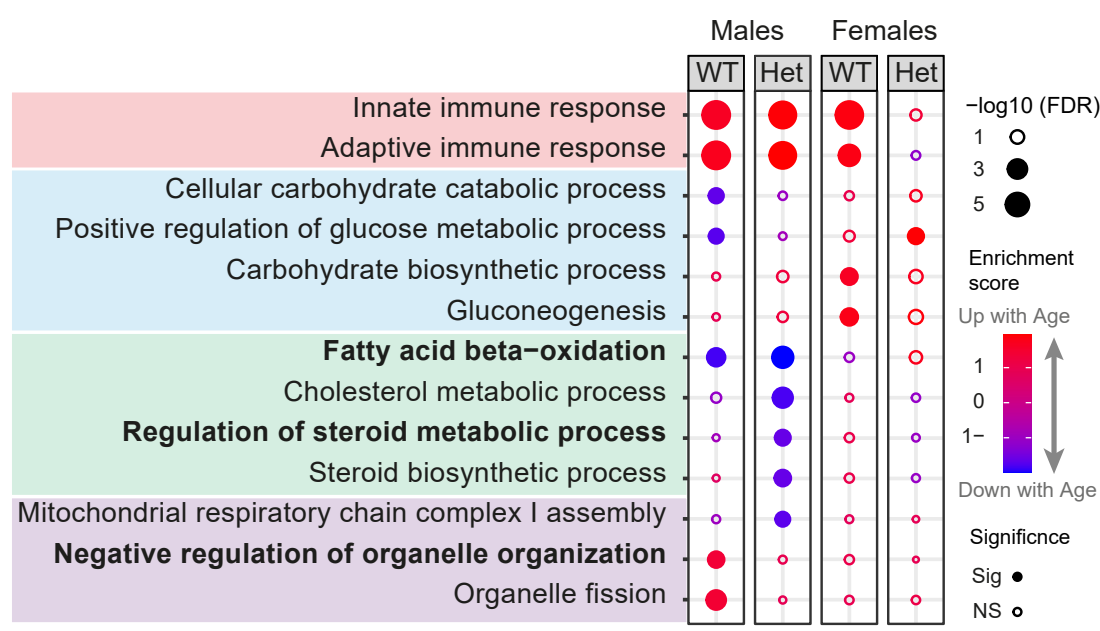

Genotype

$\log \mathrm{FC}$

Up with age

4

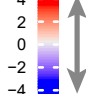

Down with age

\section{GSEA for Genotype-Age interaction highlights sex-specific mitochondrial functions}

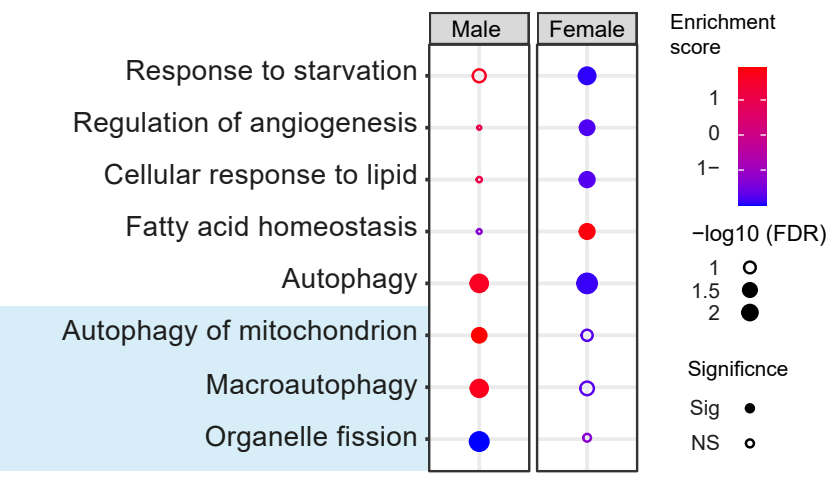




\section{Figure 4: The male-specific lifespan extension in $A P R T^{48 /+}$ fish is associated with a distinct mitochondrial transcriptional signature}

(A) Experimental design for characterizing the livers of male and female fish using RNA sequencing. Experimental groups differ by age (young or old) and genotype (WT or APRT ${ }^{\Delta 8 /+}$ ). Note that there is a single feeding condition in this design (i.e. $24 \mathrm{~h}$ fasting), and that male fish (experimental groups \#5-8) are also presented in Figure 1f.

(B) Functional enrichments (GO) using Gene Set Enrichment Analysis (GSEA) for differential RNA expression between old and young fish. A comparison between the different experimental groups demonstrates conserved (e.g. inflammatory processes, in red) and sex-specific dynamics. Sex-specific pathways include energy metabolism, specifically carbohydrate and lipid metabolism (in blue or green, respectively) and mitochondrial functions (in purple). Pathways in bold are also depicted in (C). GO enrichments using GSEA were called at FDR < $5 \%$.

(C) Heatmap for age-related gene expression changes in male and female livers (representative pathways are selected from $(\mathbf{B})$ ). Genes are hierarchically clustered, and expression values are presented as Log2 (fold-change) between old and young using a 1.5-fold change cutoff. Each column represents the fold change between old and young.

(D) Genotype-Age interaction analysis was used to identify sex-specific pathways that codepend on both genotype and age. Functional enrichments (GO) were performed using Gene Set Enrichment Analysis (GSEA). GO enrichments using GSEA were called at FDR < 5\%. 
Figure 5 bioRxiv preprint doi: https://doi.org/10.1101/2022.01.10.475524; this version posted January 10, 2022. The copyright holder for this preprint (which was not certified by peer review) is the author/funder. All rights reserved. No reuse allowed without permission.

A. Mitochondrial DNA copy number is increased in old $A P R T^{\Delta 8 /+}$ male livers

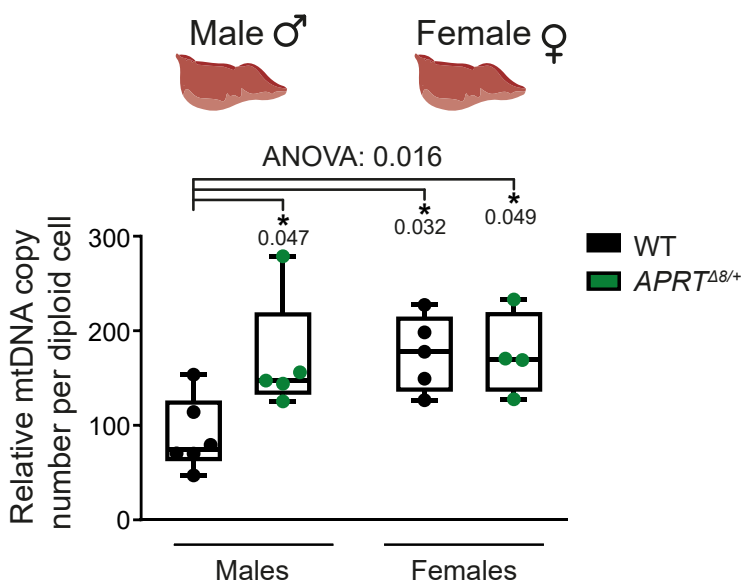

B. Mitochondrial DNA copy number is increased in tails of old $A P R T^{\Delta 8 /+}$ males

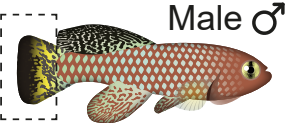

Female 우

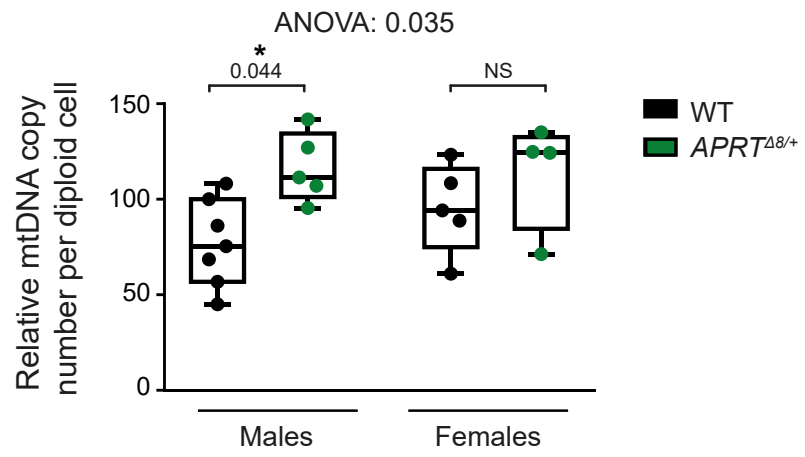

C. Generation of fibroblast primary cell culture from the tail fin of wild-type (WT) and $A P R T^{\triangle 8 /+}$ males

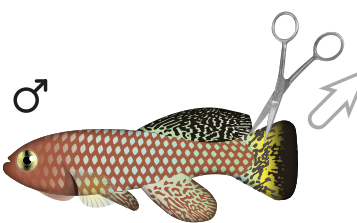

Individual fish as biological replicates
Tail fin dissection

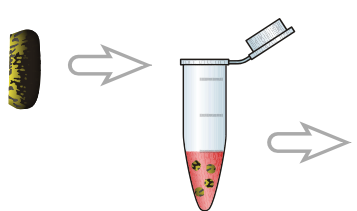

Mechanical dissociation

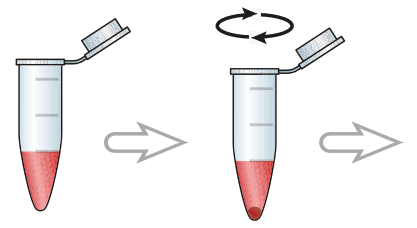

Enzymatic digestion
Centrifugation

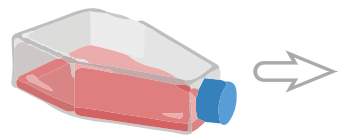

Plating and expansion of cells

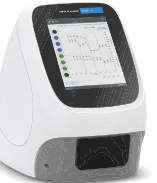

Downstream assays (e.g. cell biology, Seahorse)
D. Reduced proliferation in $A P R T^{\Delta 8 /+}$-derived cells

ANOVA: 0.0016

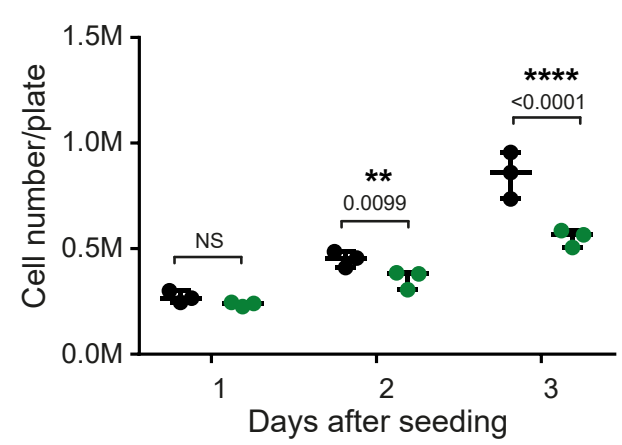

E. $A P R T^{\Delta 8 /+}$-derived cells are partially resistant to 8-Azaadenine

ANOVA: 0.0009

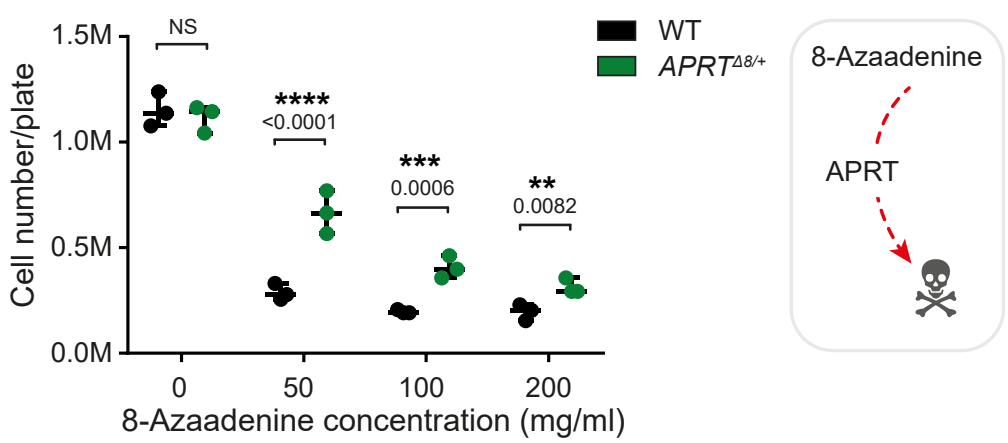




\section{Figure 5: Mitochondrial DNA copy numbers are sex- and genotype-dependent}

(A-B) Relative mitochondrial DNA (mtDNA) copy number in old fish livers (A) or tails (B) was determined by quantitative real-time PCR (qPCR) using primers for mitochondrial (16S $r R N A)$ and nuclear $(C d k n 2 a / b)$ gene. Experimental groups differ by sex (male or female) and genotype (WT or $A P R T^{\Delta 8 /+}$ ). Each symbol represents an individual fish. Bars represent minimum and maximum, and significance was measured by one-way ANOVA and Sidak's multiple comparisons test. Exact p-values are indicated.

(C) Schematic illustration depicting the isolation procedure of primary fibroblasts from the tail-fin of individual fish. Isolations and downstream assays were separately performed on WT and $A P R T^{\Delta 8 /+}$ male fish ( $\mathrm{n}=3$ for each genotype).

(D-E) Assessing cell proliferation rates (D) and resistance to 8-Azaadenine (E) in WT- and $A P R T^{\Delta 8 /+}$-derived primary cells under the indicated experimental conditions. Each symbol represents a cell culture derived from an individual fish. Bars represent minimum and maximum, significance was measured by two-way ANOVA, and BKY (Benjamini, Krieger, $\&$ Yekutieli, false discovery rate) correction for multiple tests. 
Figure 6 bioRxiv preprint doi: https://doi.org/10.1101/2022.01.10.475524; this version posted January 10, 2022. The copyright holder for this preprint (which was not certified by peer review) is the author/funder. All rights reserved. No reuse allowed without permission.

A. Mitochondrial respiration profile ('Mito Stress' assay)

Oligomycin FCCP Rotenone \&

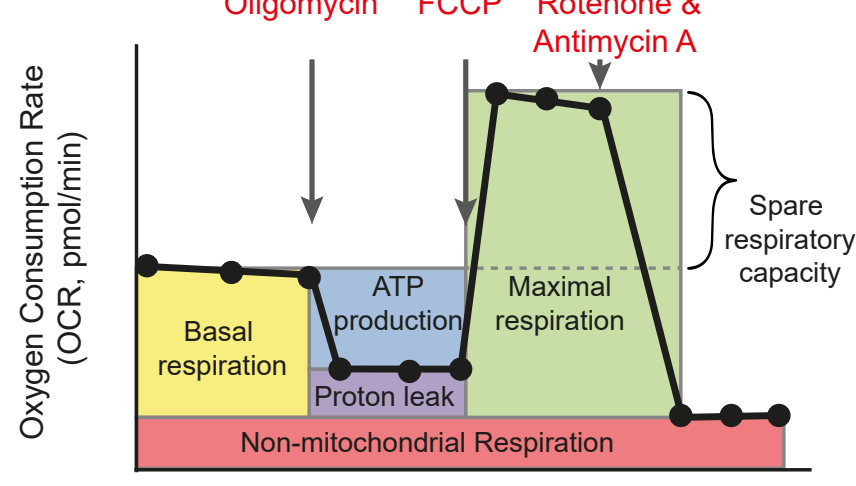

Time (minutes)
B. Reduced mitochondrial respiration in $A P R T^{\triangle 8 /+}$-derived cells

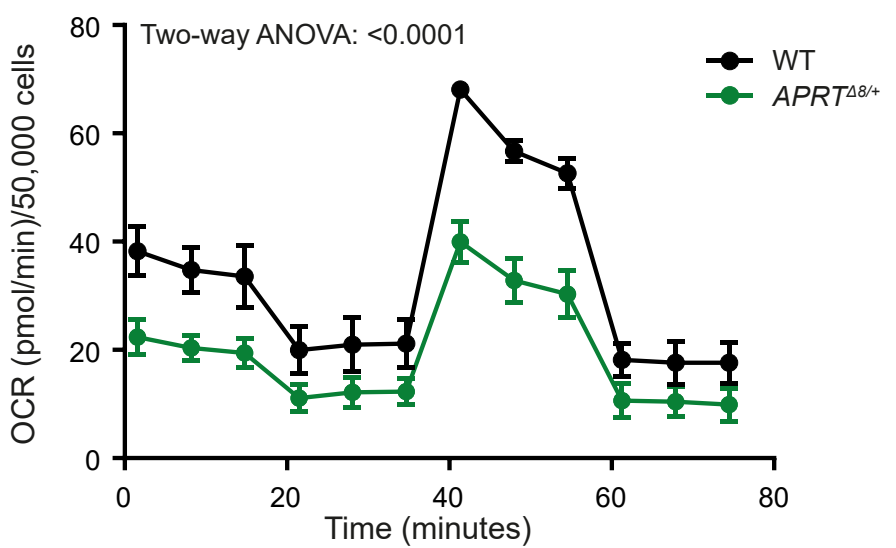

C. Mitochondrial respiration is partially rescued by Adenine

WT + Adenine - $A P R T^{\Delta 8 /+}+$ Adenine

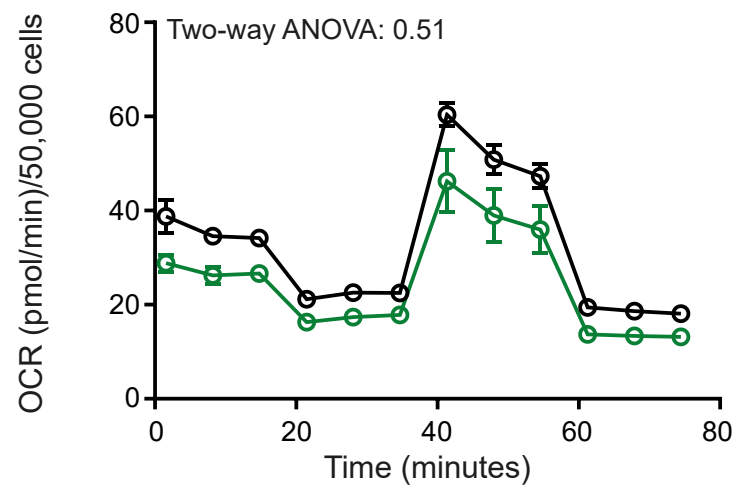

D. Mitochondrial basal respiration and ATP production

- WT O WT + Adenine

- $A P R T^{\Delta 8 /+} \bigcirc A P R T^{\Delta 8 /+}+$ Adenine

Basal respiration

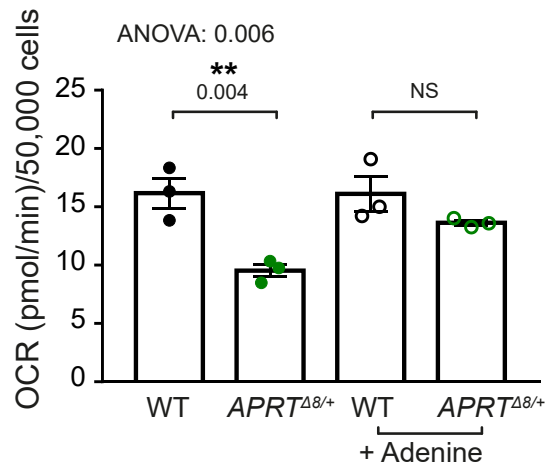

ATP production

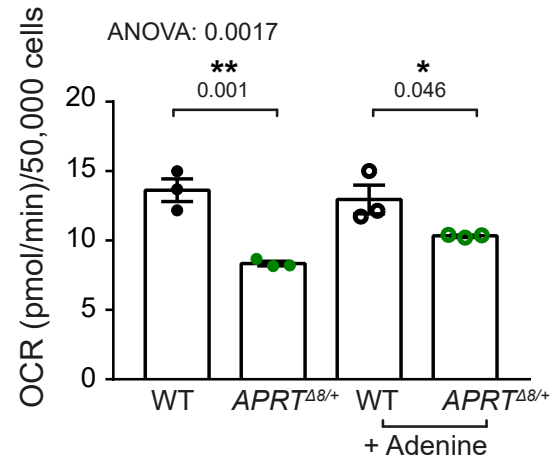

E. Mitochondrial morphology is altered in APRT ${ }^{\triangle 8 /+}$-derived cells
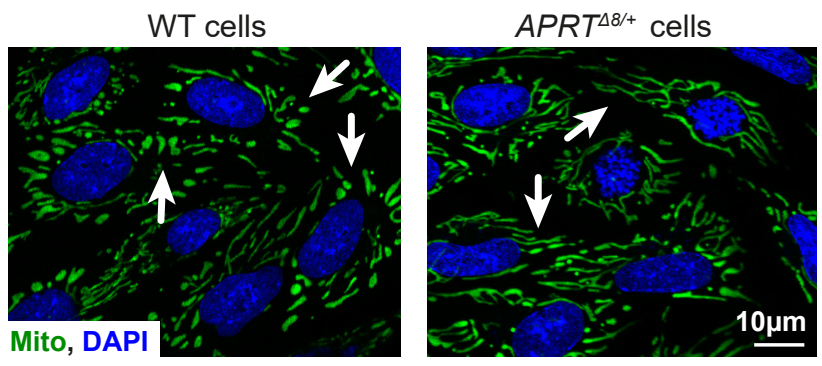

Mitochondria footprint

Branch mean length
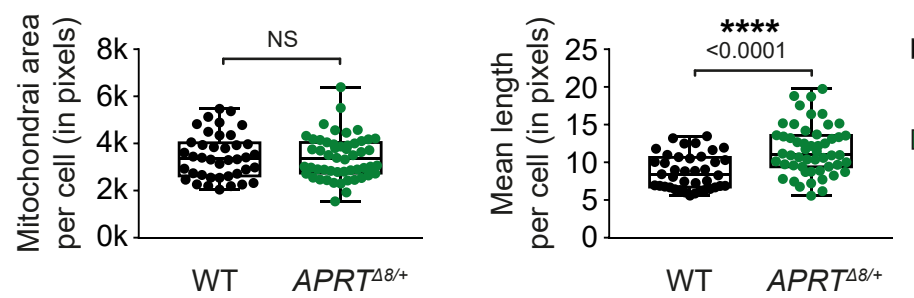

WT $(n=40)$

$A P R T^{\Delta 8 /+}$ $(\mathrm{n}=52)$ 


\section{Figure 6: altered mitochondrial functions in $A P R T^{\Delta 8 /+}$-derived cells}

(A) Measurement of key mitochondrial parameters as a function of Oxygen Consumption Rate (OCR, pmol/min) using the Seahorse Cell Mito Stress Test assay (Agilent). Measurements were performed under basal conditions or following the addition of the indicated inhibitors. Specific parameters, such as the rates of basal respiration and ATP production, were further normalization by actual cell numbers $(\mathbf{D})$.

(B-C) Measuring mitochondrial respiration in WT- and $A P R T^{\Delta 8 /+}$-derived primary cells. Experiments were performed in control conditions (B) and Adenine supplementation (C). Each symbol represents an average of three independent measurements using cells derived from three individual fish. Error bars represent mean \pm SEM, and significance was measured by twoway ANOVA and BKY correction.

(D) Basal respiration and ATP production were compared between WT- and $A P R T^{\triangle 8 /+}$-derived primary cells for control (B) and adenine supplementation (C). Each symbol represents an individual fish. Error bars represent mean \pm SEM, and significance was measured one-way ANOVA and Tukey post-hoc.

(E) Left: live imaging of WT- and $A P R T^{\Delta 8 /+}$-derived primary cells using mitochondrial staining

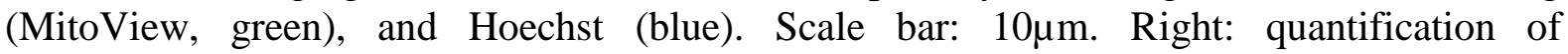
mitochondrial footprint (volume) and branch mean length was performed using MiNA macro plugin (Fiji - ImageJ). Data were obtained from 3 independent animals per genotype, with the indicated number of individualized mitochondria. Error bars represent mean \pm SEM and the significance were measured by an unpaired t-test. 
Fiqure 7 bioRxiv preprint doi: https://doi.org/10.1101/2022.01.10.475524; this version posted January 10, 2022. The copyright holder for this preprint (which was not certified by peer review) is the author/funder. All rights reserved. No reuse allowed without permission.

A. AMPK is activated in heterozygous $A P R T^{\triangle 8 /{ }_{-}}$ derived cells, mimicking fasting conditions

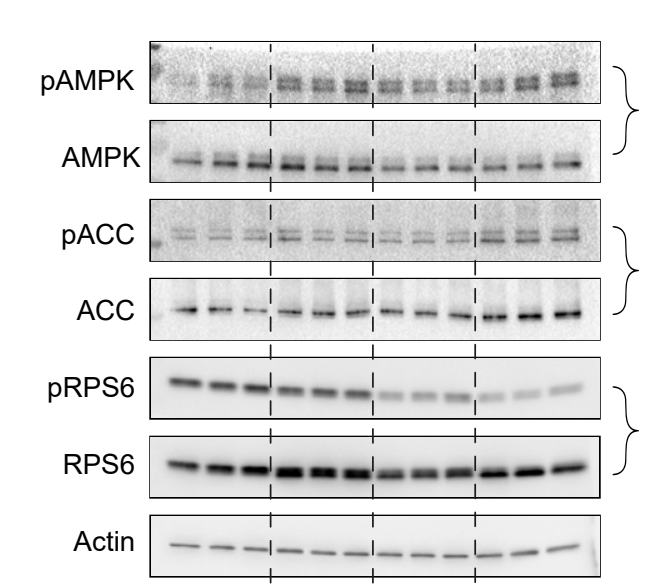

Genotype

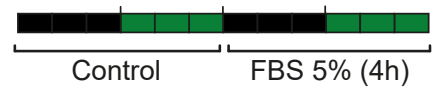

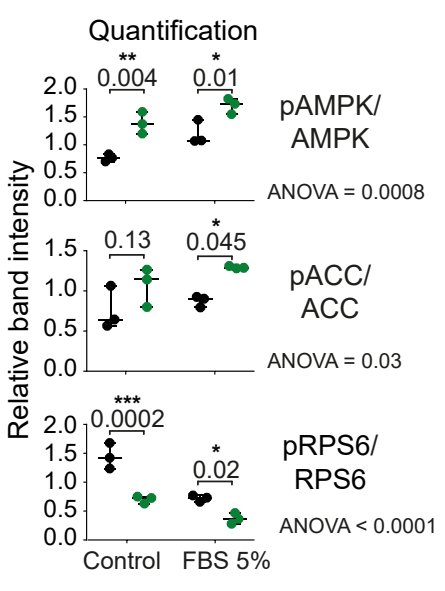

WT $\square A P R T^{\Delta 8 /+}$
B. Altered nucleotide pool in $A P R T^{\Delta 8 /+}$-derived cells

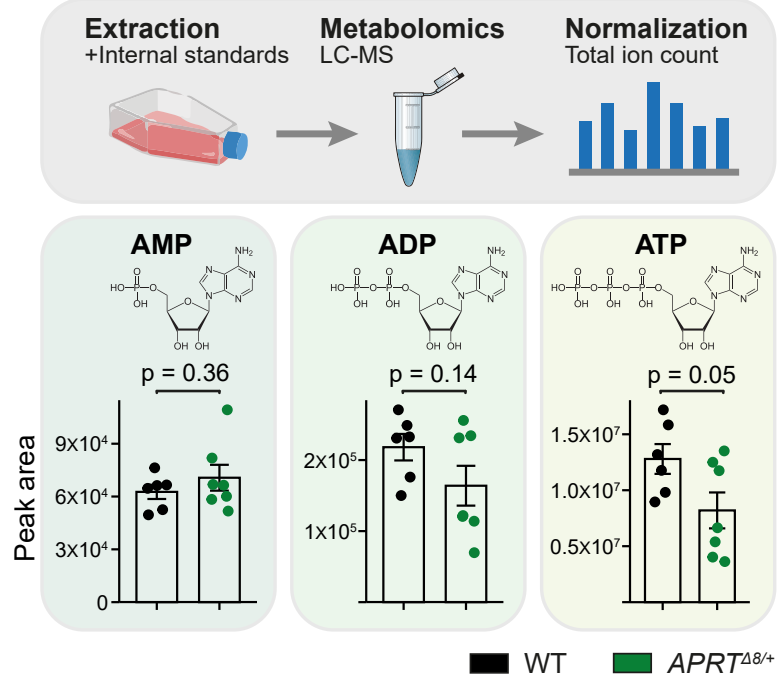

C. Old $A P R T^{\Delta 8 /+}$ male fish are resistant to high-fat diet

High-fat diet, males
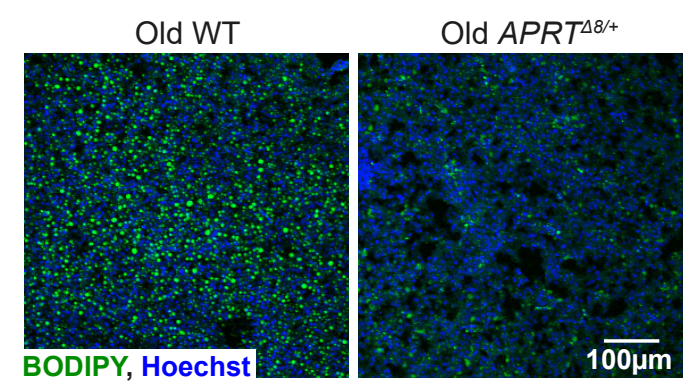

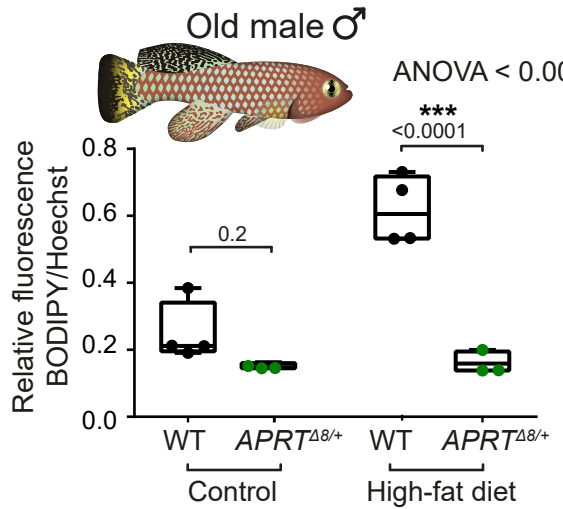

E. Model

D. Intermittent fasting rescues the male-specific longevity effects of the $A P R T^{\Delta 8 /+}$ mutation
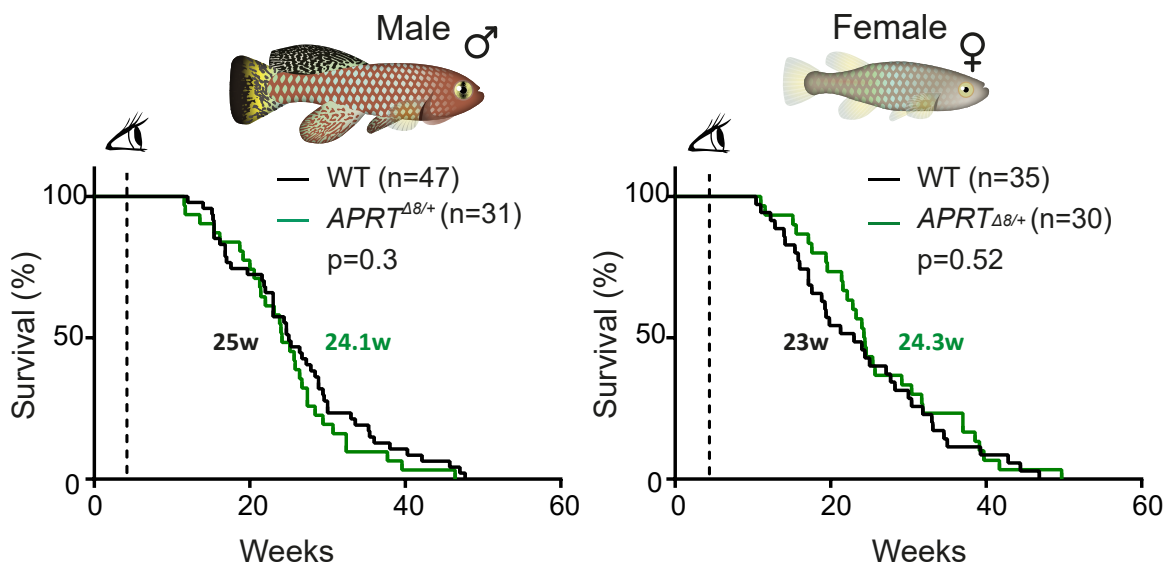
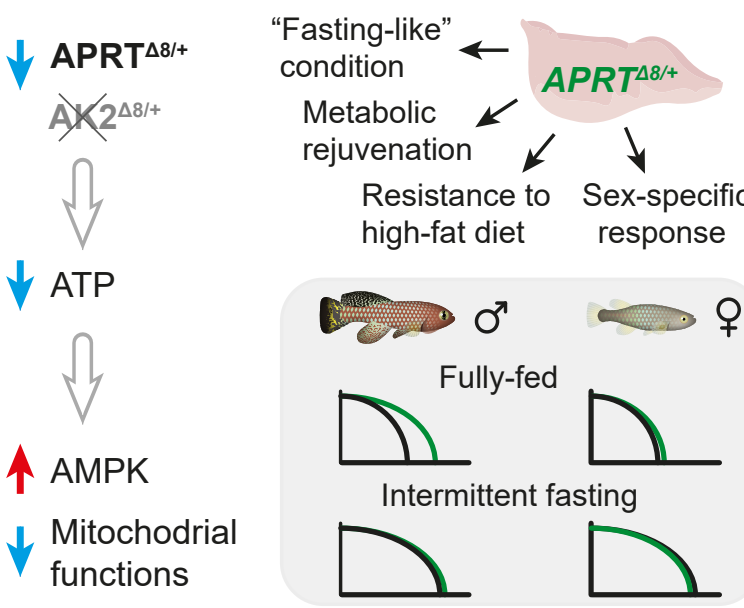

Old female $\mathrm{q}$

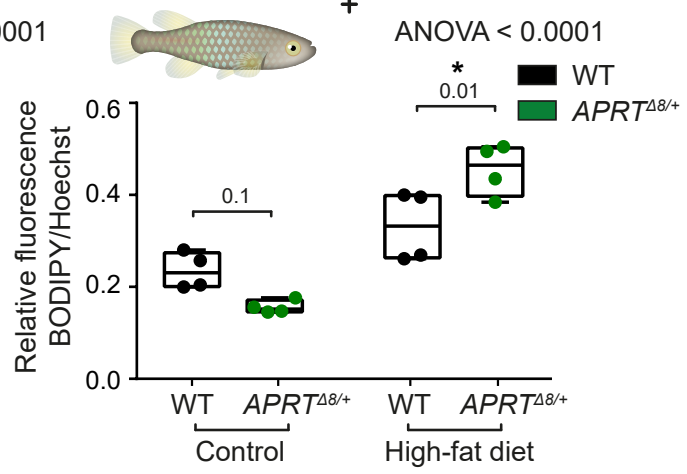




\section{Figure 7: A sex-specific fasting-like state mediates the longevity effect of the APRT mutation}

(A) Western blot (left) of primary metabolic pathways in WT- and $A P R T^{\Delta 8 /+}$-derived primary cells. Quantification (Fiji - ImageJ, right) represents the ratio between the phosphorylated and non-phosphorylated forms. Experiments were performed under either control or fasting (5\% FBS, $4 \mathrm{~h}$ ) conditions. Data from 3 biological replicates for each experimental condition. Bars represent minimum and maximum, significance was measured by one-way ANOVA and Sidak's multiple comparisons test. Exact p-values are indicated. AMPK: AMPK $\alpha$; pAMPK: Phospho-AMPKa (Thr172); ACC: Acetyl-CoA Carboxylase; pACC: Phospho-Acetyl-CoA Carboxylase (Ser79); RPS6: S6 Ribosomal Protein; pRPS6: Phospho-S6 Ribosomal Protein (Ser235/236).

(B) Direct measurement of the nucleotide pool (relative concentration of AMP, ADP, and ATP) in WT- and $A P R T^{\Delta 8 /+}$-derived primary cells using liquid chromatography-mass spectrometry (LC-MS). Data were obtained from 6-7 biological replicates for each experimental condition, and normalized by total ion count (TIC). Bars represent mean \pm SEM, unpaired t-test and exact p-values are indicated.

(C) Hepatic lipid accumulation and quantification in response to high-fat diet. Left: BODIPY staining (green) of liver section. Nuclei were stained by Hoechst (blue). Scale bar represents $100 \mu \mathrm{m}$. Right: Quantification of the relative fluorescence intensity of BODIPY/Hoechst (FijiImageJ). Experimental groups differ by sex (males or females), feeding condition (control or high-fat diet), and genotype (WT or $A P R T^{\Delta 8 /+}$ ). Bars represent minimum and maximum, and significance was measured by one-way ANOVA and Sidak's multiple comparisons test. Exact p-values are indicated.

(D) Lifespan of WT (black) and $A P R T^{\Delta 8 /+}$ (green) fish performed separately for males (left) and females (right) under intermittent fasting conditions. $p$ values for differential survival in log-rank tests, median survival, and fish numbers are presented. See Table S1 for complete statistics.

(G) A model suggesting that APRT, a member of the AMP biosynthesis pathway, could function as a regulator for vertebrate longevity. Mutating APRT induces a sex-specific longevity effect, mediated by restoring metabolic homeostasis, modulating mitochondrial functions, and inducing a fasting-like state that involves a distinct AMPK signature. 
Figure S pioRxiv preprint doi: https://doi.org/10.1101/2022.01.10.475524; this version posted January 10, 2022. The copyright holder for this preprint (which was not certified by peer review) is the author/funder. All rights reserved. No reuse allowed without permission.

A. The $A P R T^{\triangle 8 / \Delta 8}$ homozygous mutation is $\sigma^{7}$ embryonic lethal

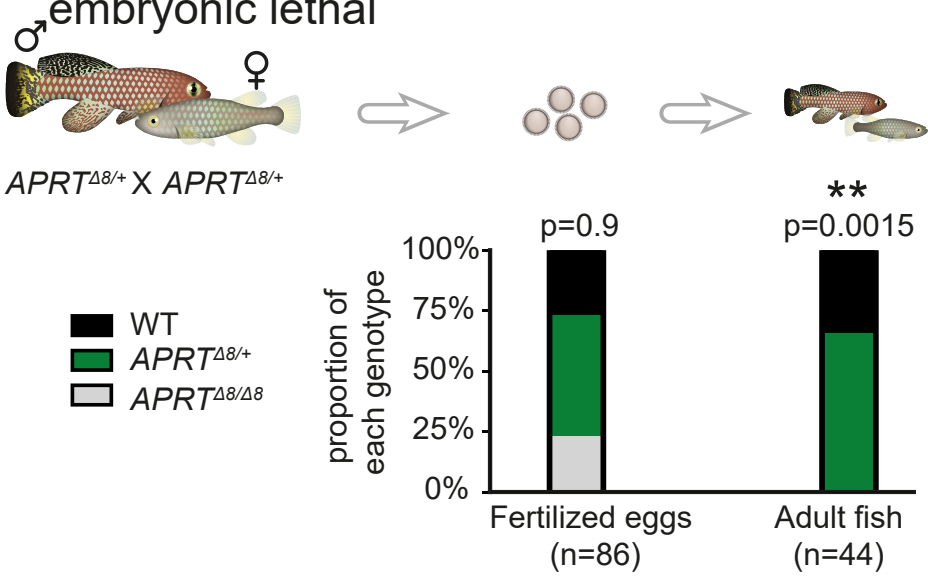

C. Egg size from $A P R T^{\triangle 8 /+}$ and WT mating pairs

WT pairs
APRT $^{\triangle 8 /+}$ pairs

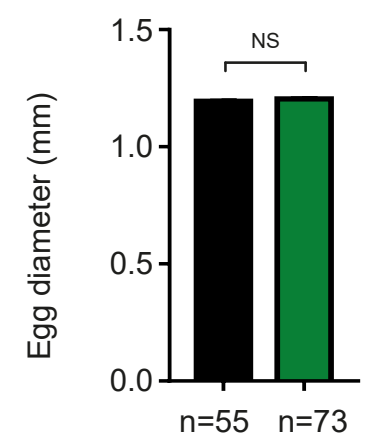

E. Generation of $A K 2$ knock-in CRISPR mutant

XM_015974135.1

XP_015829621.1

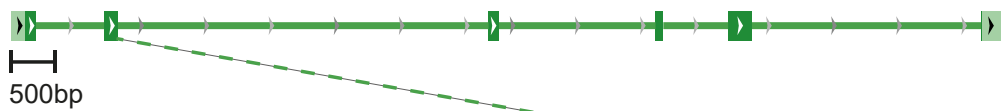

$A K 2 \Delta 8$, and knock-in of Nhel (exon 2), gRNA

CCTGGCCACCGGAGACATGC--TGAGGGCCATGGTGGCATCCGGC----TCC--GAACTTG

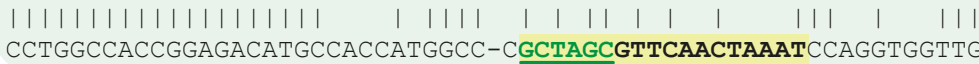

Protein

AK2 wild type (206aa)

AK2 $\Delta 8$, Nhel knock-in (104aa, premature stop codon)

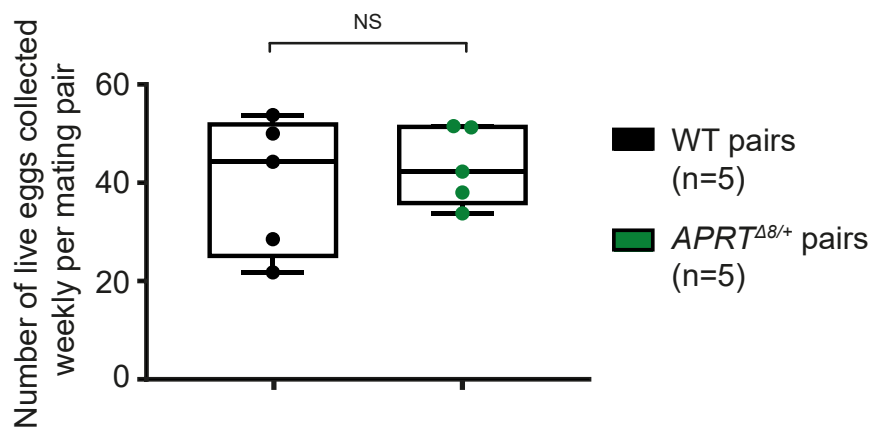

D. A phylogenetic tree for AK2

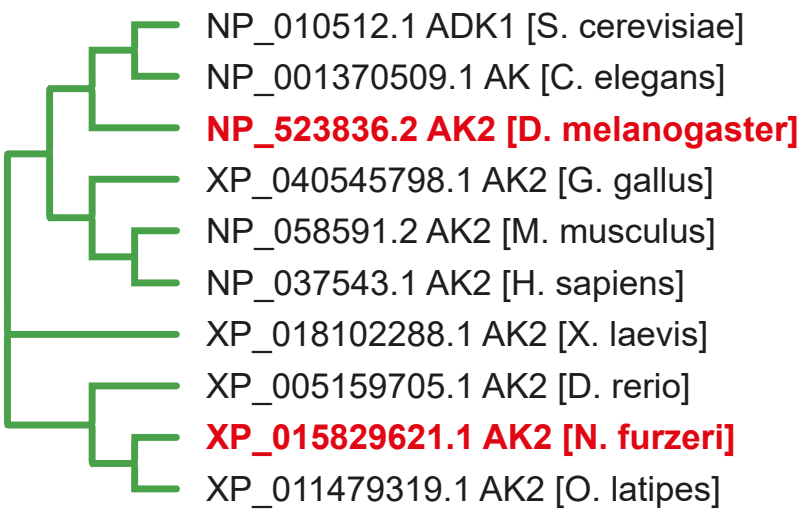

F. The $A K 2^{\Delta 8 / \Delta 8}$ mutation is embryonic lethal

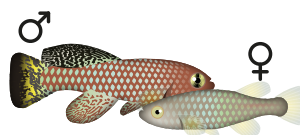

$A K 2^{\triangle 8 /+} \times A K 2^{\triangle 8 /+}$
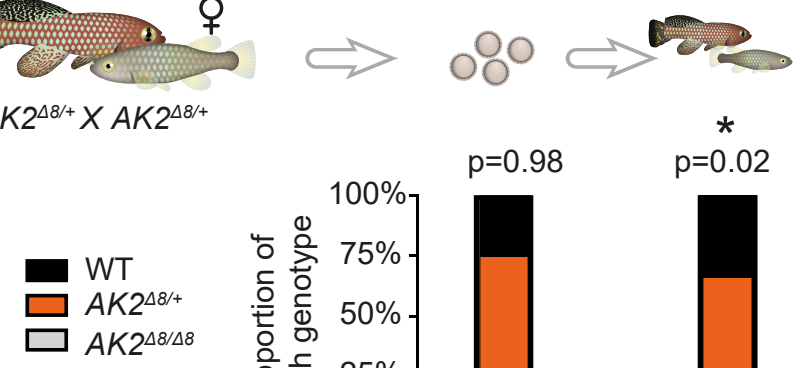

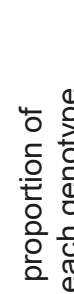

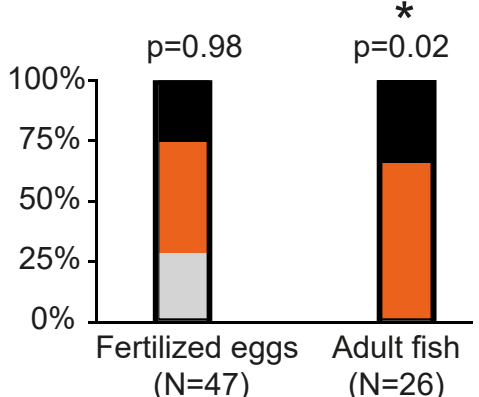

G. $A K 2^{\Delta 8 /+}$ male lifespan

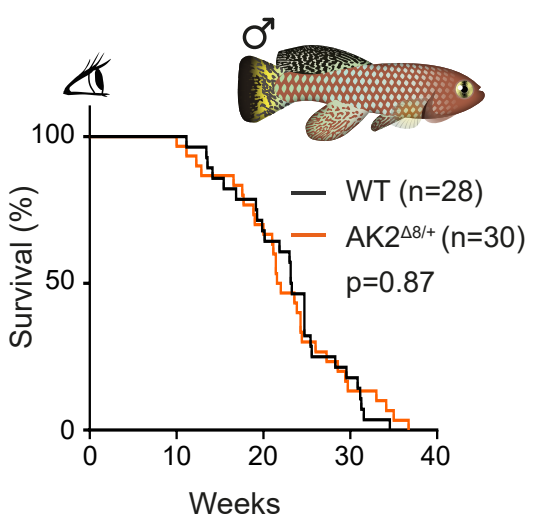

H. $A K 2^{\Delta 8 /+}$ female lifespan

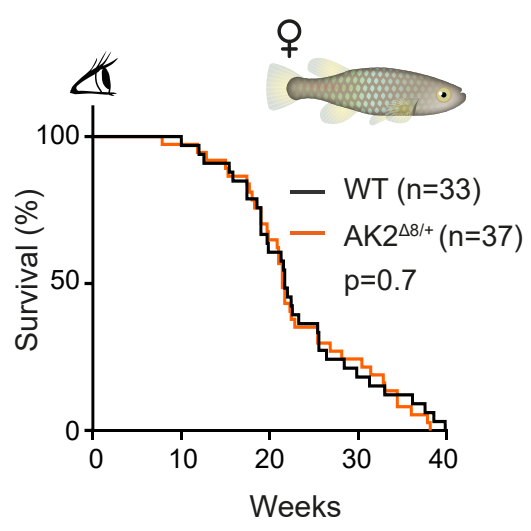




\section{Figure S1: Male and female lifespan is unaffected by the $A K 2^{48 /+}$ heterozygous mutation}

(A) Mating of $A P R T^{\Delta 8 /+}$ heterozygous pairs to generate homozygous $A P R T^{\Delta 8 / \Delta 8}$ mutants. $A P R T^{\Delta 8 / \Delta 8}$ fertilized eggs are observed at the expected Mendelian ratios (no difference between expected and observed frequencies, $\mathrm{p}=0.9, \chi^{2}$ test). APRT ${ }^{\Delta 8 / \Delta 8}$ homozygous adult fish are not detected, indicating embryonic lethality $\left(\mathrm{p}=0.0015, \chi^{2}\right.$ test $)$.

(B) Number of live eggs for each mating pair of the indicated genotypes. Each data point is an average of 4 weekly embryo collections from a single mating pair (a total of 5 pairs per genotype). Bars represent minimum and maximum. Significance was calculated using an unpaired t-test.

(C) Egg size from mating pairs of the indicated genotypes (size is measured as egg diameter, in millimeter). Total number of eggs per experimental condition is presented. Bars represent mean \pm SEM (due to low variation, bars are very small). Significance was calculated using an unpaired t-test.

(D) A phylogenetic tree for adenylate kinase 2 (AK2) protein sequence for classical genetic model systems. A neighbor-joining tree with no distance corrections (Clustal Omega, EBI).

(E) Generation of $A K 2$ CRISPR mutant, including guide RNA (gRNA) target design for exon 2 (red sequence), knock-in of a NheI restriction site (green), and successful germline transmission of an $8 \mathrm{bp}$ deletion $(\Delta 8)$. The $A K 2 \Delta 8$ allele is predicted to generate a protein with a premature stop codon.

(F) Mating of $A K 2^{\Delta 8 /+}$ heterozygous pairs to generate homozygous $A K 2^{48 / \Delta 8}$ mutants. $A K 2^{48 / \Delta 8}$ fertilized eggs are observed at the expected Mendelian ratios (no difference between expected and observed frequencies, $\mathrm{p}=0.98, \chi^{2}$ test). $A K 2^{48 / \Delta 8}$ homozygous adult fish are not detected, indicating embryonic lethality $\left(\mathrm{p}=0.02, \chi^{2}\right.$ test $)$.

$(\mathbf{G}-\mathbf{H})$ Lifespan of WT and $A K 2^{\Delta 8 /+}$ fish performed separately for males $(\mathbf{E})$ and females $(\mathbf{F}) \mathrm{p}$ values for differential survival in log-rank tests, and fish numbers are indicated. See Table S1 for complete statistics. 
Figure S2bioRxiv preprint doi: https://doi.org/10.1101/2022.01.10.475524; this version posted January 10, 2022. The copyright holder for this preprint (which was not certified by peer review) is the author/funder. All rights reserved. No reuse allowed without permission.

A. PCA for transcript levels under different feeding conditions

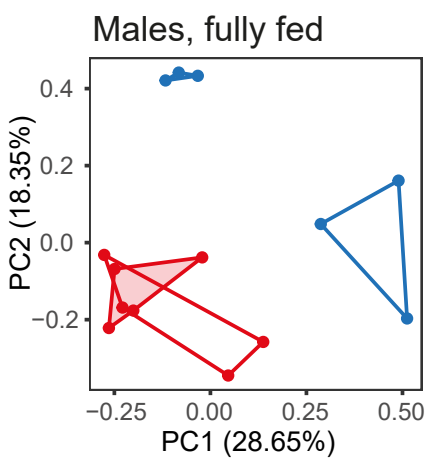

Young WT

$\square$ Old WT

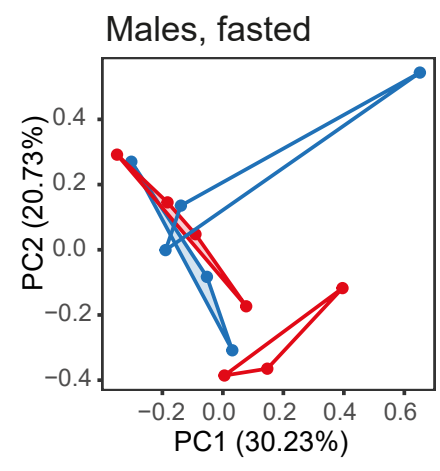

Young APRT $\triangle 8 /+$

$\square$ Old $A P R T^{\Delta 8 /+}$
B. APRT transcript levels are reduced in $A P R T^{\triangle 8 /+}$ male livers

C. Inflammatory processes are enriched during male aging

Regulation of innate immune response
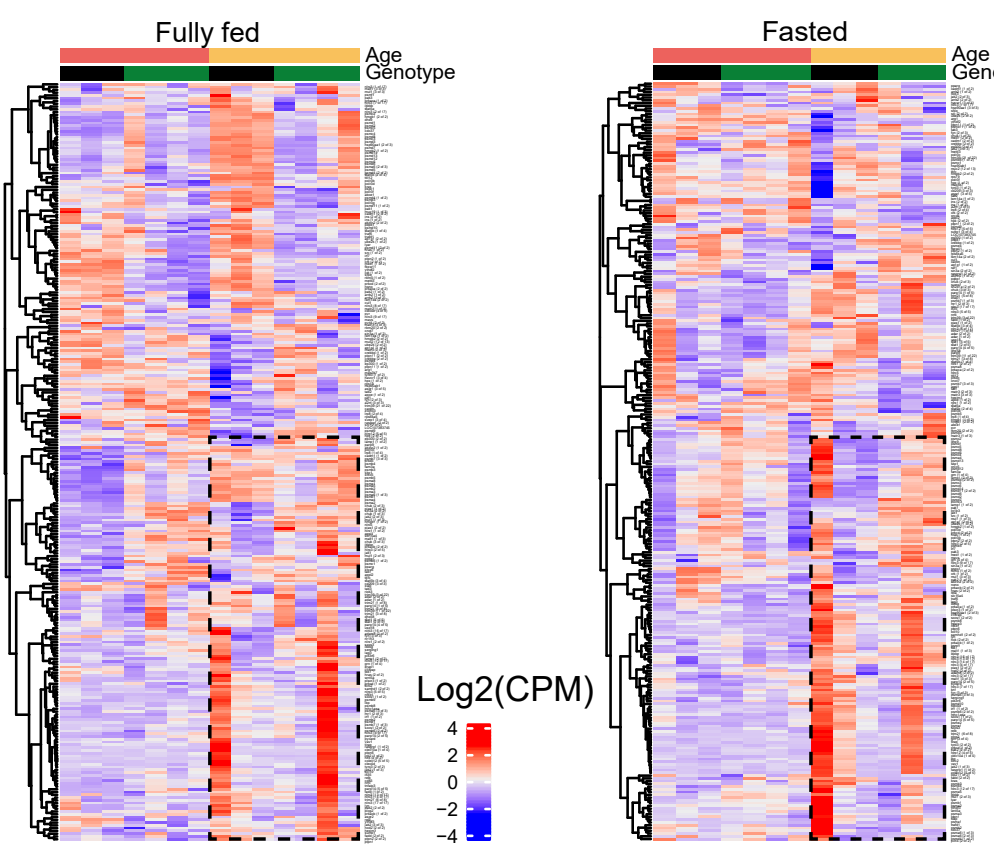

Age

Young

Old

Genotype

a WT

$A P R T^{\triangle 8 /+}$

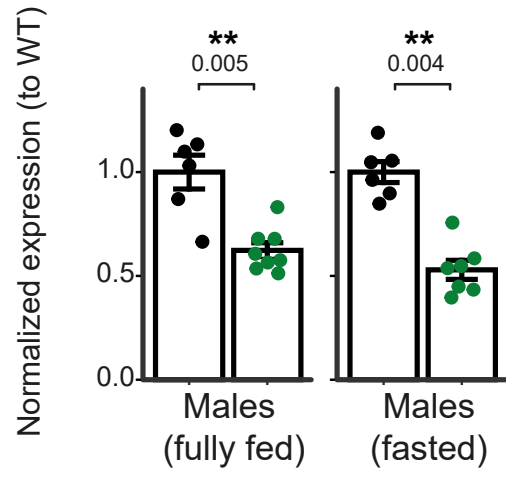

WT

$\square A P R T^{\triangle 8 /+}$

Log2(CPM)

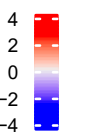




\section{Figure S2: Transcriptomic analysis for male fish}

(A) Principal component analysis (PCA, for PC1 and PC2) for transcript levels from the livers of WT (blue) or $A P R T^{\Delta 8 /+}$ (red) fish, young (shaded) or old (nonshaded). Experiments were performed under fully-fed (top) or fasting conditions (bottom). Each symbol represents an individual fish.

(B) Relative expression of $A P R T$ mRNA in WT and $A P R T^{48 /+}$ fish using RNA-seq. For each condition, expression level is normalized to WT. Each symbol represents an individual fish. Experimental groups differ by genotype and feeding condition (old and young fish are grouped together). Significance was calculated using an unpaired t-test, with FDR correction.

(C) Heatmap for inflammation-related gene expression in male livers (GO term: regulation of innate immune response). Genes are hierarchically clustered, and expression values are presented as z-score on $\log 2(\mathrm{CPM})$. Each column represents an individual fish at the indicated experimental conditions. Dashed lines highlight differences between young and old fish. 
Figure S3bioRxiv preprint doi: https://doi.org/10.1101/2022.01.10.475524; this version posted January 10, 2022. The copyright holder for this preprint (which was not certified by peer review) is the author/funder. All rights reserved. No reuse allowed without permission.

A. Complex age-food-genotype metabolic signature for old $A P R T^{\Delta 8 /+}$ fish

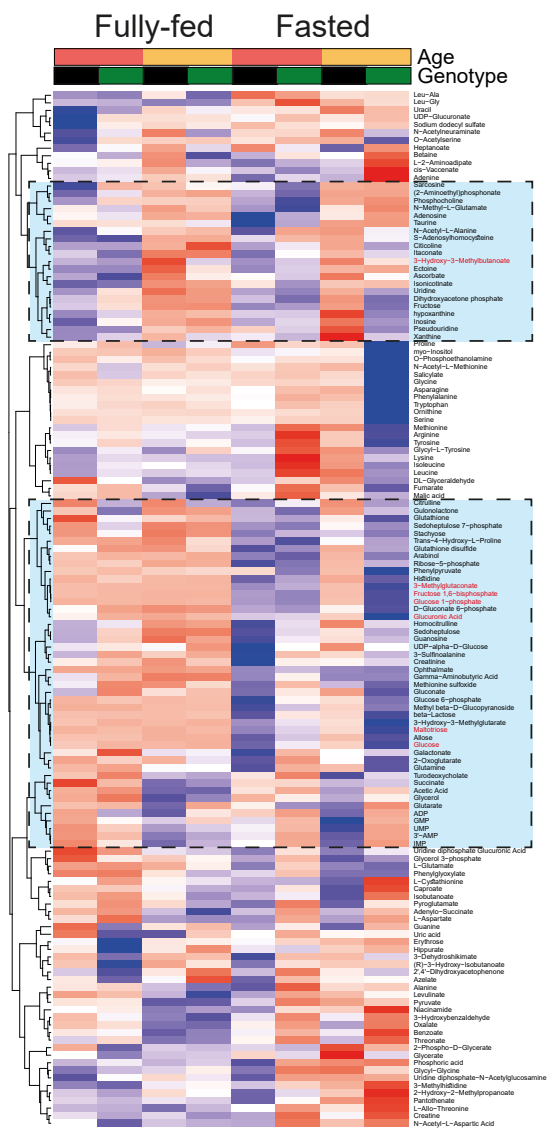

B. PCA for lipid levels under different feeding conditions

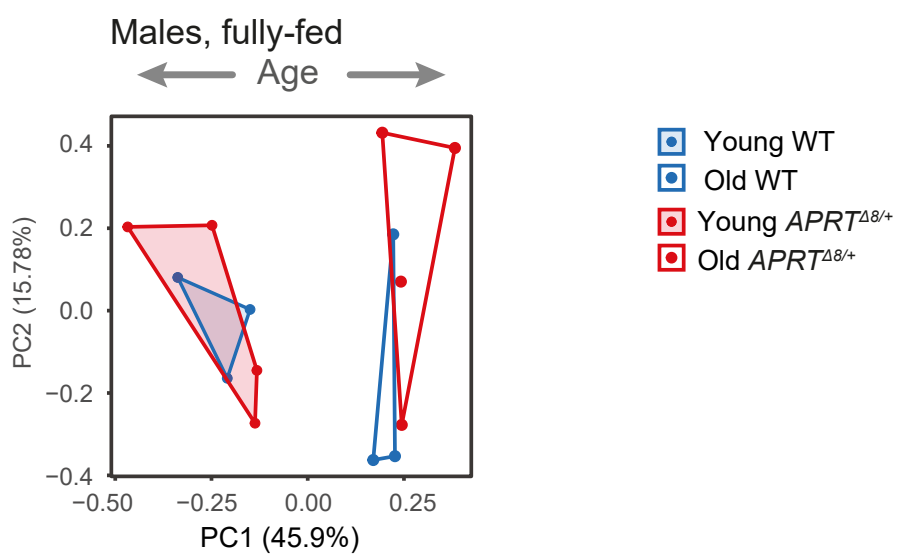

Males, fasted

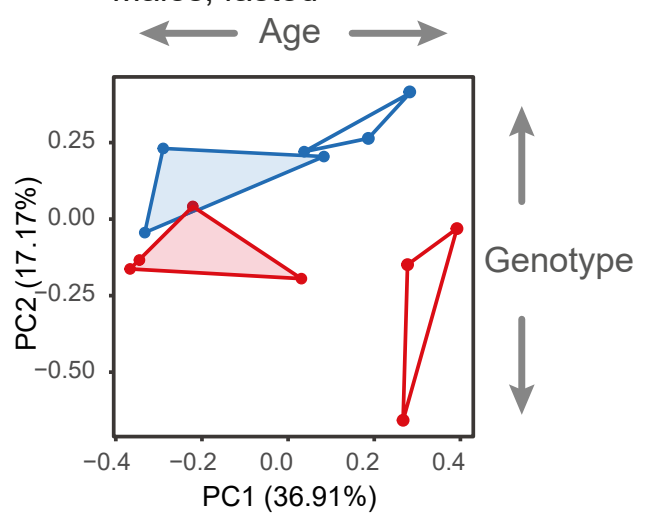

C. Old $A P R T^{\triangle 8 /+}$ fish display a rejuvenated signature for specific lipid species in response to fasting

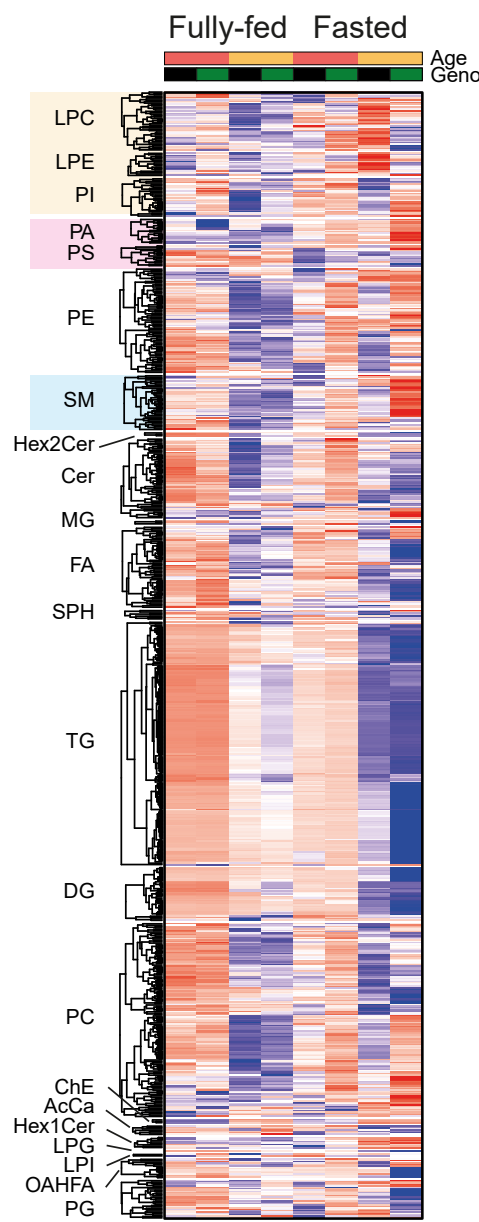

Age
SM

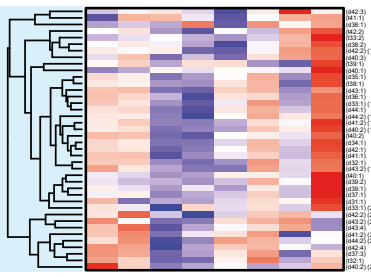

Genotype

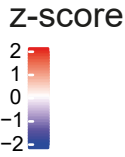

Age Young
Old

Genotype

- WT

APRT ${ }^{\triangle 8 /+}$

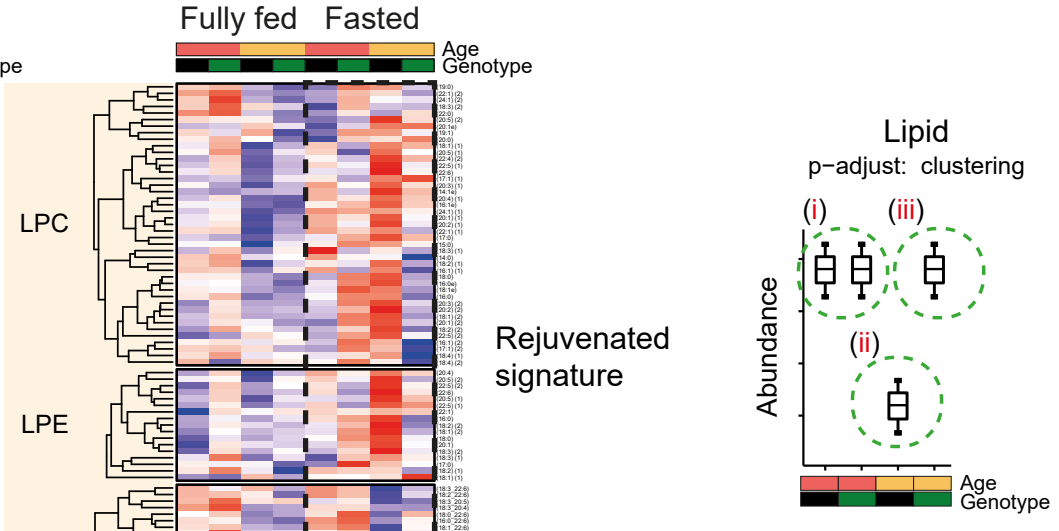

$\operatorname{LPE}(16: 0)$

ANOVA: 0.045

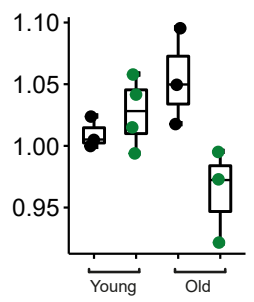

Food
LPC(18:2) (1)

ANOVA: 0.043

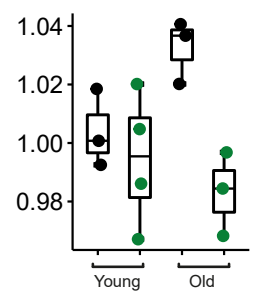

$\mathrm{PI}$ (17:0_20:4) ANOVA: 0.027441

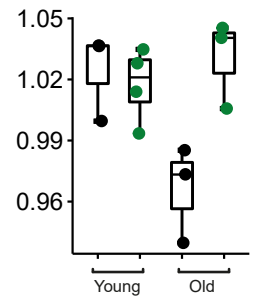

Age \&

Genotype

Age

Young

Old

Genotype

z-score 


\section{Figure S3: Liver metabolomic and lipidomic analysis by age, genotype, and feeding condition in male fish}

(A) Hierarchical clustering of all identified liver metabolites. Each square represents normalized relative abundance (indicated by color) from an average of 3-4 fish of the indicated experimental condition. Each condition differs by age (young or old), genotype (WT or $A P R T^{\Delta 8 /+}$ ), and feeding condition (fully-fed or fasted for $24 \mathrm{~h}$ ). Significant metabolites are in red, and a broader list of selected metabolites that cluster with them are highlighted in blue. Statistical tests as described in Figure 3b. Selected metabolites (blue) were further analyzed in Figure $3 b, 3 c$.

(B) Principal component analysis (PCA) for abundance of lipid species from the livers of WT (blue) or $A P R T^{\Delta 8 /+}$ (red) fish, young (shaded) or old (nonshaded). Experiments were performed under fully-fed (top) or fasting conditions (bottom). Each symbol represents an individual fish. Full metabolite list is reported in Table S3.

(C) Heatmap showing all identified (left) and selected (right) lipid species from the livers of the indicated experimental groups. Specific glycerophospholipids (i.e. PI, LPE, and LPC) display a restoration of youthful levels in old $A P R T^{\triangle 8 /+}$ fish under fasting conditions (right). Other lipid species display a complex dependency on diet (i.e. PA, PS), or age and genotype (i.e. SM). Each square represents normalized relative abundance (indicated by color), from an average of 3-4 fish.

LPC: Lysophosphatidylcholine; LPE: lysophosphatidylethanolamine; PI: Phosphtatidylinositols; PA: Phosphtatidic acids; PS: Phosphtatidylserines; PE: Phosphtatidylethanolamines; SM: Sphingomyelins; Hex1Cer/Hex2Cer: hexa-ceramides 1/2 glycan chains; Cer: Ceramides/Sphingoid bases; MG: Mono(acyl|alkyl) glycerols; FA: Fatty acids; SPH: Ceramides/Sphingoid bases; TG: Tri(acyl|alkyl) glycerols; DG: Di(acyl|alkyl) glycerols; PC: Phosphtatidylcholines; ChE: Cholestrol ester; AcCa: acylcarnitine; LPG: Lysophosphatidylglycerol; LPI: Lysophosphatidylinositol; OAHFA: (OAcyl)- $\omega$-hydroxy fatty acids; PG: Phosphtatidylglycerols. Full lipid list is reported in Table S3.

(D) Boxplot showing trending specific lipid species selected from $(\mathbf{C})$. Each dot represents one fish. Bars represent minimum and maximum. Significant lipids could not be detected due to sample noise. Therefore, our statistical test was limited to fasting conditions. We applied oneway ANOVA between 3 clusters: young (i); old WT (ii); and old $A P R T^{\Delta 8 /+}$ (iii). Using these clusters, we estimated age-dependent deregulated nutrient sensing (comparing clusters $\mathbf{i}$ and $\mathbf{i i}$ ) and rejuvenation in old $A P R T^{\Delta 8 /+}$ fish (comparing clusters ii and iii). Significance was called at FDR $<5 \%$ for ANOVA, and top 5\% for Tukey post-hoc (between groups ii and iii). 
Figure S4ioRxiv preprint doi: https://doi.org/10.1101/2022.01.10.475524; this version posted January 10, 2022. The copyright holder for this preprint (which was not certified by peer review) is the author/funder. All rights reserved. No reuse allowed without permission.

A. PCA for transcript levels in females
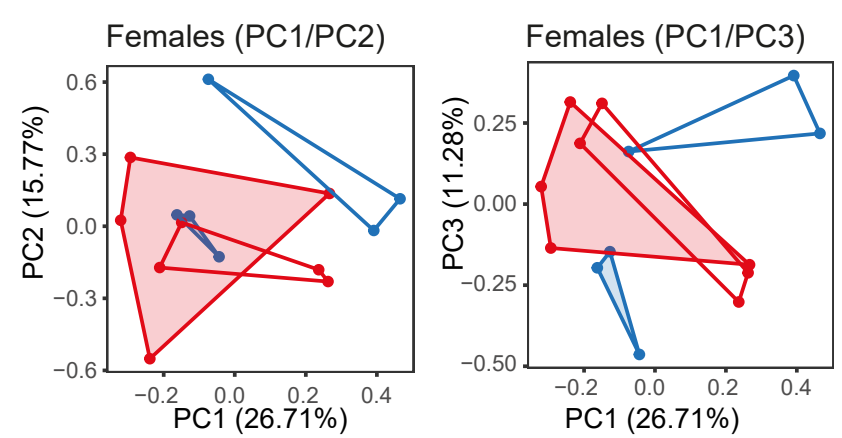

B. APRT transcript levels are reduced in $A P R T^{\triangle 8 /+}$ female livers
$\square$ Young WT
$\square$ Old WT
Young APRT $\triangle 8 /+$
Old $A P R T^{\triangle 8 /+}$

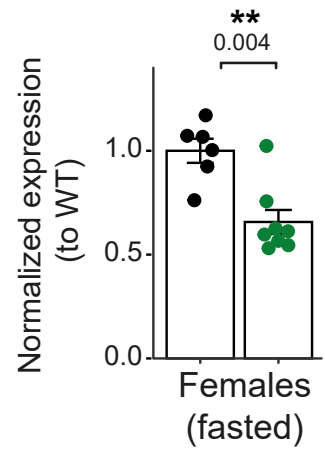

D. Core regulators of mitochondrial dynamics highlight role for $P G C-1 \alpha$
Macroautophagy Organelle fission

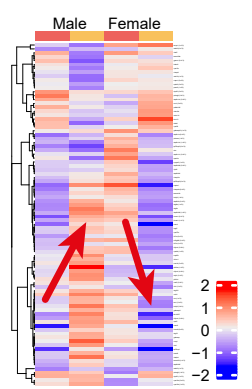

Response to starvation

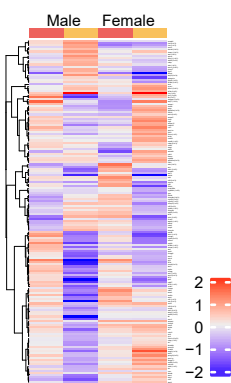

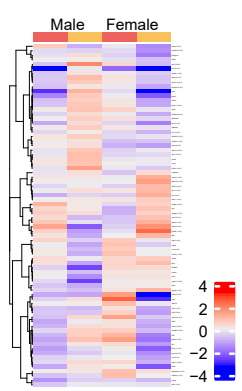

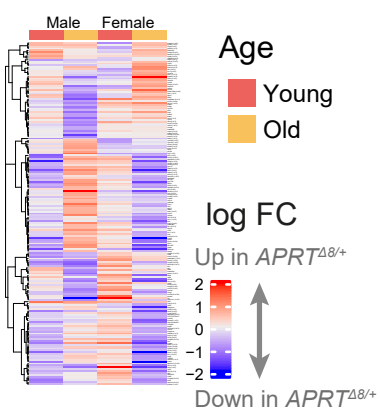

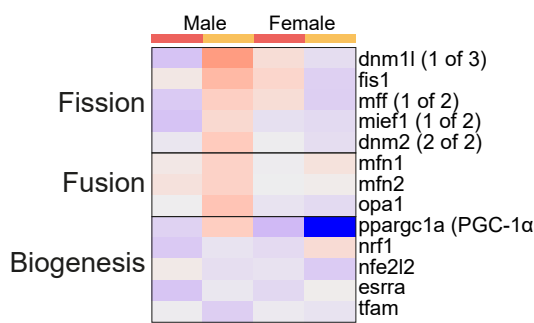

Age

Young

Old $\log$ FC

Up in $A P R T^{\Delta 8 / 4}$

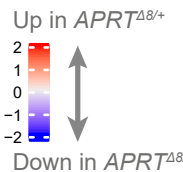




\section{Figure S4: Transcriptomic analysis for female fish}

(A) Principal component analysis (PCA) for transcript levels from the livers of WT (blue) or $A P R T^{\Delta 8 /+}$ (red) female fish, young (shaded) or old (nonshaded). Experiments were performed under fasted conditions. Each symbol represents an individual fish. PC1/PC2 and PC1/PC3 are shown.

(B) Relative expression of APRT mRNA in WT and $A P R T^{\Delta 8 /+}$ fish using RNA-seq. For each condition, expression level is normalized to WT. Each symbol represents an individual fish. Experimental groups differ by genotype (old and young fish are grouped together). Significance was calculated using an unpaired t-test, with FDR correction.

(C) Heatmap of RNA-Seq (log2 fold change) between $A P R T^{\Delta 8 /+}$ and WT expression values using a cutoff of 1.5-fold change. Experimental groups differ by sex (male or female) and age (young or old). Selected pathways are from Figure 4d, and red arrows highlight age-related changes in gene expression.

(D) Heatmap of RNA-Seq (log2 fold change) between $A P R T^{\Delta 8 /+}$ and WT expression values depicting core regulators of mitochondrial fusion, fission, and biogenesis. Experimental groups differ by sex (male or female) and age (young or old). 
Figure S5 bioRxiv preprint doi: https://doi.org/10.1101/2022.01.10.475524; this version posted January 10, 2022. The copyright holder for this preprint (which was not certified by peer review) is the author/funder. All rights reserved. No reuse allowed without permission.

A. Overlapping Genotype-Age interactions identify sex-specific enrichments for ncRNA processing
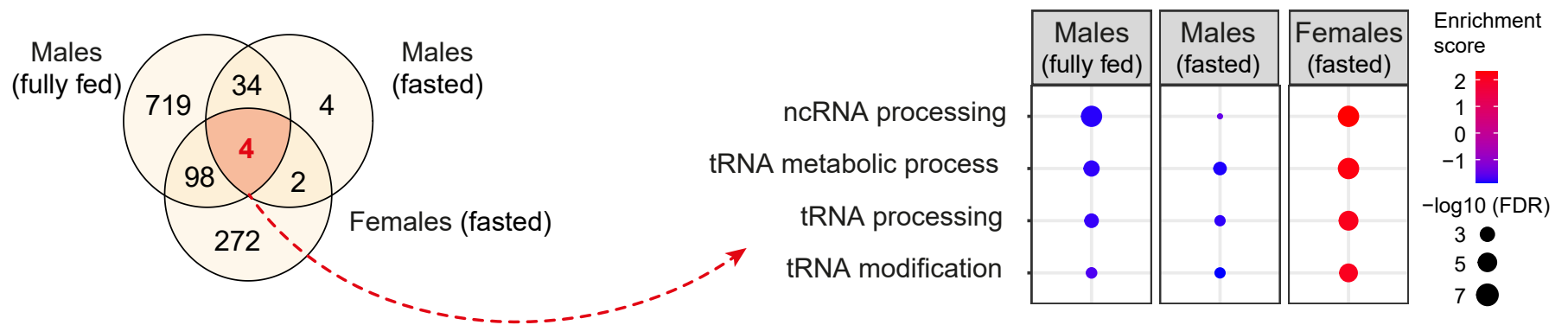

B. Hyper RNA-editing occurs primarily in non-coding regions

ADAR motifs at different genomic regions
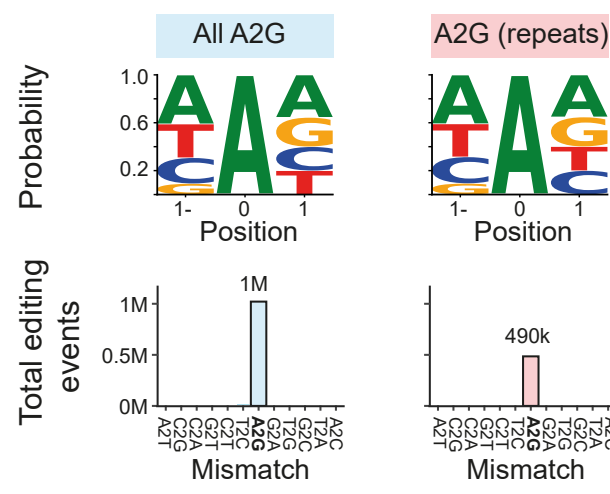

A2G (CDS)
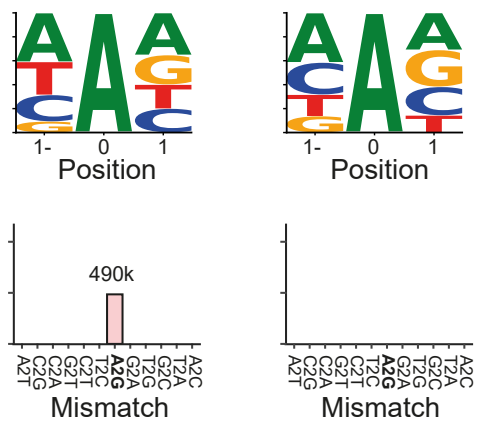

C. RNA-editing is sex- and age-dependent within hyper edited regions

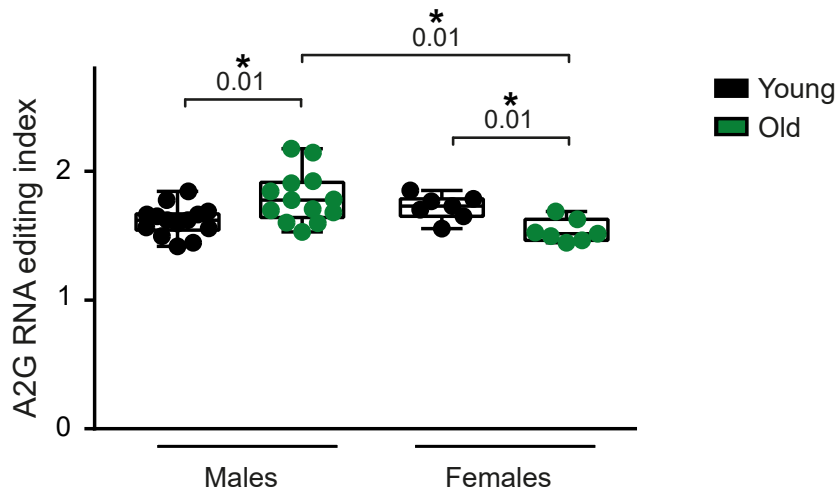

D. Within hyper edited regions, RNA-editing is not dependent on age, genotype, feeding, or sex
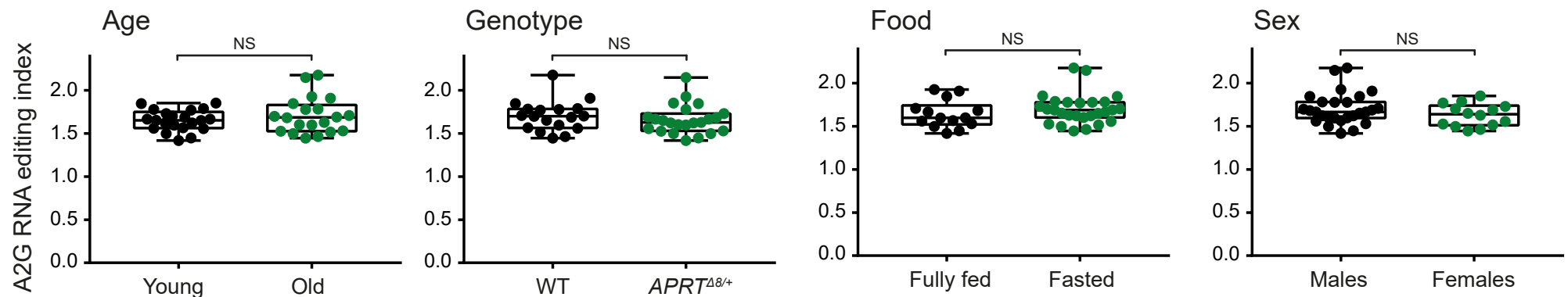


\section{Figure S5: RNA-editing is sex- and age-dependent}

(A) Venn diagram showing the overlap of significantly enriched pathways that were identified by Genotype-Age interaction analysis across all experimental groups (left). Analysis was performed separately for each experimental condition (i.e. male fully-fed, male fasted, and female fasted). GO enrichments using GSEA were called at FDR $<5 \%$ (right).

(B) Identification of A-to-I hyper editing in killifish at different genomic regions across all experimental groups. Signal-to-noise was assessed by estimating the nucleotide frequencies at the hyper edited sites (that matched the ADAR deamination motif, top), and by the number Ato-G mismatches compared to all types of mismatches (bottom).

(C) Stratifying RNA-editing by experimental conditions highlights significant sex- and agedependency within hyper edited regions. Additional comparisons are available in Figure S5c. Each symbol represents an individual fish. Bars represent minimum and maximum, and significance was measured by multiple independent unpaired t-test with FDR correction.

(D) Stratifying RNA-editing by experimental conditions. Each symbol represents an individual fish. Bars represent minimum and maximum. Significance was measured by an independent unpaired t-test, and exact p-values are indicated in table S4. 
Figure S6ioRxiv preprint doi: https://doi.org/10.1101/2022.01.10.475524; this version posted January 10, 2022. The copyright holder for this preprint (which was not certified by peer review) is the author/funder. All rights reserved. No reuse allowed without permission.

A. Glycolytic function profile ('ECAR assay')

B. Altered glycolytic function in primary cells from wild-type and $A P R T^{\Delta 8 /+}$ fish ('ECAR assay')
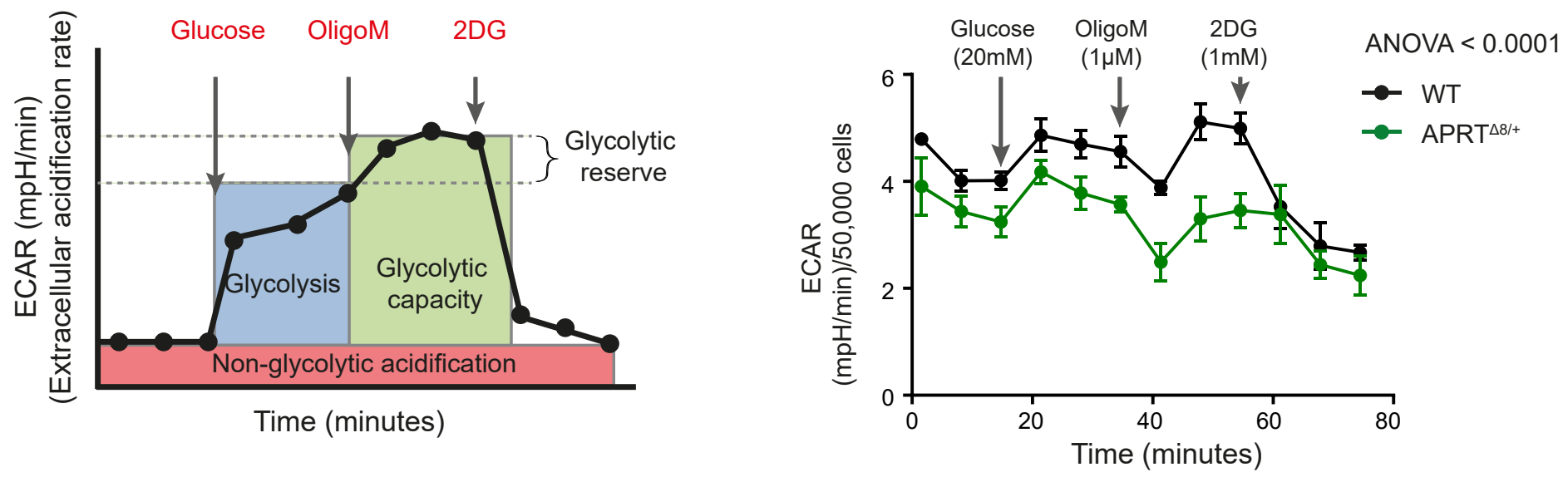

C. Reduced specific glycolytic functions in $A P R T^{\triangle 8 /+}$ ('ECAR assay')

Glycolytic capacity

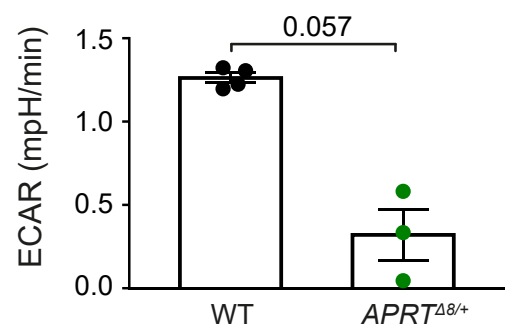

Glycolytic reserve

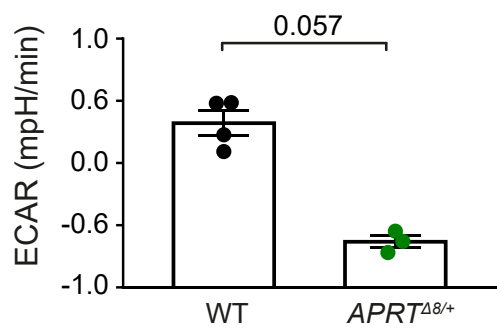

Glycolysis $\quad \rightarrow$ WT

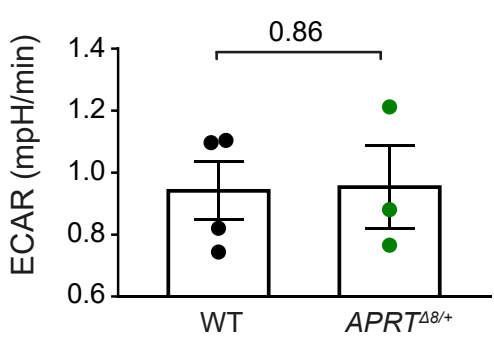

D. Mitochondrial ATP production is unaltered in $A K 2^{\Delta 8 /+}$-derived cells

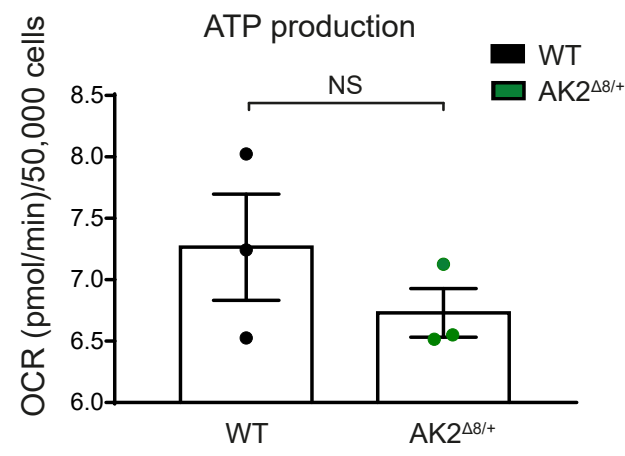

F. Reduced mitochondrial respiration in $A P R T^{\Delta 8 /+}$-derived female cells

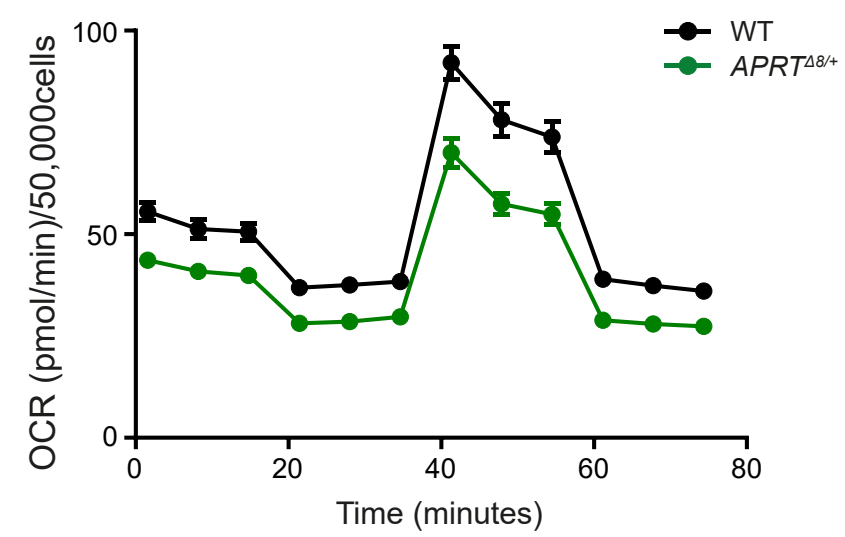

E. Mitochondrial DNA copy number in primary cells derived from either male or female fish
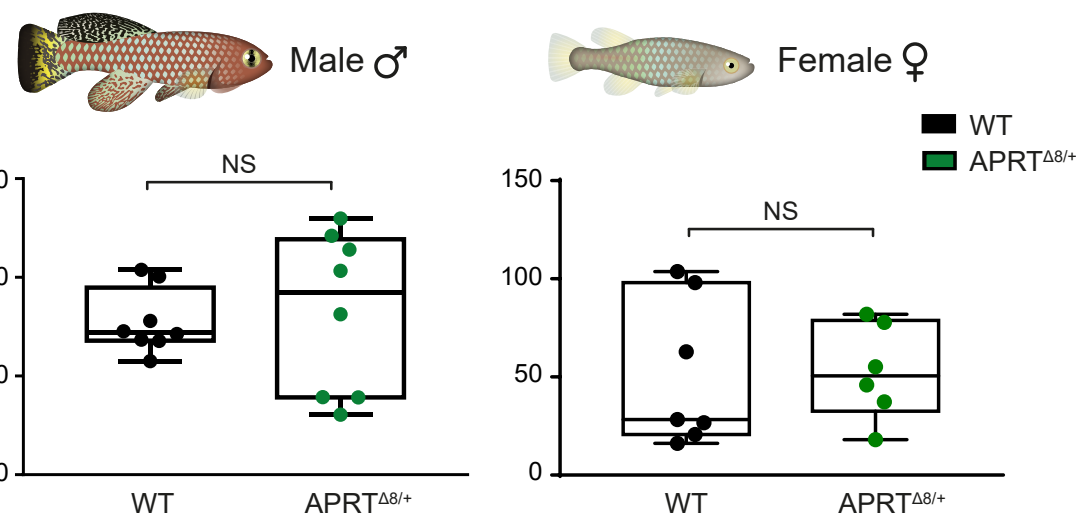

G. Estradiol (E2) does not significantly affect mtDNA copy number in fish liver
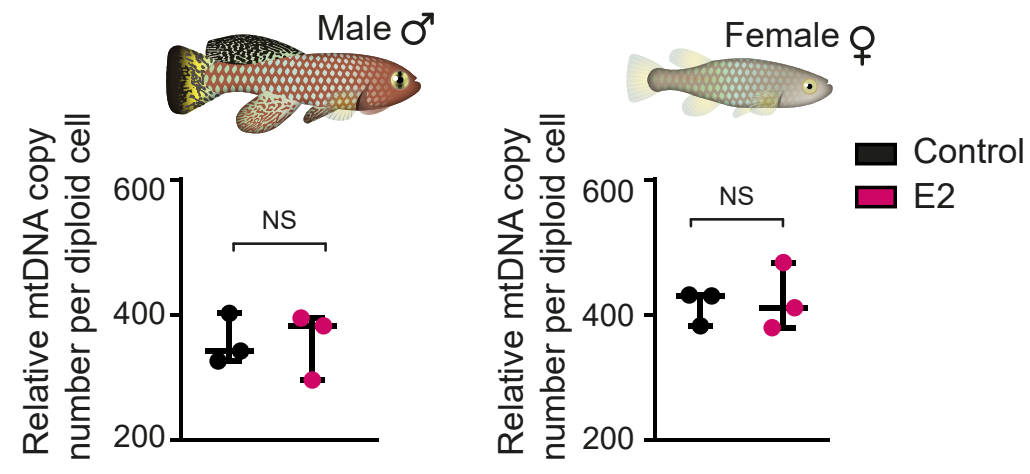


\section{Figure S6: Characterization of mitochondrial functions and copy number}

(A) Measurement of glycolytic function profile by extracellular acidification rate (ECAR assay, $\mathrm{mpH} / \mathrm{min}$ ) using the Seahorse Glycolysis Stress Test Kit (Agilent). Measurements were performed under basal conditions, or following the addition of glucose, oligomycin (OligoM, an ATP synthase inhibitor), or 2-deoxy glucose (2-DG, a glucose analog, that inhibits glycolysis). Specific parameters were further normalization by actual cell numbers. $n=3-4$ for each genotype.

(B) Measuring glycolytic function in WT- and $A P R T^{\Delta 8 /+}$-derived primary cells. Each symbol represents an average of three independent measurements using cells derived from 3-4 individual fish. Error bars represent mean \pm SEM, and significance was measured using twoway ANOVA and BKY correction.

(C) Glycolytic capacity (left), Glycolytic reserve (center), and Glycolysis (right) were compared between WT- and $A P R T^{\Delta 8 /+}$-derived primary cells. Each symbol represents an individual fish. Error bars represent mean \pm SEM, significance was measured using MannWhitney test, and exact p-values are indicated.

(D) ATP production was compared between WT- and $A K 2^{48 /+}$-derived primary cells. Each symbol represents an individual fish. Error bars represent mean \pm SEM, and significance was measured using Mann-Whitney test.

(E) Relative mtDNA copy number was determined by quantitative real-time PCR (qPCR) using primers for mitochondrial (16S rRNA) and nuclear (Cdkn2a/b) gene. Each symbol represents a primary culture isolated from an individual male fish of the indicated genotype. Bars represent minimum and maximum and significance was estimated by unpaired t test.

(F) Measuring mitochondrial respiration in WT- and $A P R T^{\Delta 8 /+}$-derived primary cells, isolated from female fish. Each symbol represents an average of three independent measurements using cells derived from three individual fish. Error bars represent mean \pm SEM, and significance was measured by two-way ANOVA and BKY correction.

(G) Relative mtDNA copy number in adult fish livers was determined by qPCR using primers for mitochondrial (16S rRNA) and nuclear $(C d k n 2 a / b)$ gene. Males (left) and females (right) were fed either control food (black), or food supplemented with E2 (pink). Each symbol represents an individual fish. Bars represent minimum and maximum, and significance was measured using Mann-Whitney test . 
Fiqure S70ioRxiv preprint doi: https://doi.org/10.1101/2022.01.10.475524; this version posted January 10, 2022. The copyright holder for this preprint (which was not certified by peer review) is the author/funder. All rights reserved. No reuse allowed without permission.

A. Conservation of antibody antigens

\begin{tabular}{|c|c|c|c|}
\hline Antibody & Antigen alignme & nt (UniProt) & Size \\
\hline AMPKa CST-5831 & $\begin{array}{l}\text { Q13131|AAPK1_HUMAN } \\
\text { A0A1A8B983|A0A1A8B983_NOTFU }\end{array}$ & $\begin{array}{ll}\text { AEKQKHDGRVKIGHYIL } 29 \\
\text { TEKQKHEGRVKIGHYIL } 19 \\
: \star \star \star \star \star \star * \star * \star \star \star \star \star \star \star \star *\end{array}$ & kDa 62 \\
\hline $\begin{array}{r}\text { pAMPKa CST-2535 } \\
(\text { Thr172) }\end{array}$ & $\begin{array}{l}\text { P54646 |AAPK2_HUMAN } \\
\text { A0A1A8B983|A0A1A8B983_NOTFU }\end{array}$ & 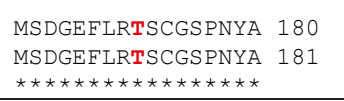 & kDa 62 \\
\hline ACC CST-3676 & $\begin{array}{l}\text { Q13085|ACACA_HUMAN } \\
\text { A0A1A8A9C5|A0A1A8A9C5_NOTFU }\end{array}$ & 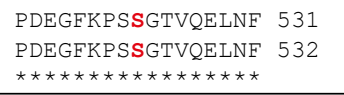 & kDa 280 \\
\hline pACC CST-3661 & $\begin{array}{l}\text { P11497|ACACA_RAT } \\
\text { A0A1A8A9C5|A0A1A8A9C5_NOTFU }\end{array}$ & $\begin{array}{c}\text { AFHMRSSMSGLHLVKQG } 87 \\
\text { INNLRPSMSGLHLVKQG } 89 \\
:: * \star \star \star \star \star \star \star \star \star \star \star *\end{array}$ & kDa 280 \\
\hline RPS6 CST-2217 & $\begin{array}{l}\text { P62753|RS6_HUMAN } \\
\text { A0A1A8UHL8|A0A1A8UHL8_NOTFU }\end{array}$ & $\begin{array}{l}\text { HKRRRIALKKQRTKKNK } 203 \\
\text { HKRRRIALKKQRTQKNK } 203 \\
\star \star \star \star \star \star \star \star \star \star \star \star \star \star * * \star *\end{array}$ & kDa 32 \\
\hline pRPS6 CST-2211 & $\begin{array}{l}\text { P62753|RS6_HUMAN } \\
\text { A0A1A8UHL8|A0A1A8UHL8_NOTFU }\end{array}$ & 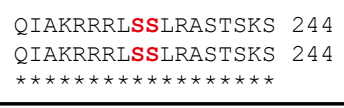 & kDa 32 \\
\hline
\end{tabular}

B. Antibody validation

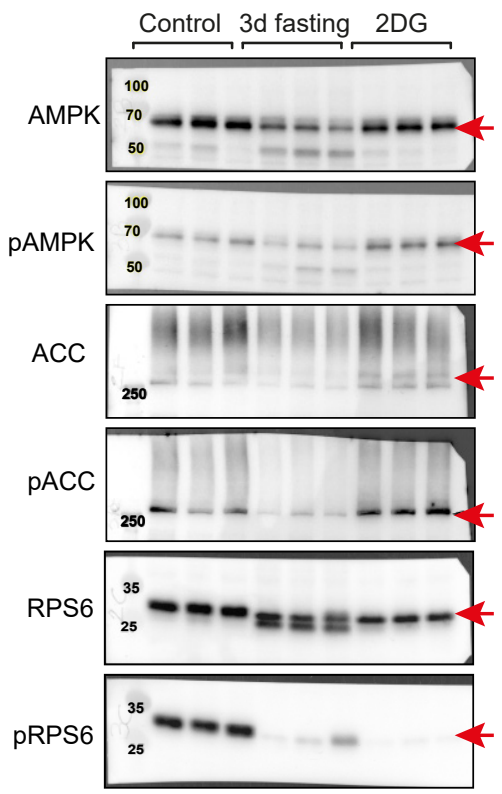

C. Fasting reduces pRPS6 levels

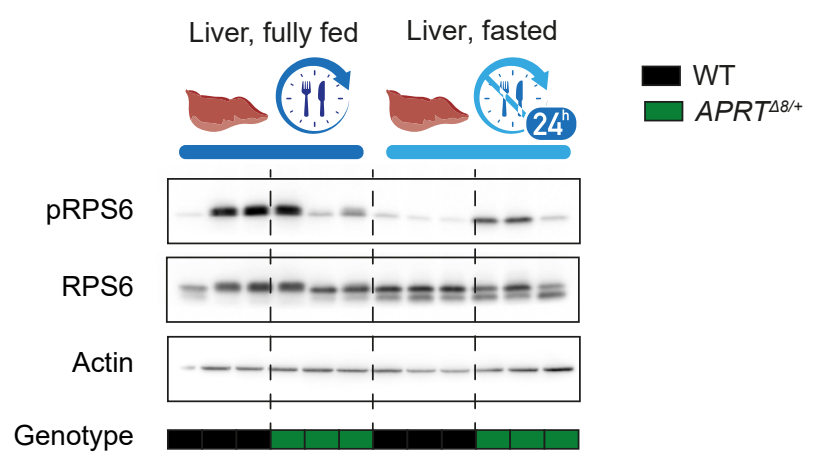

E. Maturation of male $A P R T^{\Delta 8 /+}$ fish

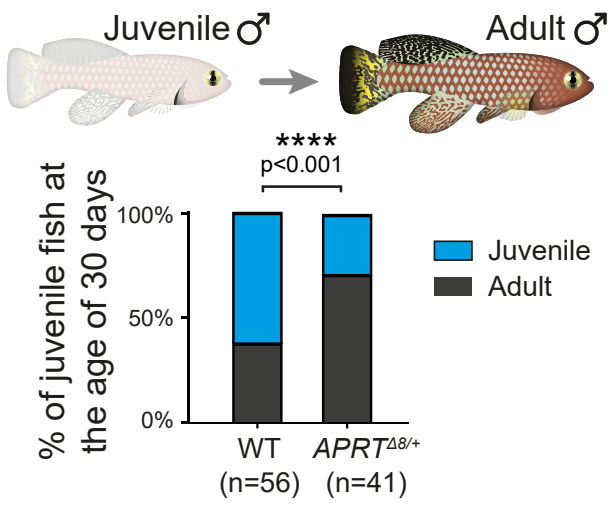

D. Growth of $A P R T^{\triangle 8 /+}$ male and female fish
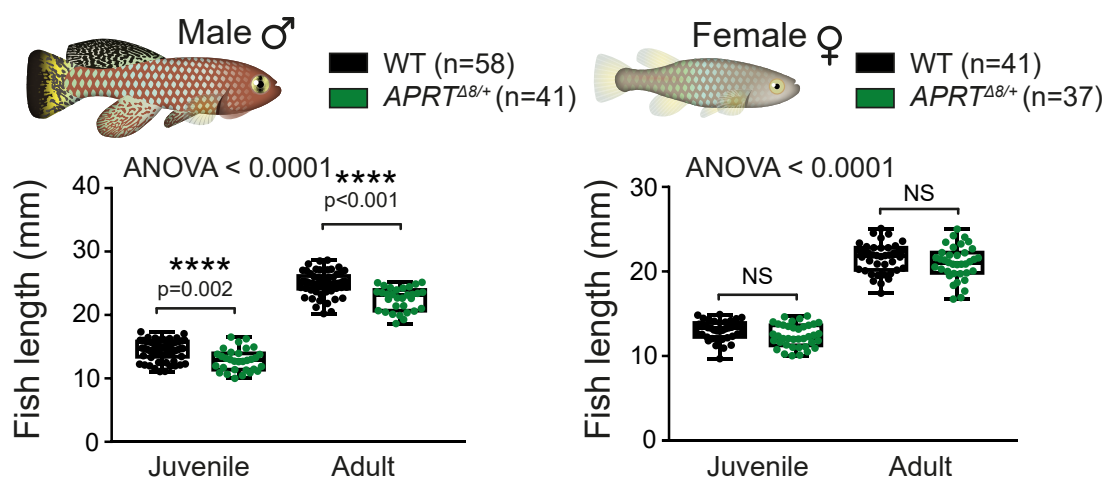

F. High-fat diet increases lipid droplets in old wild-type male fish, but not in young

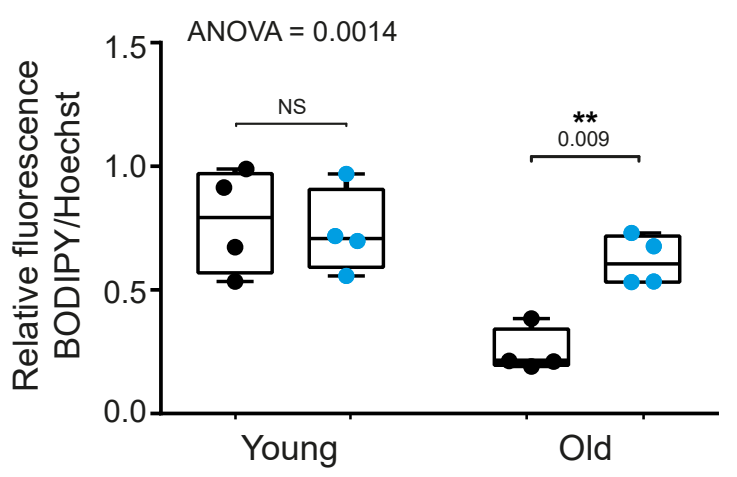

Fully fed

High-fat diet 


\section{Figure S7: The effect of the APRT mutation on metabolic and physiological parameters}

(A) Protein sequence conservation of the antigen sequence of the indicated antibodies and the corresponding killifish protein.

(B) Validation of key antibodies used in this manuscript. Western blot of livers isolated from adult male fish under i) control condition; ii) chronic AMPK pathway activation via prolonged fasting ( $72 \mathrm{~h}$ fasting); iii) or acute AMPK pathway activation via immersion in 2 deoxy glucose (2DG, $20 \mathrm{mM}$ for $3 \mathrm{~h}$ ). Data are from 3 biological replicates for each experimental condition.

(C) Western blot of livers isolated from old WT and $A P R T^{48 /+}$ male fish. Experiments were performed under either control or $24 \mathrm{~h}$ fasting conditions. Data are from 3 biological replicates for each experimental condition.

(D) Measurement of growth rates during fish maturity. Experimental groups differ by sex (males or females), age (14 dph juveniles or $30 \mathrm{dph}$ adults), and genotype (WT or $A P R T^{\Delta 8 /+}$ ). Number of replicates for each experimental group are indicated (the same animals were measures as juveniles and adults). Bars represent minimum and maximum, and significance was measured by one-way ANOVA and Sidak's multiple comparisons test. Exact p-values are indicated.

(E) Scoring the onset of male maturity (using color changes as a proxy) in WT or APRT ${ }^{48 /+}$ fish at different ages (juvenile or adults). Number of replicates for each experimental group are indicated. Significance was measured by Fisher's exact test, and exact p-values are indicated.

(F) Hepatic lipid accumulation and quantification in response to high-fat diet. Quantification of the relative fluorescence intensity of BODIPY/Hoechst (Fiji-ImageJ). Experimental groups differ by age (young or old) and feeding condition (control or high-fat diet). Bars represent minimum and maximum, and significance was measured by one-way ANOVA and Sidak's multiple comparisons test. Exact p-values are indicated. 